\title{
Experimental Investigation of Scattering from Randomly Rough Elastic Cylinders
}

by

\author{
John Van Gurley
}

B.S., Physics

University of Florida (1986)

Submitted in partial fulfillment of the

requirements for the degrees of

OCEAN ENGINEER

and

MASTER OF SCIENCE IN OCEAN ENGINEERING

at the

MASSACHUSETTS INSTITUTE OF TECHNOLOGY

and the

WOODS HOLE OCEANOGRAPHIC INSTITUTION

September 1992

\begin{tabular}{c} 
MARINE \\
BIOLOGICAL \\
LABORATORY \\
\hline LIB RARY \\
\hline $\begin{array}{c}\text { WOOOS HOLE, MASS. } \\
\text { W. H. O. I. }\end{array}$ \\
\hline
\end{tabular}

(C) John Van Gurley, 1992

The author hereby grants to MIT, WHOI and the U.S. Government permission to reproduce and to distribute copies of this thesis document in whole or in part.

Signature of Author

Joint Program in Applied Ocean Science and Engineering Massachusetts Institute of Technology Woods Hole Oceanographic Institution August 7, 1992

Certified by

Dr. Timothy K. Stanton Associate Scientist

Woods Hole Oceanographic Institution Thesis Supervisor

Accepted by

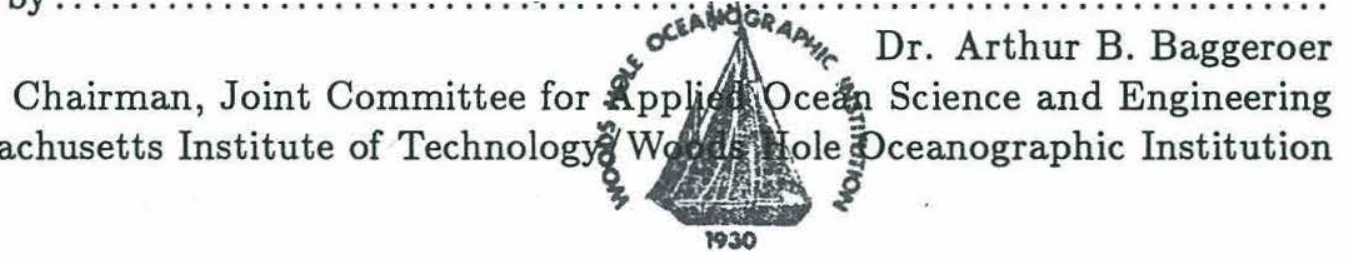




\title{
Experimental Investigation of Scattering from Randomly \\ Rough Elastic Cylinders
}

by

John Van Gurley

\author{
Submitted to the Massachusetts Institute of Technology/ \\ Woods Hole Oceanographic Institution \\ Joint Program in Applied Ocean Science and Engineering \\ on August 7, 1992, in partial fulfillment of the \\ requirements for the degrees of \\ Ocean Engineer \\ and \\ Master of Science in Ocean Engineering
}

\begin{abstract}
Acoustical backscattering from randomly rough infinitely long elastic cylinders surrounded by a fluid medium is examined. The cylinder radius is allowed to vary along its lengthwise axis creating one-dimensional rotationally symmetric roughness. Using recently published rough cylinder formulations [T.K. Stanton, J. Acoust. Soc. Am., 92, 1641-1664 (1992) and T.K. Stanton and D. Chu, J. Acoust. Soc. Am., 92, 1665-1678 (1992)], explicit expressions are derived for the backscattered field for a laboratory pulse-echo environment: spherically spreading directional source and receiver with arbitrary beam patterns. Efficient numerical integration algorithms are developed to solve for the backscattered field from a specified surface profile. Experimental measurements from dense elastic (stainless steel) cylinders immersed in water are presented to quantitatively illustrate the effects of small scale surface roughness $\left(\sigma_{s} / a=0.0131\right.$ where $\sigma_{s}$ is the surface rms roughness and $a$ is the mean cylinder radius) for $4.5<k a<70$ where $k$ is the acoustic wavenumber. The actual target surface profile is well described and used as an input in the numerical simulations. Agreement is found between measurements and simulation predictions both in the mean field levels and the field fluctuations over a wide range of frequencies.
\end{abstract}

Thesis Supervisor: Dr. Timothy K. Stanton

Associate Scientist

Woods Hole Oceanographic Institution 


\section{Acknowledgements}

The last two years have been extremely rewarding and enjoyable due, in large part, to the many friends and colleagues who have provided help and encouragement along the way. Although I cannot recognize everyone, I would like to thank those who have been instrumental in the success of this research.

First and foremost, a special thanks my thesis advisor, Tim Stanton. His guidance and enthusiasm were central to the completion of this work. While allowing me much latitude in my approach, he ensured that I never lost my way.

I am deeply indebted to Rob Fricke and Craig Schultz for their help in completing the experimental measurements. Their divine intervention rescued me from utter dispair. I wish to express my thanks to Nafi Toksoz for generously making available the laboratory facilities at ERL. I am also grateful to the many others who supplied assistance and moral support most notably Tom Austin, Paul Boutin, Dezhang Chu, Matt Conti, Chick Corrado, Kevin LaPage, and especially Matt Johnson.

I will be forever grateful to Joe Bondaryk, John Buck, and Bob Eastwood who collectively held my hand as I walked through the valley of UNIX. They were patient when I was simply ignorant and sympathetic when the computer gods refused to cooperate.

I would like to thank Chris Howell for the daily updates from the Nation's Newspaper and my many official and unofficial officemates who helped maintain a stimulating, if not always productive, environment in 5-435.

I wish to express my sincere thanks to the U.S. Navy and the Oceanographer of the Navy for giving me this opportunity. Their generous support made my participation in the Joint Program possible.

Finally, I am deeply grateful to my wife, Kim, and son, Kyle, whose love and patience have seen me through the many long hours of work, cheering me up when things were not going well, and leaving the room when things REALLY were not going well. This work is dedicated to them. 


\section{Contents}

$\begin{array}{ll}\text { List of Figures } & 6\end{array}$

1 Introduction $\quad 12$

2 Theoretical Development $\quad 15$

2.1 General solutions . . . . . . . . . . . . . . . . . 15

2.1.1 Basic equations . . . . . . . . . . . . . . 15

2.1.2 Exact modal series solution for undeformed infinitely long

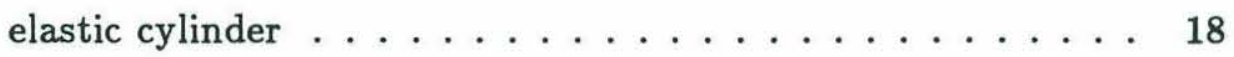

2.1.3 Simplified ray solution for infinitely long elastic cylinder . . . 24

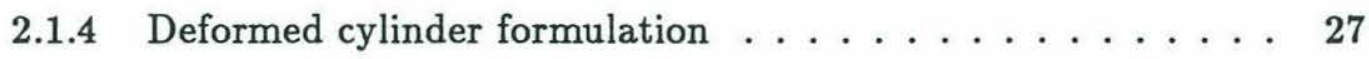

2.1.5 Modification for point source and receiver with arbitrary directivity patterns . . . . . . . . . . 30

2.1.6 Extension to randomly rough cylinders . . . . . . . . . 31

2.2 Explicit solution for rough infinitely long cylinders $\ldots \ldots \ldots . \ldots 32$

2.3 Fluctuations in the scattered field . . . . . . . . . . . 35

3 Numerical Analysis $\quad 39$

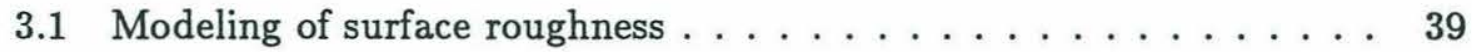

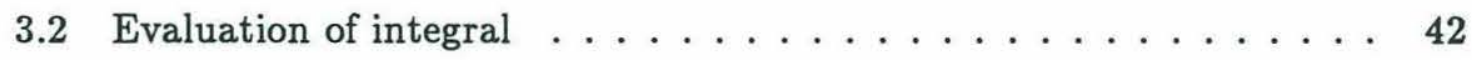

3.3 Comparison of smooth and rough cylinder solutions $\ldots \ldots \ldots 4$ 
4 Experimental Measurements

4.1 Target design and manufacture ............... 56

4.2 Experimental set-up and alignment . . . . . . . . . . . . . . 64

4.3 Data acquisition and signal processing . . . . . . . . . 67

4.4 Calibration of system . . . . . . . . . . . 71

4.5 Experimental data and data selection ........... 77

4.6 Comparison with numerical results . . . . . . . . . . . 90

5 Discussion of Results

104

6 Conclusions

109

Bibliography

111 


\section{List of Figures}

2-1 General scattering geometry for an infinitely long cylinder. . . . . . 20

2-2 "Ray" diagram implied by Sommerfeld-Watson transformation showing the specular, $m=0$ Rayleigh and Franz wave contributions to backscattering from elastic cylinders. . . . . . . . . . . .

2-3 Scattering geometry for a plane wave incident upon a deformed finite cylinder with a point receiver. From Stanton [16]. . . . . . . . .

2-4 Scattering geometry for deformed cylinder and general bistatic geometry (directional transmitter and receiver). . . . . . . . . .

2-5 Geometry for (monostatic) backscatter from a rough infinitely long cylinder aligned along $z$ axis. The mean radius $a_{o}$ and perturbation, $\varsigma(z)$, component of the surface profile $a_{R C}(z)$ are shown. . . . . . 32

2-6 Phasor diagram showing mean and fluctuation components of echo. . 36

2-7 Rice PDF of scattering amplitude for various values of $\gamma$. For $\gamma=0$ the curve is the Rayleigh PDF and for $\gamma \gg 1$ the Rice PDF approaches a Gaussian. PDF $\equiv w_{R}(f)\left\langle|f|^{2}\right\rangle^{1 / 2} \ldots \ldots \ldots \ldots \ldots$

3-1 Histogram for rough surface simulation showing Gaussian nature of the profile statistics. 100 random sinusoids were summed to provide the profile. The input rms roughness $\sigma_{s}$ was $100 \mu \mathrm{m}$. The area under the histogram has been normalized to 1 to give a perturbation PDF. The superimposed Gaussian PDF was calculated with the same standard deviation $\sigma_{s} \ldots \ldots \ldots \ldots \ldots \ldots \ldots$

3-2 Converged modal summation for $0 \leq k a \leq 30$. The summation quickly converges for $m \gtrsim k a$ so the limits of summation were changed from $\sum_{m=0}^{\infty}$ to $\sum_{m=0}^{k a+10}$ for efficient calculation. . . . . . . . . . . .

3-3 Real part of integrand for infinite cylinder. (a) and (b) represent typical smooth cylinder integrands while (c) and (d) correspond to rough cylinder $\left(\sigma_{s}=60 \mu \mathrm{m}\right)$ integrands for the same values of $k a$. The value of $k a(k a=11.3)$ in (a) and (c) is in a slowly varying region of the modal summation, away from any nulls. In (b) and (d), $k a=12.75$ is just above a sharp null in the modal term. The imaginary part of the integrand shows the same structure. . . . . . 
3-4 Magnitude of the backscattered form function $\left(|f| \equiv\left|f^{\infty}\right|\right.$ from Eq. 2.81) from a smooth infinite-length elastic cylinder for a spherically spreading directional source and receiver with a Gaussian beam pattern. This numeric solution is based on the approximate deformed cylinder formulation. Input variables are sound speed for fresh water $c=1477 \mathrm{~m} / \mathrm{s}$, and the material properties for stainless steel $c_{s}=3100, c_{l}=5790 \mathrm{~m} / \mathrm{s}$, and $\rho_{1}=7.9$. The source distance and beamwidth were $R=24 \mathrm{~cm}$ and $\Theta_{o}=26^{\circ}$. Rapid oscillations at $k a \approx$ $6,12,16,19$, etc. are due to the incomplete cancellation of higher order Fresnel zones near sharp modal nulls due to the spherically spreading incident field. . . . . . . . . . . . . .

3-5 Backscattering from randomly rough infinite-length elastic cylinders for a spherically spreading directional source and receiver with a Gaussian beam pattern and fractional roughness $\sigma_{s} / a=0.005$. The scatter plot superimposes field solutions for 150 simulated realizations. The elastic material properties are the same as Figure 34 and the surface roughness bandlimits are $K_{\text {low }}=169 \mathrm{~m}^{-1}$ and $K_{\text {high }}=2934 \mathrm{~m}^{-1}$. As $k a$ increases, variability is shown to increase indicating increased effects due to roughness. . . . . . . . . . . .

3-6 Backscattering from randomly rough infinite-length elastic cylinders with fractional roughness $\sigma_{s} / a=0.0131$. See Figure 3-5 for details. .

3-7 Backscattering from randomly rough infinite-length elastic cylinders with fractional roughness $\sigma_{s} / a=0.02$. See Figure 3-5 for details. . . 50

3-8 Backscattering from randomly rough infinite-length elastic cylinders with fractional roughness $\sigma_{s} / a=0.04$. See Figure 3-5 for details. . .

3-9 Mean backscattered field from randomly rough infinite-length elastic cylinders for various values of fractional roughness $\sigma_{s} / a$. Solutions for mean field calculated from an ensemble average of 300 simulated realizations. (a) is mean of data in Fig. 3-5, (b) Fig. 3-6, (c) Fig. 3-7, and (d) Fig. 3-8. . . . . . . . . . . . . . . 51

3-10 (a) Variance $\left(\sigma_{|f \infty|}^{2}\right)$ and (b) relative variance $\left(\sigma_{|f \infty|}^{2} /\left\langle\left|f^{\infty}\right|^{2}\right\rangle\right)$ for backscattered field from randomly rough cylinders. Numerical simulation based on 300 realizations for $\sigma_{s} / a=0.0131$. . . . . . . . . . .

3-11 Histograms of normalized scattering amplitude $\left|f^{\infty}\right| /\left\langle\left|f^{\infty}\right|^{2}\right\rangle^{1 / 2}$ from 300 simulated realizations (Fig. 3-10) for various $k a$ values. Rice PDFs are superimposed with shape parameter $\gamma$ calculated from the fluctuations of $\left|f^{\infty}\right| . \ldots \ldots \ldots \ldots . . \ldots \ldots$

3-12 Rice PDF shape parameter $\gamma$ plotted against $k a$ and $k \sigma_{s} .300$ numerical realizations per $k a$ were used to calculate $\gamma$. The figure illustrates the rapid decrease in $\gamma$ as $k$ increases and the transition to saturation at $k \sigma_{s} \approx 0.5 \ldots \ldots \ldots \ldots \ldots \ldots$ 
4-1 Cutaway diagram showing target and threaded pin joining different subsections. Each rough subsection is $\approx 15 \mathrm{~cm}$ long and has a mean diameter $0.9126 \mathrm{~cm}$. Threaded pins $0.47625 \mathrm{~cm}$ in diameter and 1.27 cm long were used to screw the pieces together. . . . . . . .

4-2 Six rough cylinder subsections comprising randomly rough target shown to scale. . . . . . . . . . . . . . . . .

4-3 Histogram of radius for each of six rough surface subsections (a) (f) and entire composite target profile (g). A Gaussian PDF has been superimposed on each plot highlighting the Gaussian nature of the profile statistics. . . . . . . . . . . . . . .

4-4 MEM estimate of the surface power density spectrum. The spectrum shows the good approximation between the actual surface and a bandlimited white process. The design band edges are $\mathcal{K}_{\text {low }}=811 \mathrm{~m}^{-1}$ and $K_{\text {high }}=2565 \mathrm{~m}^{-1} \ldots \ldots \ldots \ldots$

4-5 Autocorrelation $\left(\Phi_{z z}\right)$ function for the target profile (only center 20

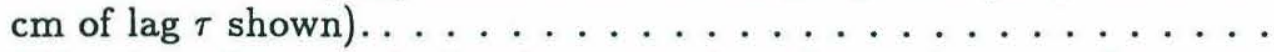

4-6 Side view of test tank showing mounting of transducers and targets approximating a monostatic backscattering measurement. . . . . . .

4-7 Transducer and target configurations for data measurements: (a) for beam pattern measurements, and (b) for rough cylinder backscatter measurements. ......................... 67

4-8 Laboratory setup for experiments. . . . . . . . . . . . . . 68

4-9 Schematic diagram of data acquisition system and motor controllers. 70

4-10 Modified Hanning window used in data acquisition: (a) close-up of leading edge (and trailing edge) of 1024 point window for time series showing smoothing due to the applied 40 point Hanning edge.(b) Fourier transform of modified Hanning window vs rectangular window. . . . . . . . . . . . . . . . . 72

4-11 Waterfall plot of time series from beam pattern measurement. . . . . 73

4-12 Greyscale plot of Fourier transformed beam pattern time series showing frequency response vs. frequency and azimuth angle $(\Phi)$ for transducer from $-40^{\circ} \leq \Phi \leq 40^{\circ}\left(\Phi=0^{\circ}\right.$ is "broadside"). . . . . . . . .

4-13 Greyscale plot of signal to noise ratio (SNR) for beam pattern measurements vs frequency and $\Phi$. . . . . . . . . . . . . . 75

4-14 Frequency response measurements for near center beam $\left(\Phi \leq \pm 6^{\circ}\right) . \quad 76$

4-15 Transducer frequency response averaged over frequency to give "average" beam pattern vs $\Phi$. The response was averaged over the bandwidth from $1 \mathrm{MHz}$ to $3 \mathrm{MHz}$. A fitted Gaussian (- - ) with characteristic beamwidth $\Theta_{0}=26^{\circ}$ is superimposed for comparison.

4-16 Configuration of rough cylinder subsections for data runs 1 through 3.78 
4-17 Time series for smooth cylinder alignment and calibration measurements taken throughout the experimental run. Position 1 was directly over the joint between the two $20 \mathrm{~cm}$ smooth cylinder subsections. The transducers were moved $2 \mathrm{~cm}$ along the $z^{\prime}$ axis toward the end of the target between positions. . . . . . . . . .

4-18 Tank echo measurements using exactly the same alignment and transducer motions as Figure 4-17 except with the target removed from the mounts. This shows some return off of the mounts at the extremum position 4 . The returns are roughly $40 \mathrm{~dB}$ below that from the cylinder. . . . . . . . . . . . .

4-19 Time series for rough cylinder measurements in run 1 . The transducer mount was moved $2 \mathrm{~cm}$ along the $z^{\prime}$ axis between measurements and the target configuration is shown in Figure 4-16. A typical smooth cylinder time series is shown to compare the Rayleigh wave arrival times. . . . . . . . . . . . . .

4-20 Time series for rough cylinder measurements in run 2. A typical smooth cylinder time series is shown to compare the Rayleigh wave arrival times. . . . . . . . . . . . . .

4-21 Time series for rough cylinder measurements in run 3. A smooth cylinder time series is shown to compare the Rayleigh wave arrival times. . . . . . . . . . . . . . . . .

4-22 Sample SNR levels for (a) smooth cylinder and (b) rough cylinder measurements. ..................

4-23 Calibration curves obtained by inverting the smooth cylinder "standard" frequency response from Figure 4-17 (measurement 3-2) and the numerical solution for elastic cylinder scattering . (a) Raw curve showing fine structure due to mismatch between nulls. (b) Curve after low-pass filtering using a fourth order Butterworth filter to smooth the data. . . . . . . . . . . . . . .

4-24 Comparison of inverted calibration curve (-) and measured response from beam pattern measurements at different azimuth angles(- - -). .

4-25 Normalized smooth cylinder frequency response measurements. Note missing nulls at $k a=5.0,8.0$, and 10.0 in positions $3-1,4-1,5-1$, and $6-1$ corresponding to measurements taken at a joint between subsections. . . . . . . . . . . . . . .

4-26 Normalized rough cylinder frequency response measurements for runs

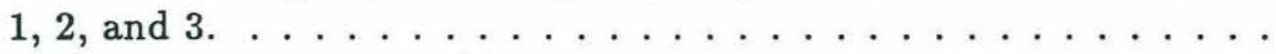

4-27 Measured backscattered (.) field from a smooth "infinite"-length stainless steel cylinder vs predicted field (-) from numerical simulation. Scatter plot formed from measurements taken at 12 different positions along the cylinder's $z$ axis. . . . . . . . . . 
4-28 Mean of measured backscattered field from smooth "infinite"-length cylinder $\left({ }^{*}\right)$ vs predicted field (-). Mean measured field is an ensemble average of the 12 measurements in Figure 4-27. Errorbars indicate \pm 1 standard deviation, $\sigma_{\left|f^{\infty}\right|}$, from the 12 measurements at each frequency.

4-29 (a) Variance $\sigma_{|f \infty|}^{2}$ and (b) relative variance $\sigma_{|f \infty|}^{2} /\left\langle\left|f^{\infty}\right|\right\rangle^{2}$ of smooth cylinder backscatter data over the 12 realizations.

4-30 Sample SNR level (-) for smooth cylinder measurements overlaid with the relative variance $(\cdot)$ from Figure $4-29(b) . \ldots \ldots \ldots \ldots$

4-31 Measured backscattered field $(\cdot)$ from a randomly rough "infinite"length stainless steel cylinder vs predicted minimum and maximum from Monte Carlo simulations with additive noise (-) and without additive noise (- - -). Scatter plot formed from measurements of 44 realizations along the target axis and the numerical simulation is based on 50 simulated realizations using the profile of the target. Additive Gaussian white noise has been included in the Monte Carlo simulations to model the system related noise observed in the smooth cylinder data. . . . . . . . . . . . . . . . .

4-32 Relative variance $\sigma_{|f \infty|}^{2} /\left\langle\left|f^{\infty}\right|^{2}\right\rangle$ of rough cylinder backscatter data over the 44 realizations $\left({ }^{*}\right)$ vs numerical simulation including additive Gaussian white noise $(-) \ldots \ldots \ldots \ldots \ldots$

4-33 Sample SNR level (-) for rough cylinder measurements overlaid with the relative variance from Figure $4-32(\ldots) \ldots \ldots \ldots \ldots$

4-34 Relative variance $\sigma_{|f \infty|}^{2} /\left\langle\left|f^{\infty}\right|\right\rangle^{2}$ of rough cylinder backscatter data $\left({ }^{*}\right)$ vs the numerical prediction (-) after smoothing using a 21 point weighted running average. Simulation includes additive Gaussian white noise. . . . . . . . . . . . . . .

4-35 Histogram of measured rough cylinder backscatter data for various $k a$ values overlaid with Rice PDF using measured $\gamma(---)$. These $k a$ values were chosen away from modal nulls. $k \sigma_{s}=0.0733,0.117$, $0.235,0.278,0.440,0.533$ for plots $(\mathrm{a})-$ (f) respectively. . . . . . .
Histogram of measured rough cylinder backscatter data for various $k a$ values overlaid with Rice PDF using measured $\gamma(---)$. These $k a$ values were chosen atmodal nulls. . . . . . . . . . . . . . . . . 101

4-37 (a) Rice PDF shape parameter, $\gamma$, vs $k a$ and $k \sigma_{s}$. Rough cylinder backscatter data $(*)$ is compared with an ideal or noiseless numeric simulation of 50 realizations (- - -) and numeric simulation with additive Gaussian white noise (-).(b) Same data after smoothing using 11 point weighted running average. The standard deviation of the additive noise was matched to the observed standard deviation "floor" in the smooth cylinder data $\left(\sigma_{|f \infty|}=0.05\right) \ldots \ldots \ldots \ldots$ 
4-38 Mean of measured backscattered field from a randomly rough "infinite"length cylinder $\left(^{*}\right)$ vs predicted mean field (--) overlaid with measured smooth cylinder mean field (o) and smooth cylinder solution $(\cdots)$ from Fig. 4-28. The rough cylinder measured mean field $\left({ }^{*}\right)$ is an ensemble average of the 44 measurements in Fig. 4-31. . . . . . . 103 


\section{Chapter 1}

\section{Introduction}

Fundamental knowledge of basic scattering processes has long provided the foundation for a variety of remote sensing systems: sonars (or "echo sounders") and bioacoustic ultrasonic medical imagers in the acoustic realm, radar and optical lasar systems in the electromagnetic realm. Accurate understanding of the interaction between an incident field and a target of interest is imperative in order to extract any information from the scattered field of an unknown target or, conversely, to accurately predict the scattered field from a known object. Historically, scattering models have been based on analytic solutions for simple mathematical shapes such as spheres, infinitely long cylinders, spheroids, or planar interfaces. But as remote sensing systems have improved and moved from detection and localization to identification and imaging, these simple scattering models fail to provide an accurate picture of scattering from complex naturally occurring objects.

Many investigators have extended the basic models to account for variations in material composition, deformed shapes, and internally loaded structures. However, the perfectly smooth surface boundary condition remains an underlying assumption in the vast majority of this work. Previous research in rough surface scattering has almost exclusively focused on planar boundaries. While two-dimensional rough surface theories have proven crucial for accurate understanding of scattering from 
the ocean surface $[1,2]$, sea ice $[3]$, and the ocean bottom $[2,4]$, little work has focused on the problem of rough three-dimensional bodies. The research conducted has been limited to cases such as simple deformations of spheres $[5,6]$, idealized cylinders (impenetrable bodies insonified by uniform plane waves) $[7,8,9,10]$, or numerical approaches to more complex shapes $[11,12]$.

In addition to the small amount of theoretical attention, few controlled experiments have been conducted to investigate rough surface scattering from bodies of curvature. The published experimental data are typically confined to electromagnetic scattering [13] with only broad similarities to acoustic scattering. Additionally, the surface profiles in these experiments are not known a priori and only estimated, in part, from the scattered field data.

In a series of papers $[14,15,16]$, Stanton recently developed an approximate model for scattering from deformed elongated bodies. Although the original papers only explicitly examined gross deformations about the major axis, such as a curved cylinder, the formulation itself is quite general and has been used to model rotationally symmetric or one-dimensional roughness as a radius deformation down the major axis of the body $[17,18]$. This method can easily describe a variety of shapes and realistic elastic bodies as well as idealized rigid objects.

This thesis will extend Stanton and Chu's rough cylinder formulation $[17,18]$ that involves plane wave incident fields and point receivers to one with realistic acoustic transducers. Specifically, we will incorporate a spherically spreading directional source and receiver with arbitrary beam patterns into Stanton's rough body theory and derive analytic and numerical results for an infinitely long elastic cylinder with small scale roughness. Chapter 2 reviews the normal mode and Sommerfeld-Watson transform (SWT) solutions for the ideal infinitely long elastic cylinder. Stanton's deformed cylinder formulation is presented and extended to directional spherically spreading incident fields. Chapter 3 develops efficient numerical algorithms to solve the rough cylinder formulations and Monte Carlo simulations are presented pre- 
dicting the statistics for the rough cylinder scattered field. Chapter 4 presents data from a number of backscattering experiments using an elastic cylinder target with a known random profile. The experiments measured the backscattered field from a number of statistically independent realizations of the random profile. Since the target profile is specified a priori and well characterized, analytic predictions from the numerical models are compared directly with the experimental results. Chapters 5 and 6 discuss and summarize the results and present the conclusions. 


\section{Chapter 2}

\section{Theoretical Development}

\subsection{General solutions}

\subsubsection{Basic equations}

The general equations for sound propagation in a fluid were first derived in a linear form by Rayleigh [19] over one hundred years ago but still provide the foundation for all work in acoustics. The derivation is based on three basic equations governing the dynamics of motion in an inviscid fluid, here following the form from Morse and Ingard [20] and Skudrzyk [21]. First, the adiabatic equation of state relating the pressure, $p$, and density, $\rho$, of the medium is linearized

$$
p=c^{2} \rho+\text { constant }
$$

where $c$ is the acoustic sound speed in the medium. Next, linearizing the conservation of momentum for a fluid parcel yields Euler's equation

$$
\frac{\partial u_{i}}{\partial t}=\frac{-1}{\rho} \frac{\partial p}{\partial x_{i}}
$$

where $u_{i}$ is the particle velocity in the $x_{i}$ direction and $\rho$ is now the mean density of the medium. Finally, the continuity equation

$$
\frac{\partial u_{i}}{\partial x_{i}}=\frac{-1}{\rho c^{2}} \frac{\partial p}{\partial t}
$$


is a linearized equation for the conservation of mass. Taking a spatial derivative of Eq. (2.2), a time derivative of Eq. (2.3), and subtracting the results yields the standard homogeneous acoustic wave equation

$$
\nabla^{2} p-\frac{1}{c^{2}} \frac{\partial^{2} p}{\partial t^{2}}=0 .
$$

If we consider motions with a harmonic time dependence, $e^{-i \omega t}$, where $\omega(=2 \pi f)$ is the angular frequency, then Eq. (2.2) becomes in, vector form,

$$
\mathbf{u}=\frac{-i}{\omega \rho} \nabla p .
$$

Using the relation $c=\omega / k$ where $k(=2 \pi / \lambda)$ is the acoustic wavenumber and $\lambda$ is the acoustic wavelength, we get the standard relation between the particle velocity and the pressure

$$
\mathbf{u}=\frac{-i}{c k \rho} \nabla p .
$$

Rayleigh [19] and Morse and Ingard [20] derive the scattered field from a infinite circular cylinder by solving the wave equation in a cylindrical coordinate system and matching boundary conditions. Using the cylindrical coordinates $r, \phi, z$ and an $e^{-i \omega t}$ time dependence, Eq. (2.4) becomes

$$
\frac{\partial^{2} p}{\partial r^{2}}+\frac{1}{r} \frac{\partial p}{\partial r}+\frac{1}{r^{2}} \frac{\partial^{2} p}{\partial \phi^{2}}+\frac{\partial^{2} p}{\partial z^{2}}+k^{2} p=0 .
$$

Assuming a separable solution of the form $p=R(r) \Phi(\phi) Z(z) e^{-i \omega t}$, Eq. (2.7) reduces to the coupled equations

$$
\begin{gathered}
\frac{d^{2} Z}{d z^{2}}+k_{z}^{2} Z=0 \\
\frac{d^{2} \Phi}{d \phi^{2}}+m^{2} \Phi=0 \\
\frac{d^{2} R}{d r^{2}}+\frac{1}{r} \frac{d R}{d r}+\left(k_{r}^{2}-\frac{m^{2}}{r^{2}}\right) R=0 \\
\text { where } \quad k_{r}^{2}+k_{z}^{2}=k^{2} \\
k_{r}, k_{z}, m=\text { constants. }
\end{gathered}
$$


The general solution for Eq. (2.8) is

$$
Z=A e^{i k_{x} z}+B e^{-i k_{z} z}
$$

and for Eq. (2.9)

$$
\Phi=A \cos (m \phi) \text { or } A \sin (m \phi)
$$

where $m$ must be an integer for the solution to be periodic in $2 \pi$ as required for axially symmetric problems. The radial equation, Eq. (2.10), is a form of Bessel's equation [22] and has two sets of general solutions

$$
R=\left\{\begin{array}{l}
a J_{m}\left(k_{r} r\right)+b N_{m}\left(k_{r} r\right) \\
\text { or } \\
a H_{m}^{(1)}\left(k_{r} r\right)+b H_{m}^{(2)}\left(k_{r} r\right)
\end{array}\right.
$$

where $J_{m}\left(k_{r} r\right)$ is the Bessel function of the first kind of order $m, N_{m}\left(k_{r} r\right)$ is the Bessel function of the second kind (Neumann function), $H_{m}^{(1)}\left(k_{r} r\right)$ is the Hankel function of the first kind (Bessel function of the third kind) of order $m$, and $H_{m}^{(2)}\left(k_{r} r\right)$ is the Hankel function of the second kind. These solutions are related through the identities

$$
\begin{aligned}
& H_{m}^{(1)}\left(k_{r} r\right) \equiv J_{m}\left(k_{r} r\right)+i N_{m}\left(k_{r} r\right) \\
& H_{m}^{(2)}\left(k_{r} r\right) \equiv J_{m}\left(k_{r} r\right)-i N_{m}\left(k_{r} r\right)
\end{aligned}
$$

Since $N_{m}\left(k_{r} r\right)$ becomes infinite as its argument goes to zero, the solution for any domain containing the origin must be a function of $J_{m}\left(k_{r} r\right)$ only. Finally, using the asymptotic expansion for the Hankel functions at large argument [23]

$$
\begin{aligned}
& H_{m}^{(1)}(x) \underset{k r \gg 1}{\rightarrow} \sqrt{\frac{2}{\pi x}}(-i)^{m} e^{i(x-\pi / 4)} \\
& H_{m}^{(2)}(x) \underset{k r \gg 1}{\rightarrow} \sqrt{\frac{2}{\pi x}}(-i)^{m} e^{-i(x-\pi / 4)}
\end{aligned}
$$

we see that the $H_{m}^{(1)}\left(k_{r} r\right)$ solution corresponds to a traveling cylindrical wave propagating outward away from the origin $r=0$ while the $H_{m}^{(2)}\left(k_{r} r\right)$ is a cylindrical wave traveling inward toward the origin. 
In order to impose the boundary conditions, the total acoustic pressure field is split into three components

$$
p_{\text {total }}=p_{\text {inc }}+p_{\text {scat }}+p_{\text {int }}
$$

where $p_{\text {inc }}$ is the incident pressure, $p_{\text {scat }}$ is the scattered pressure, and $p_{\text {int }}$ is the internal pressure in the scatterer, in this case, an infinitely long circular cylinder of radius $a$. Likewise, each of these pressure fields has a unique particle velocity $\mathbf{u}_{\text {inc }}$, $\mathbf{u}_{\text {scat }}$, and $\mathbf{u}_{\text {int }}$ related through Eq. (2.6). The boundary conditions for this problem require that the pressure be continuous at the cylinder boundary $r=a$

$$
p_{\text {inc }}(a)+p_{\text {scat }}(a)=p_{\text {int }}(a)
$$

and the radial component of the particle velocity, $u_{r}$, also be continuous at $r=a$

$$
u_{r_{\text {inc }}}(a)+u_{r_{\text {scat }}}(a)=u_{r_{\text {int }}}(a)
$$

By expanding the incident field in terms of the Bessel functions and picking the appropriate general from for the internal and scattered fields, Rayleigh [19] and Morse and Ingard [20] solve for the scattered field from an infinitely long circular cylinder by matching the fields at the boundary using Eqs. (2.19) and (2.20). However, their solutions are limited to either fluid body cylinders where the assumptions in Eqs. (2.1), (2.2), and (2.3) are valid or for the perfectly rigid cylinder case where the internal particle velocity is identically zero and Eq. (2.20) becomes

$$
u_{r_{\text {inc }}}(a)+u_{r_{\text {scat }}}(a)=0 .
$$

\subsubsection{Exact modal series solution for undeformed infinitely long elastic cylinder}

The more realistic problem of scattering from an elastic cylinder is complicated by the fact that elastic bodies support shear as well as compressional waves. Faran 
[24] extended the solution method in Section 2.1 .1 by deriving the internal particle displacements from the scalar and vector potentials for small motions in an isotropic elastic solid body. Love [25] showed the equation of motion for a solid elastic medium may be written

$$
(\lambda+2 \mu) \nabla \Delta-\mu \nabla \times(2 \varpi)=\rho_{1} \frac{\partial^{2} \mathrm{~d}}{\partial t^{2}}
$$

where $\lambda$ and $\mu$ are the Lamé elastic constants, $\rho_{1}$ is the density of the elastic medium, $\mathbf{d}$ is the particle dispacement (Love and Faran use $\mathbf{u}$ ),

$$
\Delta \equiv \nabla \cdot \mathbf{d}
$$

and

$$
2 \varpi \equiv \nabla \times \mathbf{d}
$$

Taking the divergence of Eq. (2.22)

$$
\nabla^{2} \Delta=\frac{\rho_{1}}{\lambda+2 \mu} \frac{\partial^{2} \Delta}{\partial t^{2}}
$$

and the curl

$$
\nabla^{2}(2 \varpi)=\frac{\rho_{1}}{\mu} \frac{\partial^{2}(2 \varpi)}{\partial t^{2}}
$$

define a compressional wave velocity

$$
c_{l}=\sqrt{(\lambda+2 \mu) / \rho_{1}}
$$

and a shear wave velocity

$$
c_{s}=\sqrt{\mu / \rho_{1}} \text {. }
$$

In order to solve Eq. (2.22), Faran assumes the displacement potential can be expressed as a sum of a scalar and vector component

$$
\mathbf{d}=-\nabla \Psi+\nabla \times \mathbf{A}
$$

where $\nabla \cdot \mathbf{A}=0$. Thus $\Delta \equiv \nabla \cdot \mathbf{d}=-\nabla^{2} \Psi$ and $2 \varpi \equiv \nabla \times \mathbf{d}=-\nabla^{2} \mathbf{A}$ and Eqs. (2.25) and (2.26) become

$$
\nabla^{2} \Psi=\frac{1}{c_{l}^{2}} \frac{\partial^{2} \Psi}{\partial t^{2}}
$$




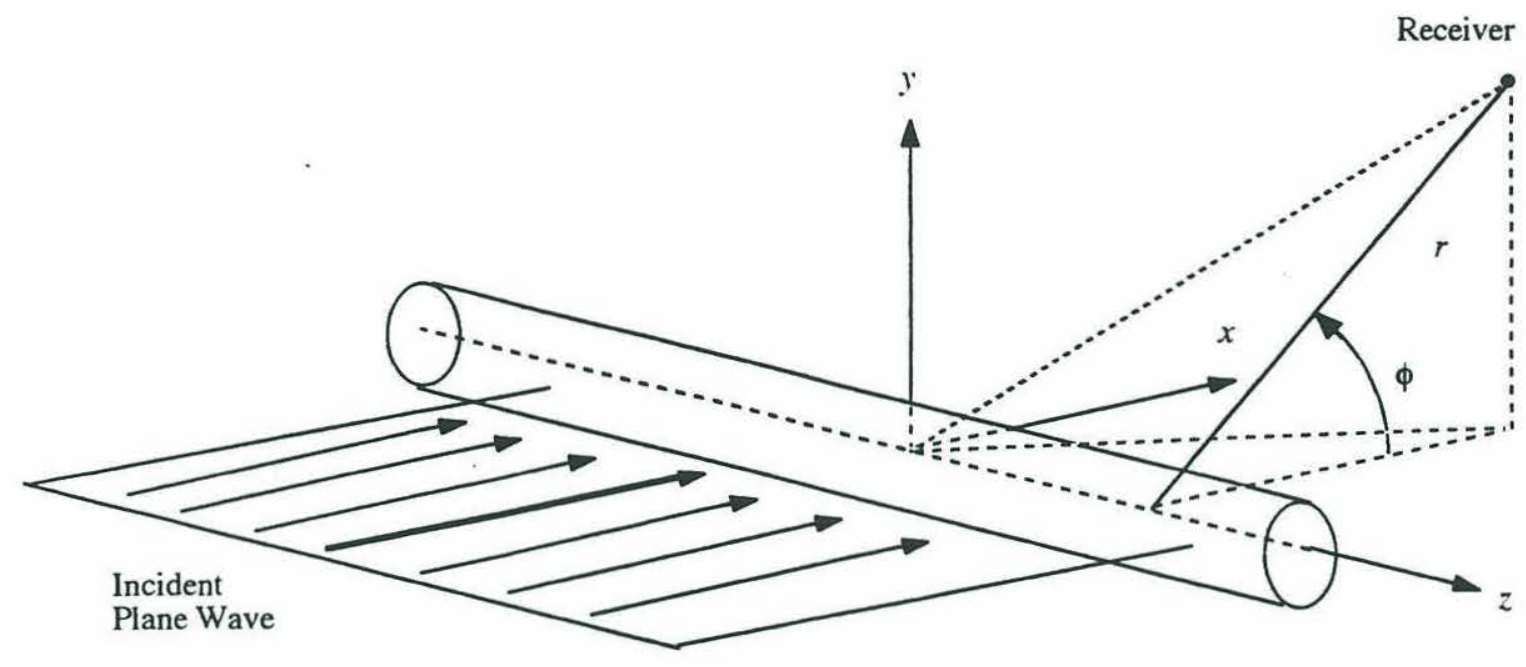

Figure 2-1: General scattering geometry for an infinitely long cylinder.

and

$$
\nabla^{2} \mathbf{A}=\frac{1}{c_{s}^{2}} \frac{\partial^{2} \mathbf{A}}{\partial t^{2}}
$$

respectively. From this, one can see that the scalar potential $\Psi$ is associated with the compressional waves in the elastic body and the vector potential $\mathbf{A}$ with the shear waves.

For an infinitely long elastic cylinder whose axis is aligned with the $z$ axis and normally incident plane waves traveling in the positive $x$ direction (Fig. 2-1), the solution should be independent of $z$ and symmetric about $\phi=0$. Since the internal displacements must be finite at $r=0$ the solution of Eq. (2.30) can be written from Eqs. (2.12) and (2.13)

$$
\Psi=\sum_{m=0}^{\infty} a_{m} J_{m}\left(k_{1} r\right) \cos m \phi
$$

where $k_{1}=\omega / c_{l}$; the time dependence $e^{-i \omega t}$ is implied and will be suppressed for the remainder of this work. We have changed Faran's $e^{i \omega t}$ convention to $e^{-i \omega t}$ resulting in a formulation that is the complex conjugate of his original equations. Since $\mathbf{d}$ should be independent of $z$, Eq. (2.29) shows that the vector potential $\mathbf{A}$ should have no $r$ or $\phi$ component. Also the vector potential must be anti-symmetric about 
$\phi=0$ in order that the radial displacement be symmetric about $\phi=0$, thus the solution of Eq. (2.31) can be written in the form

$$
\mathbf{A}=\sum_{m=0}^{\infty} b_{m} J_{m}\left(k_{2} r\right) \sin m \phi \hat{z}
$$

where $k_{2}=w / c_{s}$. Now from Eqs. (2.29), (2.32), and(2.33)

$$
\begin{aligned}
& d_{r}=\sum_{m=0}^{\infty}\left[\frac{m b_{m}}{r} J_{m}\left(k_{2} r\right)-a_{m} \frac{\partial}{\partial r} J_{m}\left(k_{1} r\right)\right] \cos m \phi, \\
& d_{\phi}=\sum_{m=0}^{\infty}\left[\frac{m a_{m}}{r} J_{m}\left(k_{1} r\right)-b_{m} \frac{\partial}{\partial r} J_{m}\left(k_{2} r\right)\right] \sin m \phi
\end{aligned}
$$

and using Eq. (2.30)

$$
\Delta=-\nabla^{2} \Psi=\frac{-1}{c_{l}^{2}} \frac{\partial^{2} \Psi}{\partial t^{2}}=k_{1}^{2} \Psi=k_{1}^{2} \sum_{m=0}^{\infty} a_{m} J_{m}\left(k_{1} r\right) \cos m \phi .
$$

The incident plane wave field of amplitude $P_{o}$ can be expanded as a series of cylindrical waves $[21]$

$$
p_{\text {inc }}=P_{o} e^{i k x}=P_{o} e^{i k r \cos \phi}=P_{o} \sum_{m=0}^{\infty} \epsilon_{m} i^{m} J_{m}(k r) \cos (m \phi)
$$

where the Neumann factor is $\epsilon_{0}=1$ and $\epsilon_{m}=2$ for $m=1,2,3, \ldots$, and $k$ is the wavenumber in the fluid. The incident field is expanded only in $J_{m}(k r)$ since it must be well behaved at the origin. The scattered field must also be symmetric about $\phi=0$ and independent of $z$, therefore from Eqs. (2.12) and (2.13) is of the form

$$
p_{\text {scat }}=\sum_{m=0}^{\infty} C_{m} H_{m}^{(1)}(k r) \cos (m \phi)
$$

where the coefficients $C_{m}$ are undetermined. The function $H_{m}^{(1)}(k r)$ is chosen in Eq. (2.38) since the scattered field is only defined for $r>a$ and should be traveling outward, away from the cylinder.

The boundary conditions (Eqs. 2.19 and 2.20) discussed for the fluid body cylinder case must be modified for scattering from an elastic body. First, for consistency 
with Faran, we consider particle displacements rather than particle velocities and Eq. (2.6) becomes

$$
\mathrm{d}=\frac{-i}{c^{2} k^{2} \rho} \nabla p .
$$

The radial components of particle displacement associated with the incident and scattered fields are

$$
d_{r_{\text {inc }}}=\frac{P_{o}}{\rho k c^{2}} \sum_{m=0}^{\infty} \epsilon_{m} i^{m} J_{m}^{\prime}(k r) \cos (m \phi)
$$

and

$$
d_{r_{\text {scat }}}=\frac{1}{\rho k c^{2}} \sum_{m=0}^{\infty} C_{m} H_{m}^{(1)^{\prime}}(k r) \cos (m \phi)
$$

respectively, where the prime indicates differentiation with respect to the function's argument. Faran [24] lists the boundary conditions appropriate for an elastic scatterer as :

1. The pressure in the fluid must be equal to the normal component of stress in the solid at the interface.

2. The normal component of displacement in the fluid must equal the normal component of displacement in the solid at the interface.

3. The tangential components of shear stress in the solid must vanish at the surface.

For our cylindrical case, these become

$$
\begin{gathered}
p_{\text {inc }}(a)+p_{\text {scat }}(a)=-\left.[r r]\right|_{r=a}, \\
d_{r_{\text {inc }}}(a)+d_{r_{\text {scat }}}(a)=d_{r}(a),
\end{gathered}
$$

and

$$
\left.[r \phi]\right|_{r=a}=\left.[r z]\right|_{r=a}=0
$$

where the stress components are given by $[24,25]$.

$$
[r r]=\lambda \Delta+2 \mu \frac{\partial d_{r}}{\partial r},
$$




$$
[r \phi]=\mu\left[\frac{1}{r} \frac{\partial d_{r}}{\partial \phi}+r \frac{\partial}{\partial r}\left(\frac{d_{\phi}}{r}\right)\right]
$$

and

$$
[r z]=\mu\left[\frac{\partial d_{r}}{\partial z}+\frac{\partial d_{z}}{\partial r}\right] .
$$

Faran solves Eqs. (2.42) - (2.44) for $C_{m}$ using Eqs. (2.34) - (2.38), (2.40), (2.41), $(2.45)-(2.47)$ and shows

$$
C_{m}=-P_{o} \epsilon_{m} i^{(m+1)} \sin \eta_{m} e^{-i \eta_{m}}
$$

where

$$
\begin{gathered}
\tan \eta_{m}=\left[\tan \delta_{m}(x)\right]\left[\tan \Phi_{m}+\tan \alpha_{m}(x)\right] /\left[\tan \Phi_{m}+\tan \beta_{m}(x)\right], \\
\tan \delta_{m}(x)=-J_{m}(x) / N_{m}(x), \\
\tan \alpha_{m}(x)=-x J_{m}^{\prime}(x) / J_{m}(x), \\
\tan \beta_{m}(x)=-x N_{m}^{\prime}(x) / N_{m}(x), \\
\tan \Phi_{m}(x)=\left(-\rho / \rho_{1}\right) \tan \zeta_{m}\left(x_{1}, \sigma\right), \\
\left.\left.\tan \zeta_{m}\left(x_{1}, \sigma\right)=\quad \frac{x_{2}^{2}}{2}\right]\right) \\
-\frac{x_{2}^{2}}{2}\left(\left[\tan \alpha_{m}\left(x_{1}\right)\right] /\left[\tan \alpha_{m}\left(x_{1}\right)+1\right]-m^{2} /\left[\tan \alpha_{m}\left(x_{2}\right)+m^{2}-\right.\right. \\
\div\left(\left[\tan \alpha_{m}\left(x_{1}\right)+m^{2}-\frac{x_{2}^{2}}{2}\right] /\left[\tan \alpha_{m}\left(x_{1}\right)+1\right]\right. \\
\left.-m^{2}\left[\tan \alpha_{m}\left(x_{2}\right)+1\right] /\left[\tan \alpha_{m}\left(x_{2}\right)+m^{2}-\frac{x_{2}^{2}}{2}\right]\right)
\end{gathered}
$$

and

$$
\begin{gathered}
x=k a, \\
x_{1}=k_{1} a, \\
x_{2}=k_{2} a .
\end{gathered}
$$


From Eq. (2.38) the exact modal solution for the scattering from an infinitely long elastic cylinder is then

$$
p_{\text {scat }}=-P_{o} \sum_{m=0}^{\infty} \epsilon_{m} i^{(m+1)} \sin \eta_{m} e^{-i \eta_{m}} H_{m}^{(1)}(k r) \cos (m \phi)
$$

and using the asymptotic expansion for the Hankel function, Eq. (2.16), the farfield limit of the scattered pressure is

$$
p_{\text {scat }} \underset{k r \gg 1}{\rightarrow}-i P_{o} \sqrt{\frac{2}{\pi k r}} e^{i(k r-\pi / 4)} \sum_{m=0}^{\infty} \epsilon_{m} \sin \eta_{m} e^{-i \eta_{m}} \cos (m \phi)
$$

\subsubsection{Simplified ray solution for infinitely long elastic cylin- der}

While the normal mode or partial wave series solution derived in Section 2.1.2 is a compact representation of the scattering field, it is computational intensive and offers little physical insight into the actual scattering processes. Recently, much attention has been given to an equivalent ray or Sommerfeld-Watson transform (SWT) formulation for convex elastic bodies $[26,27,28,29,30,31,32,33,34,35]$. This approach has the advantage of "decomposing" the scattered process into a simple superposition of physically observable wave types. The transform technique involves rewriting the partial wave series solution Eq. (2.49) as a sum of two contour integrals in the complex plane. The poles of the first integrand are found and identified with unique wave types (i.e. Rayleigh wave, Franz wave, etc.). The contour is then deformed around these poles and the integral evaluated using Cauchy's Residue Theorem $[26,28,29,30]$. The second integral is expanded as a Debye series and evaluated using the saddle point method giving the specular reflection and transmitted wave (including possible mode conversions) contributions [27]. The details of the SWT method will not be reproduced here, but a general outline of the results will aid our understanding of the observed scattered field.

Typically, the scattered field $\left(f_{S W T}\right)$ is interpreted in terms of an initial specularly reflected ray $\left(f_{s}\right)$, internally transmitted and refracted bulk rays $\left(f_{t w}\right)$, Rayleigh 
$\left(f_{R}\right)$ and Whispering Gallery $\left(f_{W G}\right)$ surface elastic waves, and Franz creeping waves $\left(f_{F}\right)$

$$
f_{S W T} \cong f_{s}+f_{t w}+f_{R}+\sum_{l=W G, F} f_{\lambda_{l}} .
$$

The specular ray is energy reflecting directly off the front interface of the cylinder. For the limit $k a \gg 1$, it can be written in a simple approximate form $[17,32]$

$$
f_{s} \approx R e^{-i 2 k a}
$$

where $R$ is the plane-wave/plane-interface Rayleigh reflection coefficient

$$
R=\frac{\rho_{1} c_{l}-\rho c}{\rho_{1} c_{l}+\rho c} .
$$

The phase shift accounts for the shortened acoustic ray path relative to a reference ray traveling to the cylinder axis and back (Fig. 2-2).

The Rayleigh and Whispering Gallery surface elastic waves represent energy coupling into the cylinder and circumnavigating the diameter while reradiating back into the fluid. Rayleigh wave speeds, $c_{R}$, depend on the locations of poles (and hence the elastic material properties) in the SWT solution but $c_{R} / c_{s}=0.91 \pm 6 \%$ provides a first order approximation [35]. These surface waves travel very fast with little attenuation and can circle the cylinder many times before decaying. Figure 2-2 illustrates the path of the first, $m=0$, Rayleigh wave. Higher order waves simply represent multiple passes around the cylinder. The angle at which energy couples between the fluid and elastic is governed by well known trace velocity principles. Coupling occurs when the Rayleigh wave speed in the elastic $c_{R}$ matches the component of the incident wave phase velocity along the cylinder surface $c / \sin \theta$ where $\theta$ is the local angle of incidence. Therefore, the Rayleigh "launch" angle is

$$
\theta_{R}=\arcsin \left(c / c_{R}\right)
$$

The phase shift for the $m=0$ surface wave is

$$
\eta_{R}=k a\left[\left(c / c_{R}\right)\left(2 \pi-2 \theta_{R}\right)-2 \cos \theta_{R}\right]
$$




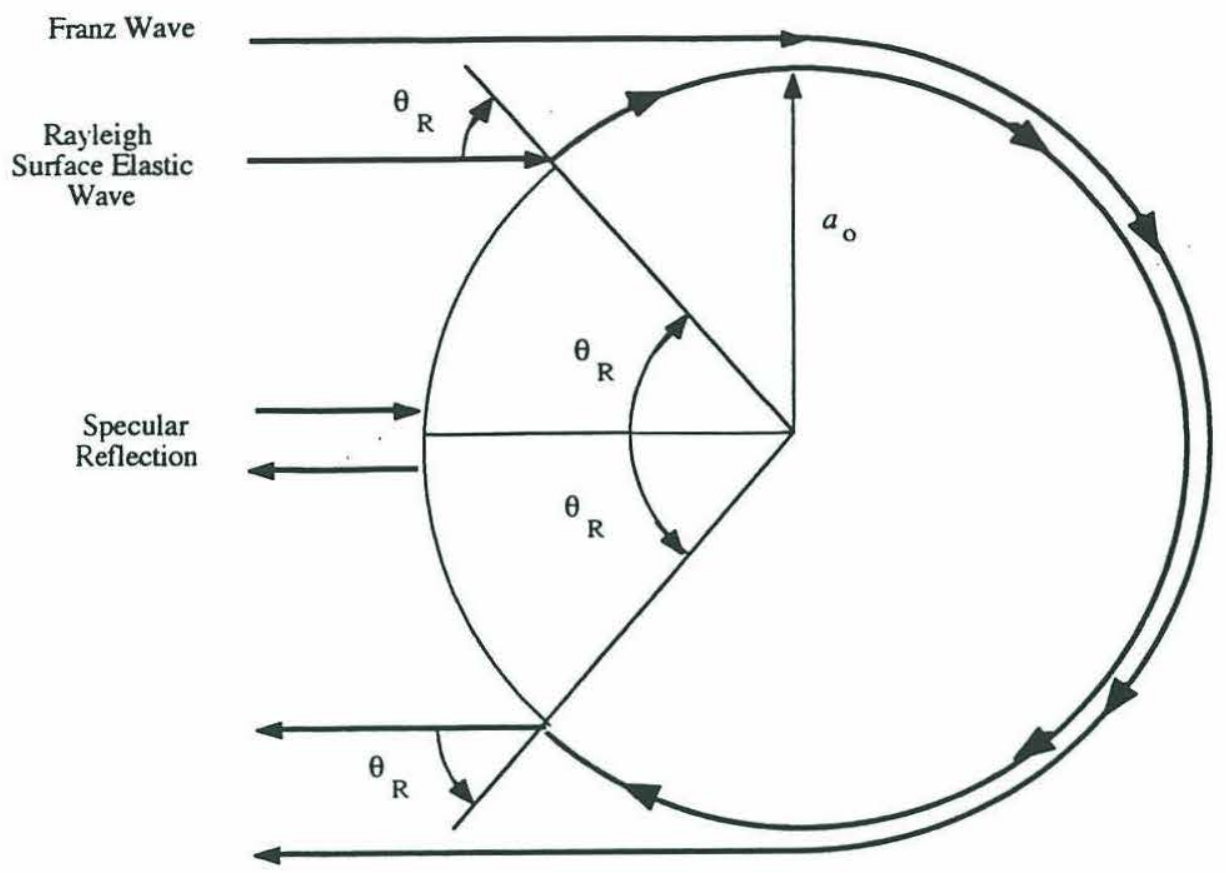

Figure 2-2: "Ray" diagram implied by Sommerfeld-Watson transformation showing the specular, $m=0$ Rayleigh and Franz wave contributions to backscattering from elastic cylinders.

where the first term is the phase for the acoustic path in the cylinder and the second is the phase for the shortened path in the fluid relative to a reference ray traveling to and from the cylinder's axis. The approximate expression for the Rayleigh contribution is then $[17,32,33,34]$

$$
f_{R} \approx-G_{R} e^{-2\left(\pi-\theta_{R}\right) \beta_{R}} e^{i \eta_{R}} \sum_{m=0}^{\infty} e^{-2 \pi m \beta_{R}} e^{i 2 \pi m k a c / c_{R}} .
$$

The radiation damping term $\beta_{R}$ accounts for the continual reradiation of energy into the fluid as the surface wave encircles the cylinder. Note that this approximate form assumes a constant Rayleigh wave speed and neglects dispersive effects. For cylinders, the complex coupling coefficient $G_{R}$ can be written in the approximate form $[17,34]$

$$
G_{R} \approx 8 \pi \beta_{R} e^{i \Phi_{R}} / \sqrt{\pi k a} .
$$

The other circumferential waves can be written in a similar form. 
The Franz creeping wave also circumnavigates the cylinder but the energy is concentrated almost entirely in the fluid. The phase velocity is typically less than the phase velocity of the fluid, thus the term creeping wave when compared to the fast surface elastic wave (some authors use the term creeping wave for all the circumferential waves, however we will reserve it for the Franz contribution).

The structure of the frequency response in the backscattered field can now be interpreted in terms of the interference patterns generated by the various waves. Sharp nulls appear in the scattered form function when one or more of these waves are out of phase and destructively interfere. For dense elastic solids and moderately high $k a$, the specular and Rayleigh waves dominate the scattered field $[17,31]$ and the approximate ray solution just considers these two wave types:

$$
f_{S W T a p p r o x} \approx f_{s}+f_{R} .
$$

\subsubsection{Deformed cylinder formulation}

In a series of papers, Stanton $[14,15,16]$ developed an approximate model for scattering from elongated objects deformed along their major axis by making an analogy between the far-field scattered pressure and the radiated pressure from a continuous line source. Skudrzyk [21] shows that the sound pressure measured a distance $r$ from a point monopole source can expressed in terms of a volume flow $\mathrm{Q}$

$$
p_{\text {monopole }}=\frac{-i k \rho c}{4 \pi r} Q e^{i k r}
$$

(we have changed Skudrzyk's $e^{-i k r}$ convention to $e^{i k r}$ resulting in a solution that is the complex conjugate of his). A line source can likewise be considered a continuous distribution of point monopoles and Skudrzyk gives the differential pressure from such a line source as

$$
d p_{\mathrm{ls}}=\frac{-i k \rho c}{4 \pi r_{s}} q e^{i k r \cdot} d z
$$

where $q$ is the volume flow per unit length of the line source and $r_{s}$ is the distance from the receiver (or field point) to an arbitrary differential source element. 
Integrating over the line source gives the total pressure

$$
p_{\mathrm{ls}}=\frac{-i k \rho c}{4 \pi} \int_{-\infty}^{\infty} \frac{e^{i k r_{0}}}{r_{s}} d z
$$

Skudrzyk transforms and evaluates the integral in Eq. (2.61)

$$
\int_{-\infty}^{\infty} \frac{e^{i k r_{0}}}{r_{s}} d z=i \pi H_{0}^{(1)}(k r)
$$

where $r$ is the perpendicular distance from the receiver to the line source $\left(r_{s} \geq r\right)$. Therefore

$$
p_{\mathrm{ls}}=\frac{1}{4} k \rho c q H_{0}^{(1)}(k r)
$$

and using Eq. (2.16), the pressure in the far-field is

$$
p_{\mathrm{ls}} \underset{k r \gg 1}{\rightarrow} \frac{1}{4} k \rho c q \sqrt{\frac{2}{\pi k r}} e^{i(k r-\pi / 4)} .
$$

Stanton notes that the radiated pressure due to the line source in Eq. (2.64) is similar in form to the scattered pressure from infinitely long cylinder in Eq. (2.50). Since scattering is in essence the reradiation of the incident sound field by the scattering body, an infinitely long cylinder is effectively a line source producing some volume flow. This analogy is limited in that a line source produces an omnidirectional field with respect to $\phi$ while Eq. (2.50) indicates that the scattered field from a cylinder is highly directional. Equating Eqs. (2.50) and (2.64), one derives $q_{a}$, an apparent volume flow per unit length for the infinitely long elastic cylinder

$$
q_{a}=\frac{-4 i P_{o}}{k \rho c} \sum_{m=0}^{\infty} \epsilon_{m} \sin \eta_{m} e^{-i \eta_{m}} \cos (m \phi)
$$

as observed in the $\phi$ direction. Modifying Eq. (2.60), Stanton derives an approximate generalized formula for the scattered pressure per unit length for an arbitrary geometry

$$
d p_{\text {scat }}=\frac{-i k \rho c}{4 \pi r_{s}} q_{a} e^{i k\left(r_{o}+\epsilon_{o}\right)}\left|d \mathbf{r}_{\text {pos }}\right|
$$

where $r_{p o s}$ is the position vector of a differential element on the cylinder axis, $r_{s}$ is the distance from the element at $r_{\text {pos }}$ to the receiver (or field) point, and $\epsilon_{s}$ is 


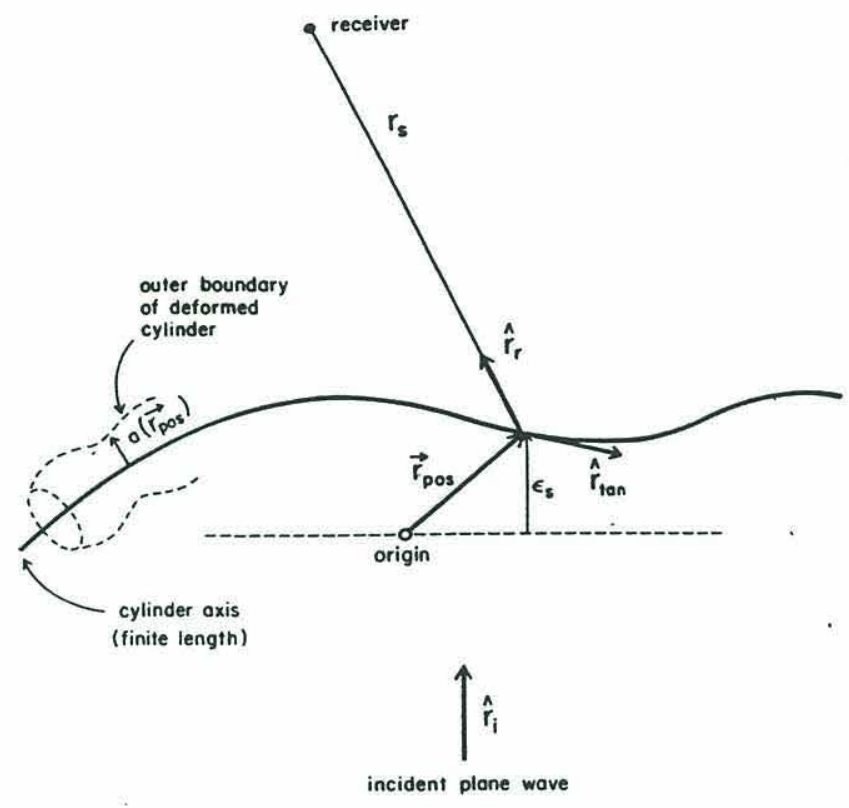

Figure 2-3: Scattering geometry for a plane wave incident upon a deformed finite cylinder with a point receiver. From Stanton [16].

the distance between the element on the axis and the plane that is normal to the incident wave and contains the origin; see Fig. 2-3 (phase correction factor included since the cylinder is no longer constrained to the $z$ axis). Integrating over the cylinder gives the total scattered pressure

$$
p_{\mathrm{scat}_{\mathrm{dc}}}=\frac{-i k \rho c}{4 \pi} \int_{\mathbf{r}_{\mathrm{pos}}} q_{a} \frac{e^{i k\left(r_{o}+\epsilon_{o}\right)}}{r_{s}}\left|d \mathbf{r}_{\mathrm{pos}}\right| .
$$

Inserting $q_{a}$ from Eq. (2.65) gives an approximate form for scattering from an elastic cylinder

$$
p_{\text {scat }_{\mathrm{dc}}} \cong \frac{-1}{\pi} \int_{\mathbf{r}_{\mathrm{pos}}} P_{o} \frac{e^{i k\left(r_{\bullet}+\epsilon_{o}\right)}}{r_{s}} \sum_{m=0}^{\infty} \epsilon_{m} \sin \eta_{m} e^{-i \eta_{m}} \cos (m \phi)\left|d \mathbf{r}_{\mathrm{pos}}\right|
$$

where the wavenumbers in the modal summation factor are multiplied by the sine of the angle between the direction of the incident wave $\hat{r}_{i}$ and the tangent to the axis $\hat{r}_{\tan }$ (i.e. replace all $k$ in $\eta_{m}$ by $\left.k\left|\hat{r}_{i} \times \hat{r}_{\tan }\right|\right)$. 


\subsubsection{Modification for point source and receiver with arbi- trary directivity patterns}

Although Eq. (2.68) was derived assuming an incident plane wave, DiPerna and Stanton [36] generalize the solution to include point sources by treating the incident field at each differential element on the cylinder as locally plane. Equation (2.65) can be modified to account for a spherically spreading field of a point source by making $P_{o}$ a function of position

$$
P_{o}\left(\mathrm{r}_{\mathrm{pos}}\right)=\frac{P_{o} r_{o}}{r_{i}} e^{i k r_{i}}
$$

where $\mathbf{r}_{i}$ is a vector from the source to the point on the cylinder axis at $\mathbf{r}_{\mathrm{pos}}$, $r_{i}=\left|\mathbf{r}_{i}\right|$, and $P_{o}$ is the incident wave amplitude measured at a reference distance $r_{o}$. To accommodate the experiment described later in this thesis, we include gain functions in Eq. (2.69) associated with the source radiation pattern and receiver directivity response pattern $[2]$

$$
P_{o}\left(\mathrm{r}_{\mathrm{pos}}\right)=\frac{P_{o} r_{o}}{r_{i}} D\left(\theta_{i}, \hat{r}_{\mathrm{src}}\right) D\left(\theta_{s}, \hat{r}_{\mathrm{rcv}}\right) e^{i k r_{i}}
$$

where $D(\theta, \hat{r})$ is the amplitude factor at an angle $\theta$ from the maximum response axis $\hat{r}, \hat{r}_{\text {src }}$ and $\hat{r}_{\text {rev }}$ are the maximum response axes for the source and receiver respectively, $\theta_{i}$ is the angle between $r_{i}$ and $\hat{r}_{\text {src }}$, and $\theta_{s}$ is the angle between $r_{s}$ and $\hat{r}_{\text {rev }}$ (Fig. 2-4). The gain functions are normalized such that $D(\theta=0, \hat{r})=1$. Inserting Eq. (2.70) into Eq. (2.65), substituting this into Eq. (2.67), and omitting $\epsilon_{m}$ which is now implicitly included in $r_{i}$, the general expression for scattering of an incident spherically spreading wave with arbitrary beam pattern by a deformed elastic cylinder is

$$
\begin{gathered}
p_{\text {scat }_{\mathrm{dc}}} \cong \frac{-P_{o} r_{o}}{\pi} \int_{\mathbf{r}_{\mathrm{pos}}} \frac{e^{i k\left(r_{i}+r_{o}\right)}}{r_{i} r_{s}} D\left(\theta_{i}, \hat{r}_{\mathrm{src}}\right) D\left(\theta_{s}, \hat{r}_{\mathrm{rcv}}\right) \times \\
\sum_{m=0}^{\infty} \epsilon_{m} \sin \eta_{m} e^{-i \eta_{m}} \cos (m \phi)\left|d \mathbf{r}_{\mathrm{pos}}\right|
\end{gathered}
$$




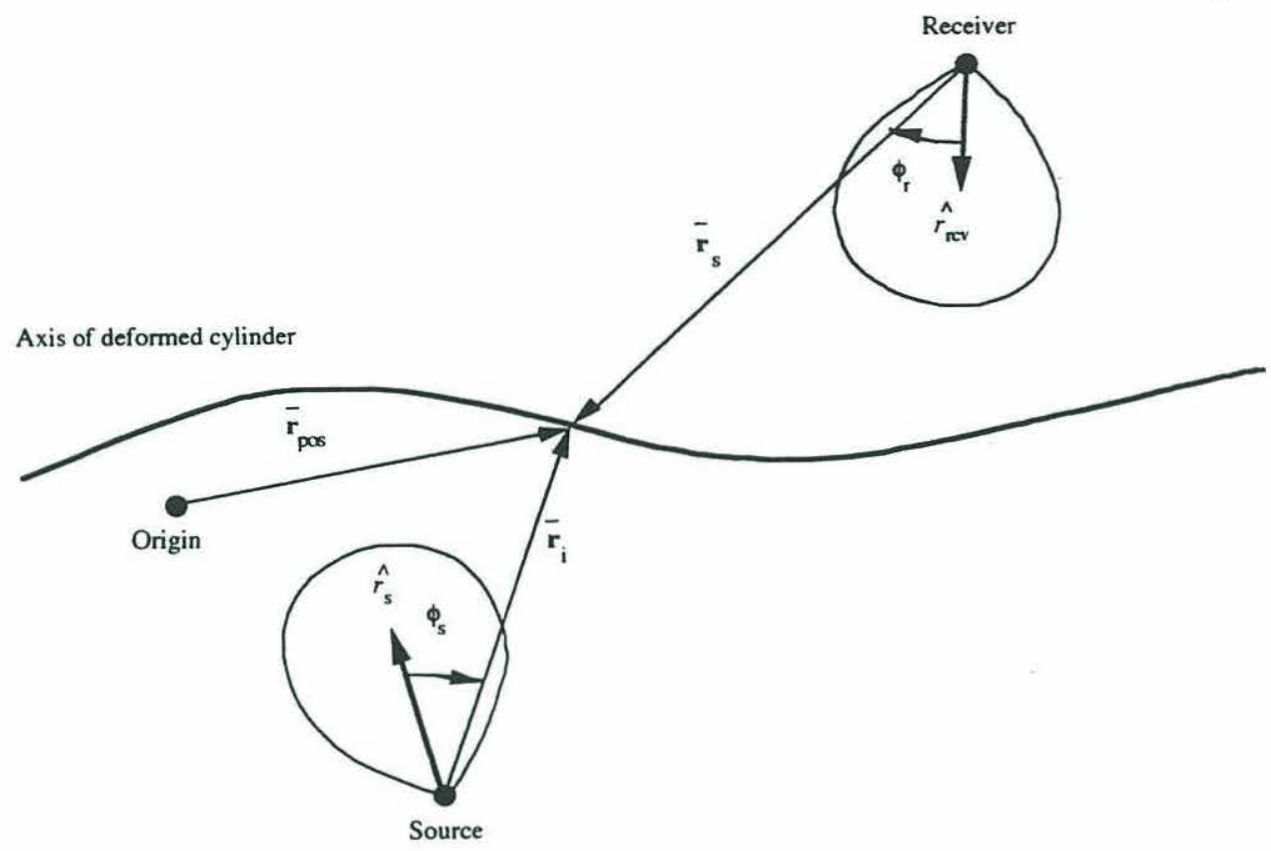

Figure 2-4: Scattering geometry for deformed cylinder and general bistatic geometry (directional transmitter and receiver).

\subsubsection{Extension to randomly rough cylinders}

Using the deformed cylinder formulation, Stanton has derived approximate solutions for scattering from a wide variety of elongated objects, each of which is modeled using Eq. (2.67) with the appropriate choice of $q_{a}$ for the material properties and $d \mathbf{r}_{\text {pos }}$ for the geometry of the scattering body. Recently, he has used this deformed cylinder formulation to analyze the effects of surface roughness on the scattered field $[17,18]$. The roughness is modeled as an axially dependent perturbation of the radius, $\zeta\left(\mathbf{r}_{\mathrm{pos}}\right)$, about some mean value $a\left(\mathbf{r}_{\mathrm{pos}}\right)$. The formulation assumes that the perturbation is slowly varying and allows for the quantitative analysis of random surface roughness on otherwise regular objects. The surface profile is given as

$$
a_{R C}\left(\mathrm{r}_{\mathrm{pos}}\right)=a\left(\mathrm{r}_{\mathrm{pos}}\right)+\zeta\left(\mathrm{r}_{\mathrm{pos}}\right) .
$$

The perturbation $\varsigma\left(\mathbf{r}_{\mathrm{pos}}\right)$ is a zero mean stochastic variable such that

$$
\left\langle\varsigma\left(\mathrm{r}_{\mathrm{pos}}\right)\right\rangle=0 \text { and }\left\langle a_{R C}\left(\mathrm{r}_{\mathrm{pos}}\right)\right\rangle=a\left(\mathrm{r}_{\mathrm{pos}}\right) .
$$




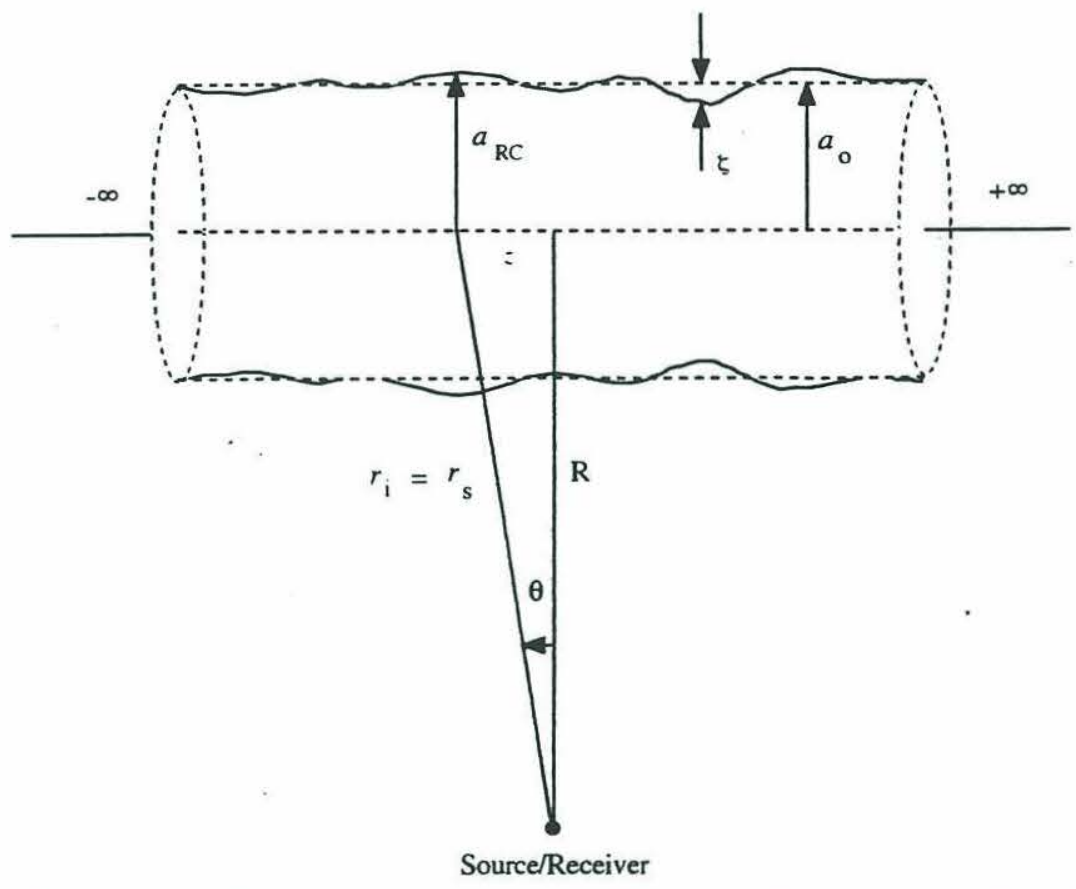

Figure 2-5: Geometry for (monostatic) backscatter from a rough infinitely long cylinder aligned along $z$ axis. The mean radius $a_{o}$ and perturbation, $\varsigma(z)$, component of the surface profile $a_{R C}(z)$ are shown.

Adding surface roughness to a straight circular cylinder aligned along the $z$ axis with constant radius $a_{o}$ yields (Fig. 2-5)

$$
\begin{aligned}
a_{R C}(z) & =a_{o}+\zeta(z) \\
\left\langle a_{R C}(z)\right\rangle & =a_{o}
\end{aligned}
$$

\subsection{Explicit solution for rough infinitely long cylin- ders}

Given the deformed formulation in Section 2.1, we can derive an approximate analytical result for backscattering from a randomly rough elastic cylinder. Considering the monostatic case where the source/receiver is at a distance $R$ from the cylinder axis which is aligned with the $z$ axis and where the directivity response patterns are 
confined to relatively small angles, we approximate the gain functions as Gaussian with characteristic beamwidth $\Theta_{0}[37]$

$$
D\left(\theta_{i}, \hat{r}_{\mathrm{src}}\right)=D\left(\theta_{s}, \hat{r}_{\mathrm{rcv}}\right)=e^{-\left((2 \theta)^{2} / 2 \theta_{o}^{2}\right)} .
$$

The maximum response axis $\hat{r}_{\mathrm{src} / \mathrm{rcv}}$ is aligned normal to the cylinder axis and along the $x$ axis (Fig. 2-5). Thus setting $\theta=\arctan (z / R), \phi=\pi, r_{i}=r_{s}=\sqrt{R^{2}+z^{2}}$, and $\left|d \mathbf{r}_{\text {pos }}\right|=d z$, Eq. (2:71) simplifies to

$$
\begin{gathered}
p_{\text {scat }} \text { dc } \cong \frac{-P_{o} r_{o}}{\pi} \int_{-\infty}^{\infty} \frac{e^{i 2 k\left(R^{2}+z^{2}\right)^{1 / 2}}}{R^{2}+z^{2}} e^{-\left(2 \theta / \Theta_{o}\right)^{2}} \times \\
\sum_{m=0}^{\infty} \epsilon_{m}(-1)^{m} \sin \eta_{m} e^{-i \eta_{m}} d z
\end{gathered}
$$

where

$$
\eta_{m}(k a) \rightarrow \eta_{m}\left(k \cos \theta\left[a_{o}+\varsigma(z)\right]\right)=\eta_{m}\left(k\left[a_{o}+\varsigma(z)\right] / \sqrt{1+(z / R)^{2}}\right)
$$

According to Eq. (2.76), the beamwidth of the source/receiver with respect to the first Fresnel zone diameter determines whether the scattered field spreads cylindrically or spherically.

The Fresnel (or Huygens) zone is the insonified area from which the scattered waves add constructively at the receiver $[21,38,39]$. For a planar surface insonified by a point source/receiver, the first Fresnel zone is a circle with diameter

$$
\text { F.Z. diameter }=\sqrt{2 r \lambda}
$$

where $r$ is the perpendicular distance form the insonified plane to the source/receiver point. In scattering theory, the length of a cylinder with respect to the first Fresnel zone diameter determines the characteristic spreading law of the scattered field $[14,36,39,40]$. If the cylinder is much longer than the first Fresnel zone, the cylinder may be considered infinite and the scattered field spreads cylindrically from the target. If the cylinder length is small compared to the first Fresnel zone, then it is considered finite and the scattered field spreads spherically. In the case of a spherically spreading incident field with a narrow beam, the beamwidth $\Theta_{0}$ determines 
the effective length of the cylinder. If $\Theta_{0}$ is very narrow such that the segment of the cylinder insonified by the source is less than the first Fresnel zone diameter, the backscattered field will spread spherically (even though the cylinder itself may be much long than the first Fresnel zone). Likewise if the beamwidth is wide enough to span several Fresnel zones (but not the ends of the cylinder) the cylinder is effectively infinite and the backscattered field will spread cylindrically (even though the target may be of finite length).

For this work, we will consider the last case where the beamwidth is wide enough to span several Fresnel zones and the scattered field spreads cylindrically. It is customary to introduce the far field form function $f^{\infty}$ to give a nondimensional representation of the scattered pressure for $k r \gg 1$ :

$$
p_{\text {scat }}=P_{o} \sqrt{\frac{a}{2 r}} e^{i k r} f^{\infty} .
$$

While some authors use " $\infty$ " to identify the $k r \gg 1$ case, all results presented here are for $k r \gg 1$ so the distinction is not made; the " $\infty$ " here refers to the infinite length of the cylinder. Replacing the incident plane wave field $P_{o}$ with a spherically spreading field given in Eq. (2.69), Eq. (2.79) becomes

$$
p_{\text {scat }}=\frac{P_{o} r_{o}}{r} \sqrt{\frac{a_{o}}{2 r}} e^{i 2 k R} f^{\infty} .
$$

Using Eq. (2.76), the far field form function for the infinitely long elastic cylinder is

$$
\begin{gathered}
f_{\text {rough }}^{\infty} \cong \frac{-R}{\pi} \sqrt{\frac{2 R}{a_{o}}} e^{-i 2 k R} \int_{-\infty}^{\infty} \frac{e^{i 2 k\left(R^{2}+z^{2}\right)^{1 / 2}}}{R^{2}+z^{2}} e^{-\left(2 \theta / \Theta_{o}\right)^{2}} \times \\
\sum_{m=0}^{\infty} \epsilon_{m}(-1)^{m} \sin \eta_{m} e^{-i \eta_{m}} d z
\end{gathered}
$$

where roughness effects $a_{R C}(z)$ are implicit in $\eta_{m}$. This equation represents one realization of the scattered field due to a single stochastically rough cylinder. From Eq. (2.81), we can directly write expressions for the mean and mean square scattered fields for backscatter from an ensemble of statistically independent infinitely long 
rough elastic cylinders

$$
\begin{aligned}
\left\langle\left|f_{\text {rough }}^{\infty}\right|\right\rangle \cong & \frac{R}{\pi} \sqrt{\frac{2 R}{a_{o}}}\langle| \int_{-\infty}^{\infty} \frac{e^{i 2 k\left(R^{2}+z^{2}\right)^{1 / 2}}}{R^{2}+z^{2}} e^{-\left(2 \theta / \Theta_{o}\right)^{2}} \times \\
& \sum_{m=0}^{\infty} \epsilon_{m}(-1)^{m} \sin \eta_{m} e^{-i \eta_{m}} d z|\rangle
\end{aligned}
$$

and

$$
\begin{aligned}
\left\langle\left|f_{\text {rough }}^{\infty}\right|^{2}\right\rangle \cong & \left(\frac{R}{\pi}\right)^{2} \frac{2 R}{a_{o}}\langle| \int_{-\infty}^{\infty} \frac{e^{i 2 k\left(R^{2}+z^{2}\right)^{1 / 2}}}{R^{2}+z^{2}} e^{-\left(2 \theta / \Theta_{o}\right)^{2}} \times \\
& \left.\left.\sum_{m=0}^{\infty} \epsilon_{m}(-1)^{m} \sin \eta_{m} e^{-i \eta_{m}} d z\right|^{2}\right\rangle
\end{aligned}
$$

Here $\langle\cdots\rangle$ represents the average across an ensemble of statistically independent surfaces. Evaluation of these last two equations is typically performed numerically although simplified versions of them involving ray solutions have been solved analytically to illustrate some of the basic physics of roughness-induced scattering phenomena $[17,18]$.

\subsection{Fluctuations in the scattered field}

The scattered field from a rough cylinder (Eq. 2.81) will fluctuate from realization to realization due to the random surface profile. If we consider a set of measurements involving independent realizations, the scattered field $f$ for each measurement can be expressed as the sum of a mean, $\langle f\rangle$, and fluctuating component, $\delta f$

$$
f=\langle f\rangle+\delta f
$$

where $\langle\delta f\rangle=0$

and $\langle f\rangle$ is the ensemble average across all the measurements. Figure 2-6 illustrates this relation in terms of a phasor diagram.

Many investigators have shown that the Rice probability density function (PDF) may be used to describe echo fluctuations due to surface roughness $[4,18,41]$. This 


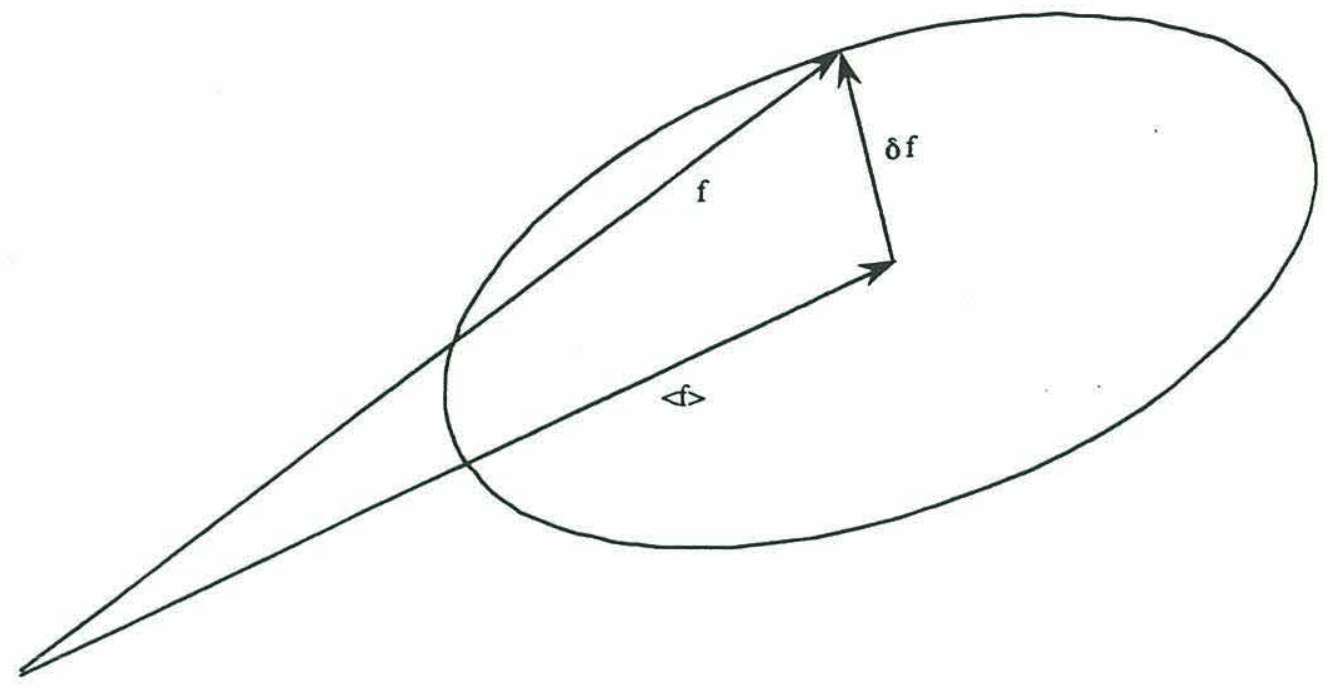

Figure 2-6: Phasor diagram showing mean and fluctuation components of echo.

distribution was originally derived to describe the statistics of the envelope of a sine wave corrupted by additive noise [42]. Rice showed that the PDF for the random amplitude $A$ of a sine wave of rms value $S$ summed with zero-mean Gaussian white noise with a mean square value of $\left\langle N^{2}\right\rangle$ is

$$
w_{R}(A)=\frac{A}{\left\langle N^{2}\right\rangle} \exp \left(-\frac{A^{2}+2 S^{2}}{2\left\langle N^{2}\right\rangle}\right) I_{0}\left(\frac{\sqrt{2} A S}{\left\langle N^{2}\right\rangle}\right)
$$

where $I_{0}$ is the modified Bessel function. Defining a shape parameter $\gamma$ as the ratio of the signal energy to the noise energy

$$
\gamma \equiv S^{2} /\left\langle N^{2}\right\rangle
$$

it is possible to rewrite Eq. (2.85) as a function of the shape parameter [4]

$$
w_{R}(A)=\frac{2 A(1+\gamma)}{\left\langle A^{2}\right\rangle} \exp \left(-\frac{(1+\gamma) A^{2}+\gamma\left\langle A^{2}\right\rangle}{\left\langle A^{2}\right\rangle}\right) I_{0}\left(q^{\prime}\right)
$$

where

$$
q^{\prime}=\frac{2 A[\gamma(1+\gamma)]^{1 / 2}}{\left\langle A^{2}\right\rangle^{1 / 2}} .
$$

Considering rough surface scattering, the mean field $\langle f\rangle$ is analogous to the sine wave or signal in the Rice PDF and the fluctuations $\delta f$ analogous to the noise [18]. 
The Rice PDF can then be used to describe the distribution of fluctuations in the scattered field

$$
w_{R}(f)=\frac{2|f|(1+\gamma)}{\left\langle|f|^{2}\right\rangle} \exp \left(-\frac{(1+\gamma)|f|^{2}+\gamma\left\langle|f|^{2}\right\rangle}{\left\langle|f|^{2}\right\rangle}\right) I_{0}\left(q^{\prime}\right)
$$

where

$$
q^{\prime}=\frac{2|f|[\gamma(1+\gamma)]^{1 / 2}}{\left\langle|f|^{2}\right\rangle^{1 / 2}} .
$$

The PDF, $w_{R}(f)\left\langle|f|^{2}\right\rangle^{1 / 2}$, is a function of $|f| /\left\langle|f|^{2}\right\rangle^{1 / 2}$ and $\gamma$ as illustrated in Fig. 2-7. The Rice distribution becomes the Raleigh PDF for $\gamma=0$ and asymptotically approaches the Gaussian distribution for $\gamma \gg 1$.

When the cylinder is very rough, the scattered field is dominated by fluctuations due to the random surface profile. This is analogous to a pure noise field and characterized by a Rayleigh-like PDF $(\gamma \ll 1)$. When the surface becomes acoustically smooth, the field fluctuates very little $(\gamma \gg 1)$ and the distribution of $\left|f^{\infty}\right|$ will become a narrow Gaussian tending toward a delta function. There is a smooth transition between these two cases for intermediate values of $\gamma$ and surface roughness. 


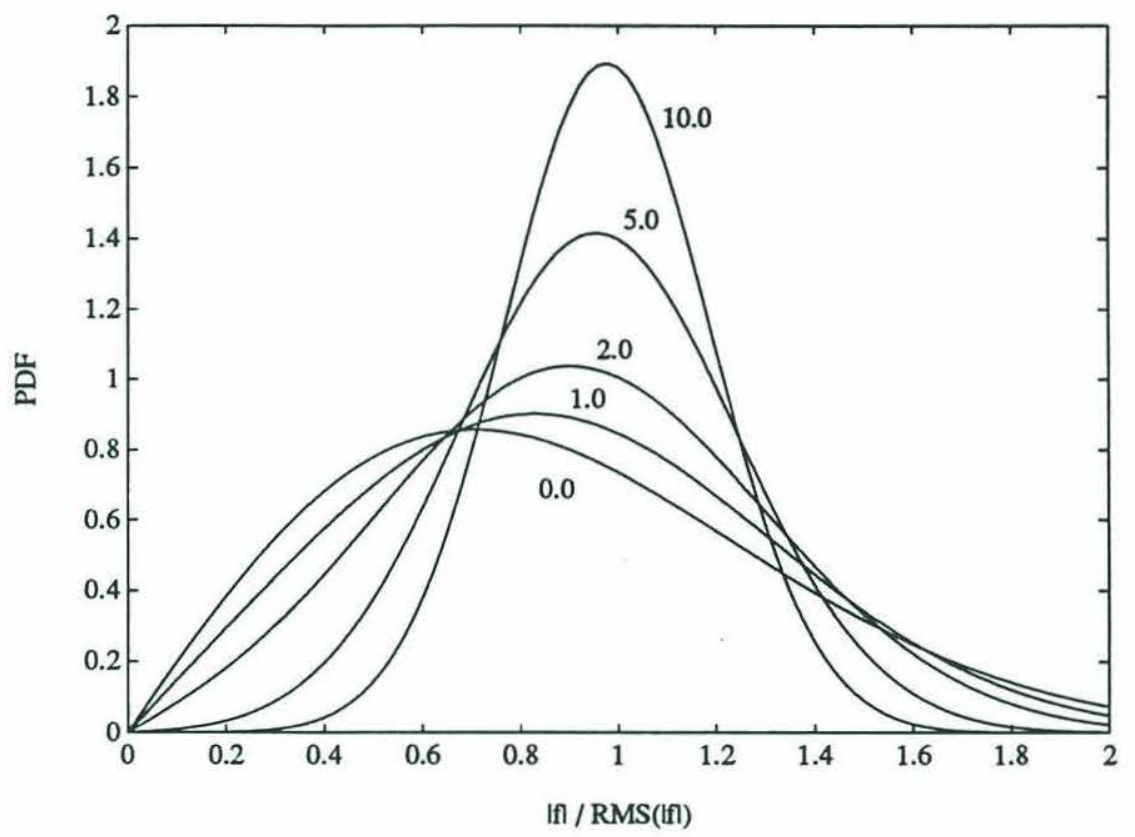

Figure 2-7: Rice PDF of scattering amplitude for various values of $\gamma$. For $\gamma=0$ the curve is the Rayleigh PDF and for $\gamma \gg 1$ the Rice PDF approaches a Gaussian. $\mathrm{PDF} \equiv w_{R}(f)\left\langle|f|^{2}\right\rangle^{1 / 2}$. 


\section{Chapter 3}

\section{Numerical Analysis}

The formulations derived in Chapter 2 for rough cylinder scattering (Eqs. 2.82 and 2.83) do not have closed form solutions but do provide a framework for numerical solution using standard techniques of numerical integration. However, before we can consider solution methods, we must first define the random surface profile, which in turn raises the questions of what do we mean by "random" and how can we deterministically model such a process.

\subsection{Modeling of surface roughness}

The formulation in Section 2.2 assumes a zero mean random perturbation which slowly varies along the axis over the local scale. A Gaussian white noise process satisfies the zero mean requirement but each point is completely decorrelated from the next. Thus the surface would contain an assortment of jagged edges and discontinuities which our model does not allow. However, a bandlimited Gaussian white noise process provides a smooth variation from one point to the next due to the cutoff of the higher frequencies (an impulsive discontinuity requires an infinite number of frequency components). This is similar to the theory of bandlimited electrical circuit noise investigated by Rice [42]. Rice showed that some forms of 
random circuit noise are bandlimited Gaussian white noise and could be modeled as a sum of random sinusoids

$$
I(t)=\sum_{n=1}^{N} c_{n} \sin \left(\omega_{n} t+\varphi_{n}\right)
$$

where $c_{n}$ is a random amplitude, $\omega_{n}$ is a random angular frequency, and $\varphi_{n}$ is a random phase. If $N$ is sufficiently large, then, from the central limit theorem, the distribution of $I(t)$ will be Gaussian. Stanton $[17,18]$ uses this formulation to model the random surface perturbations for a rough cylinder. Changing from a bandlimited random time series to a bandlimited random spatial series, the random surface perturbation is modeled by

$$
\varsigma(z)=\sigma_{s} \sqrt{6 / N} \sum_{n=1}^{N} \mu_{n} \sin \left(K_{n} z+\delta_{n}\right)
$$

where $\sigma_{s}$ is the desired rms roughness for the profile. The random amplitude coefficients $\mu_{n}$ are uniformly distributed between 0 and 1 , the spatial wavenumber components, $\mathcal{K}_{n}$, are uniformly distributed between the bandlimits $\mathcal{K}_{\text {low }}$ and $\mathcal{K}_{\text {high }}$, and the phase factors, $\delta_{n}$, are uniformly distributed between 0 and $2 \pi$. A random number generator is used to seed these variables and the factor $\sqrt{6 / N}$ (in this case $N=100$ ) is included as a normalization constant for the variance since the desired variance is $\sigma_{s}^{2}$. Figure 3-1 shows a histogram of the perturbation output from this algorithm illustrating the underlying Gaussian nature of the process. It appears as if there is a slight skew in the distribution near the peak at $\zeta=0$ but checks verified this algorithm produces a zero mean distribution (within numerical roundoff errors). This slight shift in the peak appeared in many of the simulations (Fig. 4-3).

In order to establish bandlimits for the surface profile, we must consider the criteria necessary to consider a given surface acoustically rough. Very low frequency components in the surface spectra correspond to long undulations in the profile. If these undulations are much longer than the acoustic wavelength and/or the insonified area of the target, the surface will appear acoustically flat with some nonzero slope. The coherent reflection is determined by the first Fresnel zone [39] so if the 


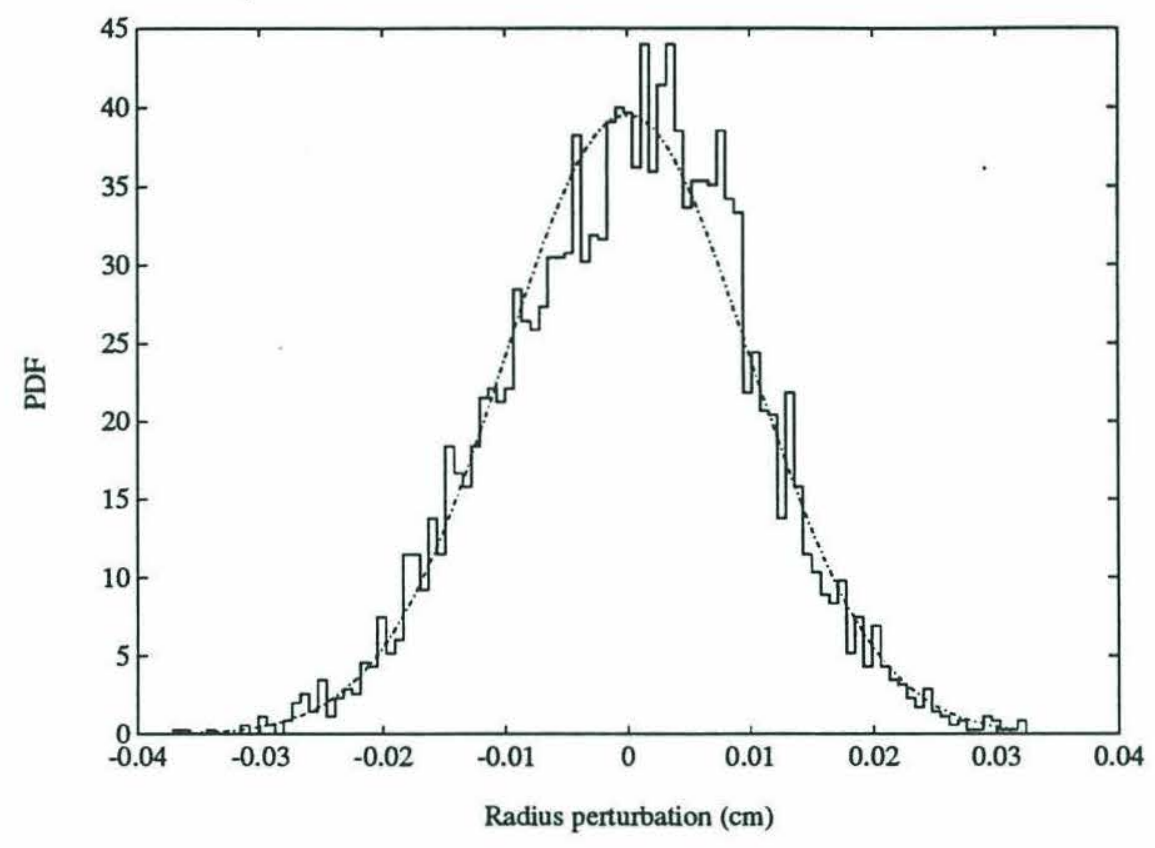

Figure 3-1: Histogram for rough surface simulation showing Gaussian nature of the profile statistics. 100 random sinusoids were summed to provide the profile. The input rms roughness $\sigma_{s}$ was $100 \mu \mathrm{m}$. The area under the histogram has been normalized to 1 to give a perturbation PDF. The superimposed Gaussian PDF was calculated with the same standard deviation $\sigma_{s}$. 
surface varies over the extent of the first Fresnel zone diameter, the surface will appear acoustically rough at that particular frequency. We arbitrarily set the surface wavenumber bandlimits, $\mathcal{K}_{\text {low }}$ and $\mathcal{K}_{\text {high }}$, such that at least ten wavelengths $(\Lambda)$ of the surface profile fit within the first Fresnel zone at all frequencies of interest:

$$
\Lambda=\frac{\sqrt{2 r \lambda}}{10}
$$

giving

$$
K_{\text {limit }}=10 \pi \sqrt{\frac{2}{r \lambda}}
$$

where $\lambda$ is the acoustic wavelength and $r$ is the source/receiver to target separation.

For a given simulation, three 100 element arrays of random numbers were generated to uniformly distribute the amplitude, wavenumber and phase variables in Eq. (3.2) over their respective bands. Then the 100 sinusoids were summed for each value of $z$ in the profile. The central section of each profile $(-25 \mathrm{~cm}<\mathrm{z}<25$ $\mathrm{cm}$ ) was calculated at intervals of $0.01 \mathrm{~cm}$ (highly oversampling the surface), and these values formed a look up table from which the perturbation could be linearly interpolated for any value of $z$.

\subsection{Evaluation of integral}

In order to chose an efficient numerical integration algorithm, we must investigate the nature of the integrand in Eq. (2.81). The infinite modal summation term in the integrand provides all the information about the resonant and scattering modes of the target. This summation quickly converges when $m \approx k a$ so we summed the first $k a+10$ terms to approximate a converged solution. Figure 3-2 illustrates a typical converged solution. The solution shows a general smooth oscillatory nature with sharp nulls where the function is not analytic (higher order derivatives do not exist).

Using this converged summation, we can analyze the integrand of the scattered field form function (Eq. 2.81) as a function of $z$. Since we have a spherically spread- 


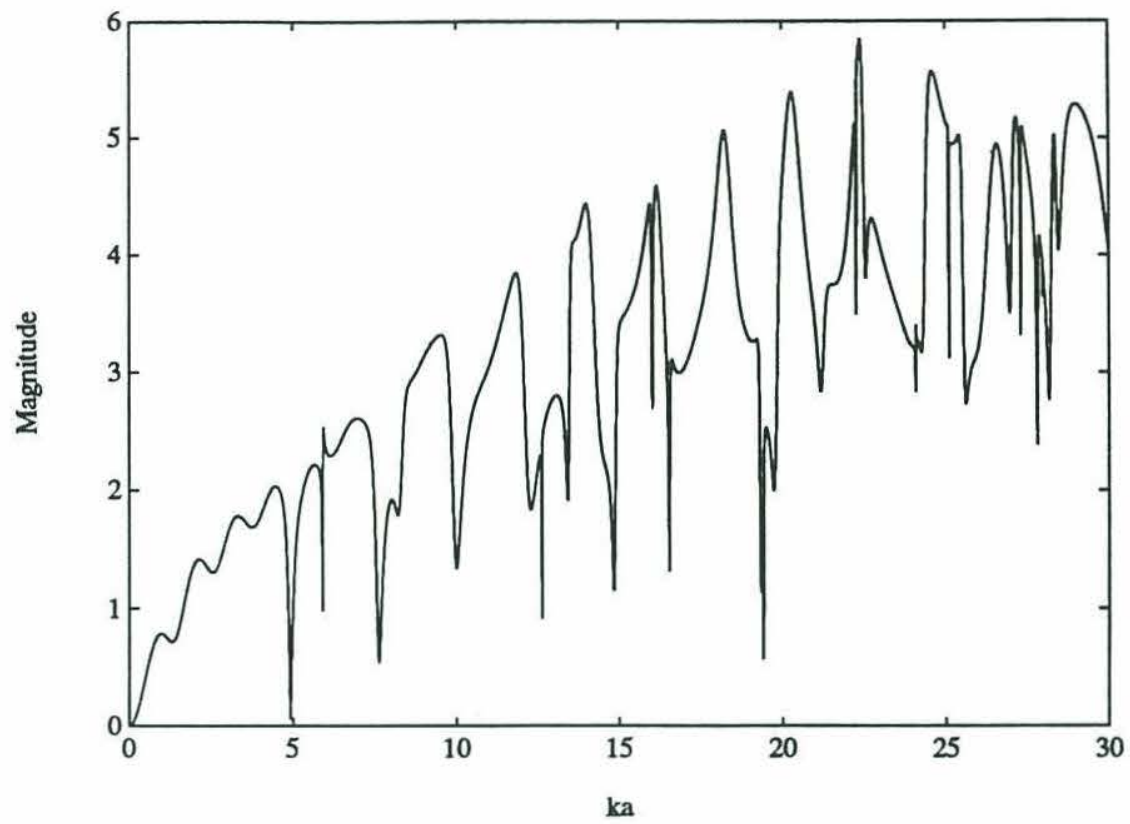

Figure 3-2: Converged modal summation for $0 \leq k a \leq 30$. The summation quickly converges for $m \gtrsim k a$ so the limits of summation were changed from $\sum_{m=0}^{\infty}$ to $\sum_{m=0}^{k a+10}$ for efficient calculation.

ing incident wave and we are assuming the incident field is locally plane at each point on the cylinder, $k a$ is no longer constant in the modal summation term even in the zero roughness case. The value of $k a$ decreases as we move away from $z=0$ and $k a(z)=k a / \sqrt{1+(z / R)^{2}}$ where $\mathrm{R}$ is the source/receiver to target separation (Eq. 2.77). If the summation term were slowly varying, we could consider asymptotic approximations to the integral such as the method of stationary phase [21]. However, Fig. 3-2 shows that the summation term is not analytic at the sharp nulls thus making the asymptotic approximations invalid near these regions. Figure 3-3(a) shows a typical integrand calculated in a slowly varying region away from any nulls. The broad central region corresponds to the first Fresnel zone and the oscillations with increasing $z$ come from the phase shifts in subsequent higher order Fresnel zones. The integrand shows a quick decay due to the Gaussian beam pattern and $z^{-2}$ dependence in the integrand. Since the oscillating tails associated with the higher order Fresnel zones tend to cancel one another, the stationary phase method 
approximates this integral as the area under the broad central maxima, the region due to the first Fresnel zone. Figure 3-3(b) illustrates the behavior of the integrand near a null. For $k a$ values just above a null, the local normal component of $k a$ $\left(k a(z)=k a / \sqrt{1+(z / R)^{2}}\right)$ will match that of the null location at some point in the main section of the integrand destroying the regular Fresnel zone oscillations. The higher Fresnel zones no longer cancel and, as we show, this leads to oscillations in the integrated solution for $k a$ values directly above nulls. Figures 3-3(c) and (d) show the complicated structure of the integrand once when surface roughness is also included.

Since the integral can vary rapidly (Fig. 3-3(d)), an extended trapezoidal integration scheme is necessary to achieve convergence $[43,44]$. The indefinite integral is divided into subintervals, then a coarse approximation to the integral of each subinterval is made using a two step trapezoidal rule

$$
\int_{a}^{b} f(z) d z=\frac{b-a}{2}\left[\frac{1}{2} f(a)+f((a+b) / 2)+\frac{1}{2} f(b)\right]+O\left(\frac{(b-a)^{3} f^{\prime \prime}}{2^{2}}\right)
$$

Now halving the step size, the error in the approximation is decreased by factor of 4 , and the second stage trapezoidal approximation is

$$
\begin{aligned}
\int_{a}^{b} f(z) d z= & \frac{b-a}{4}\left[\frac{1}{2} f(a)+f((3 a+b) / 4)+f((a+b) / 2)+\right. \\
& \left.f((a+3 b) / 4)+\frac{1}{2} f(b)\right]+O\left(\frac{(b-a)^{3} f^{\prime \prime}}{4^{2}}\right)
\end{aligned}
$$

This second stage uses all the information calculated in the first stage and adds two new interior points to refine the approximation. We can iteratively repeat this process, halving the step size and adding $2^{n-1}$ points at the $n$th stage, until the fractional error between subsequent stages (and hence an estimate of the accuracy of the approximation) falls below some bound $\varepsilon_{1}$. This adaptive trapezoidal algorithm iteratively finds the appropriate step size for any given subinterval of the integral and error bound $\varepsilon_{1}$. This provides a robust method of integrating the integrands where the higher order derivatives do not exist (Fig. 3-3(d)) and the appropriate step size is initially unknown. 

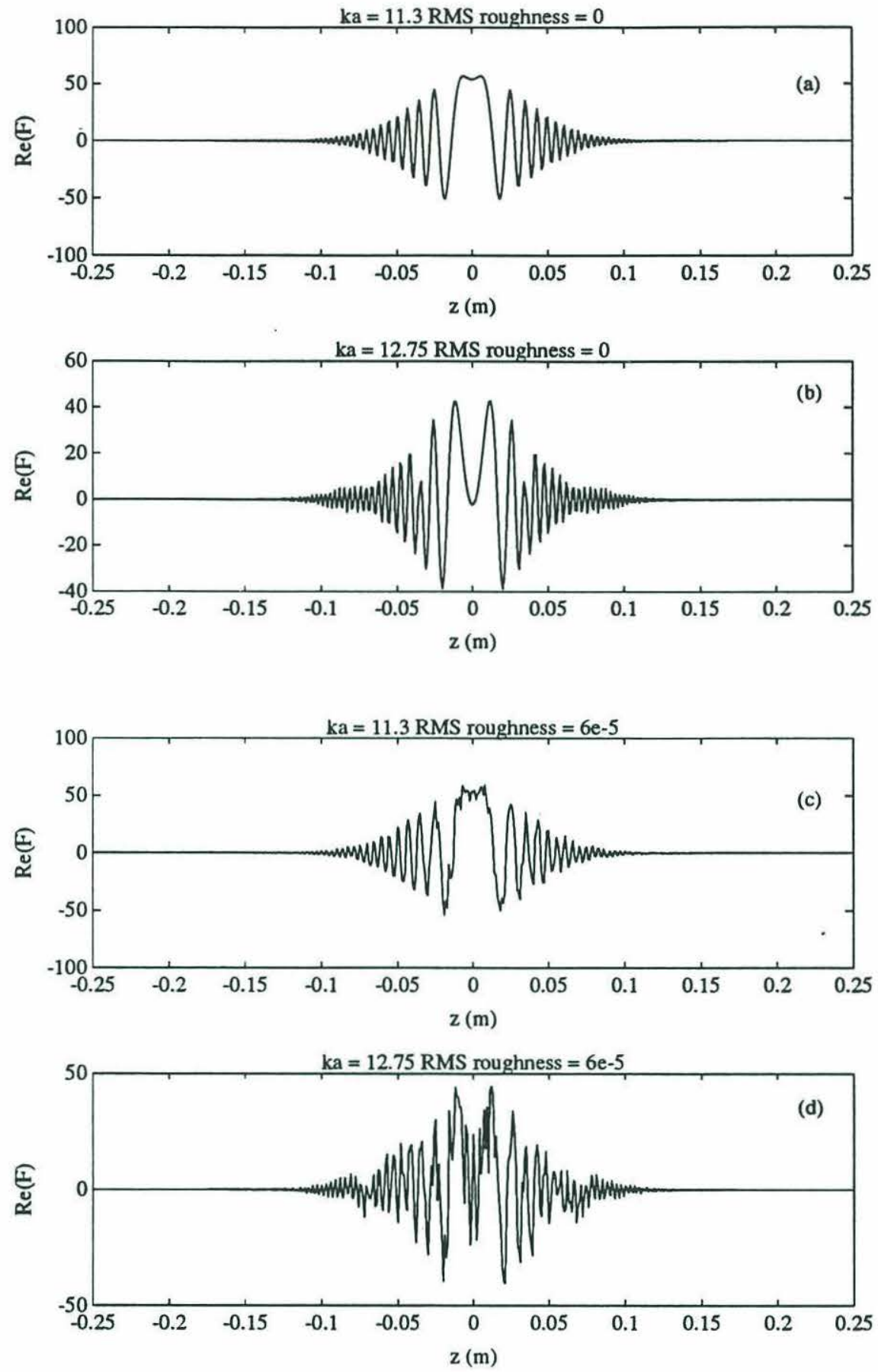

Figure 3-3: Real part of integrand for infinite cylinder. (a) and (b) represent typical smooth cylinder integrands while (c) and (d) correspond to rough cylinder $\left(\sigma_{s}=60 \mu \mathrm{m}\right)$ integrands for the same values of $k a$. The value of $k a(k a=11.3)$ in (a) and (c) is in a slowly varying region of the modal summation, away from any nulls. In (b) and (d), $k a=12.75$ is just above a sharp null in the modal term. The imaginary part of the integrand shows the same structure. 
The algorithm calculates the integral of the central first Fresnel region (the integrand is very smooth and converges quickly with a coarse step size) then begins marching out from the first Fresnel zone calculating new subintervals separately (these require a finer step size due to the oscillations in the integrand). This algorithm continues adding intervals until the total integral converges within some error bound $\varepsilon_{2}$. Since subsequent subintervals have an ever decreasing contribution to the total integral (due to the rolloff seen in Fig. 3-3), the algorithm iterates a few times to estimate the magnitude of the integral for a given interval and then relaxes the trapezoidal error bound $\varepsilon_{1}$ based on the magnitude of that subinterval integral relative to the magnitude of the total integral. Whereas we need to calculate the first Fresnel region (the main contribution to the total integral) to a high accuracy, the contributions to the total integral from a subintervals out in the tails will be several orders of magnitude less than that of the first Fresnel region and there is no need to calculate these "tail" regions to the same order of accuracy.

Although this adaptive trapezoidal scheme is robust, it can be very slow and since the numerical integration scheme must be repeated for each value of $k a$, this leads to inordinately long computation times. Even with surface roughness included, some regions of $k a$ (Figures 3-3(a) - 3-3(c)) appear sufficiently smooth for higher order integration techniques which require higher order derivatives of the integrand to be finite. One method, Romberg integration, closely follows the trapezoidal scheme already developed. At each stage in a trapezoidal scheme, the Romberg method considers all the estimates from the previous $k$ stages as a series and extrapolates the series to its limiting value for a step size of zero. If the integrand is sufficiently smooth, this method will converge must faster with fewer evaluations of the integrand than the strict trapezoidal scheme (Press et al. give a typically example where the Romberg method required $2^{8}$ fewer functional evaluations than a trapezoidal routine for the same accuracy [44]). By saving the previous estimates from the trapezoidal scheme as the step size is reduced, we can parallel 
both methods, stopping the iteration as soon as either method converges. Thus we get the speed of the Romberg method for sufficiently smooth intervals, and the robust convergence of the trapezoidal method for non-analytic regions. Using both methods in parallel decreases computer run times by over a factor of two.

Both integration schemes require many evaluations of the integrand over very small step sizes. This requires converging the modal summation term for many closely spaced values (and in many cases the same value) of $k a$. In turn, each term in the modal summation requires the calculation of several Bessel function to compute $\eta_{m}$ (Eq. 2.48). This creates a bottleneck which limits the processing speed. For a given set of realizations, we first calculated the modal summation term on a fine grid $(\Delta k a=0.01)$ over the entire $k a$ range of interest. These values formed a look up table from which other values could be interpolated. Comparisons between using the exact modal sum and interpolated values in the integral showed small interpolation errors for $k a$ values right at a sharp discontinuity in modal summation ( $k a=12.62)$ but average relative errors of the order $10^{-5}$ for regions away from any sharp nulls or discontinuities. Interpolating the modal summation term further decreases run times by 2 orders of magnitude.

\subsection{Comparison of smooth and rough cylinder so- lutions}

Using the numerical schemes developed in Section 3.2, the scattered field from an infinitely long elastic cylinder with a point source and receiver (Eq. 2.81) can be computed. The input parameters used, which are similar to those in our experiment, are: source/receiver to target separation $R=24 \mathrm{~cm}$; characteristic beamwidth of the source $/$ receiver $\Theta_{o}=26^{\circ}$; sound speed $c$ and density $\rho$ of the fluid, $1477 \mathrm{~m} / \mathrm{sec}$ and $1.0 \mathrm{~g} / \mathrm{cc}$ respectively; and material properties of the elastic cylinder, $c_{l}=5790$ $\mathrm{m} / \mathrm{sec}, c_{s}=3100 \mathrm{~m} / \mathrm{sec}$, and $\rho_{1}=7.9 \mathrm{~g} / \mathrm{cc}$ (values for stainless steel from [45]). 


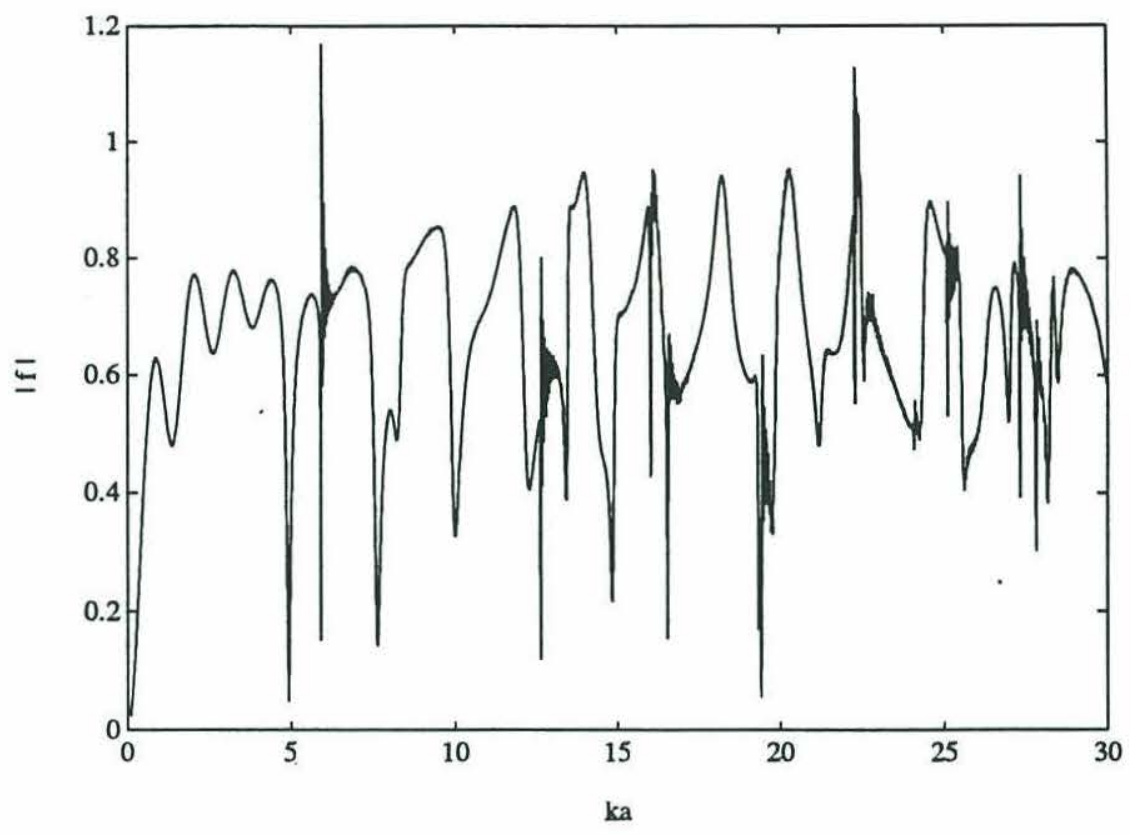

Figure 3-4: Magnitude of the backscattered form function $\left(|f| \equiv\left|f^{\infty}\right|\right.$ from Eq. 2.81) from a smooth infinite-length elastic cylinder for a spherically spreading directional source and receiver with a Gaussian beam pattern. This numeric solution is based on the approximate deformed cylinder formulation. Input variables are sound speed for fresh water $c=1477 \mathrm{~m} / \mathrm{s}$, and the material properties for stainless steel $c_{s}=$ $3100, c_{l}=5790 \mathrm{~m} / \mathrm{s}$, and $\rho_{1}=7.9$. The source distance and beamwidth were $R=$ $24 \mathrm{~cm}$ and $\Theta_{o}=26^{\circ}$. Rapid oscillations at $k a \approx 6,12,16,19$, etc. are due to the incomplete cancellation of higher order Fresnel zones near sharp modal nulls due to the spherically spreading incident field.

Figure 3-4 shows oscillations in the scattered field for $k a$ values directly above sharp discontinuities in the modal summation. These oscillations are due to incomplete cancellation of the higher order Fresnel zones when $k a \cos \theta$ matches the modal null $k a$ as shown in Figures 3-3(b) and (d).

This numerical scheme can also be used to perform Monte Carlo simulations for rough cylinder scattering. Multiple independent random profiles can be computed from Eq. (3.2) using the same surface statistics, and the scattered field from each of these realizations can numerically integrated. Figures 3-5 - 3-8 show the fluctuations in the scattered field for 150 simulated cylinders at different values of the 


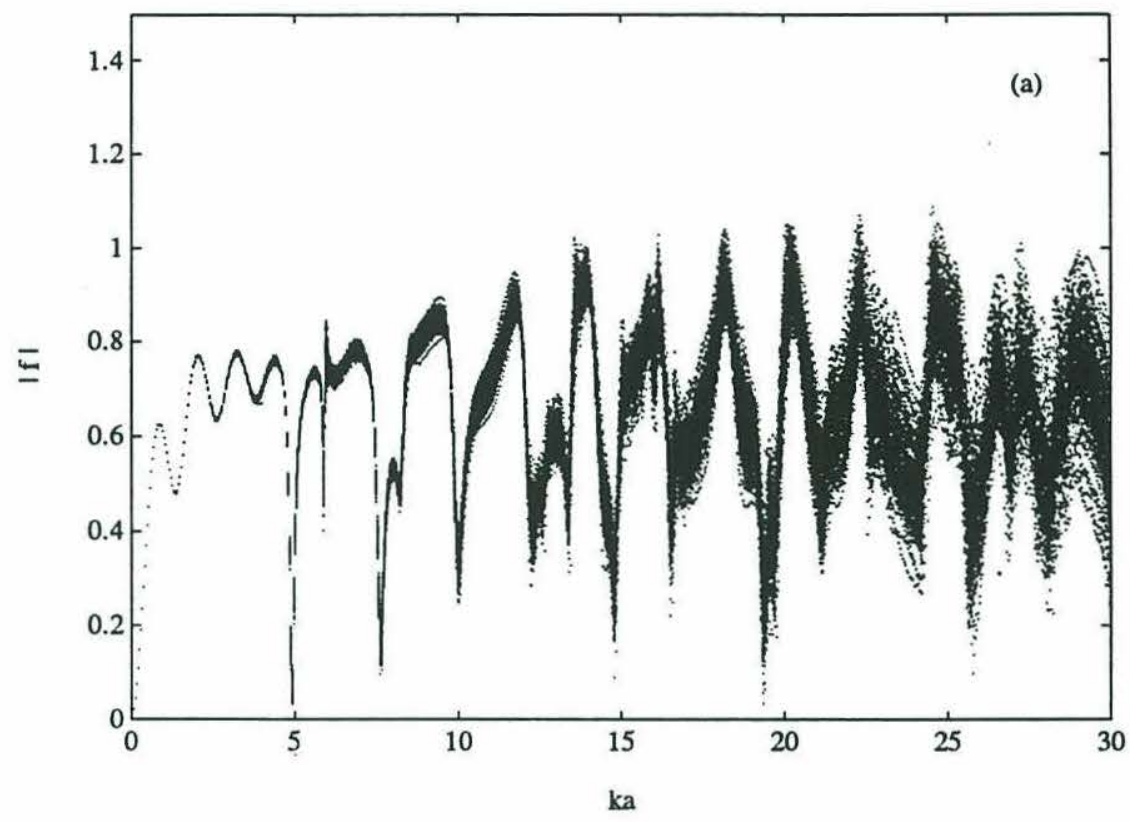

Figure 3-5: Backscattering from randomly rough infinite-length elastic cylinders for a spherically spreading directional source and receiver with a Gaussian beam pattern and fractional roughness $\sigma_{s} / a=0.005$. The scatter plot superimposes field solutions for 150 simulated realizations. The elastic material properties are the same as Figure 3-4 and the surface roughness bandlimits are $K_{\text {low }}=169 \mathrm{~m}^{-1}$ and $K_{\text {high }}=2934 \mathrm{~m}^{-1}$. As $k a$ increases, variability is shown to increase indicating increased effects due to roughness.

fractional roughness $\sigma_{s} / a$. The relative fluctuations in the field are dependent upon the relative roughness $k \sigma_{s}$ with a theoretical transition between the low and high fluctuation regions at $k \sigma_{s} \approx 0.5[17,18]$. This transition point occurs at $k a=25$ in Fig. 3-7 and $k a=12.5$ in Fig. 3-8. Taking an ensemble average over the simulation at each value of $k a$, the mean and mean square fields (Eqs. 2.82 and 2.83) can be found from the same numerical data (Fig. 3-9). Figure 3-9 shows a general decrease the mean field with increasing roughness. Stanton [17] shows that the specular and higher order $(m>0)$ Rayleigh waves are attenuated at a much higher rate than the $m=0$ Rayleigh wave due to one-dimensional surface roughness. This causes the rolloff in the mean field level and the decrease in the mean field oscillations seen in Fig. 3-9. 


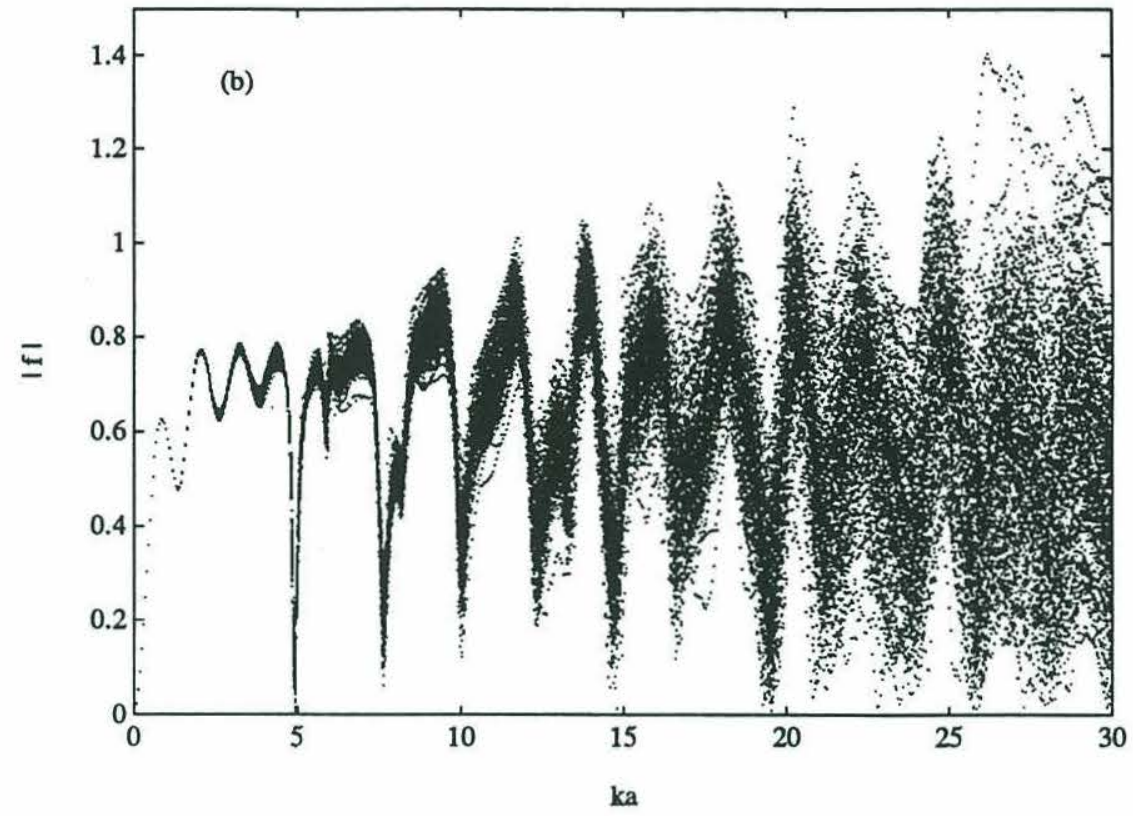

Figure 3-6: Backscattering from randomly rough infinite-length elastic cylinders with fractional roughness $\sigma_{s} / a=0.0131$. See Figure 3-5 for details.

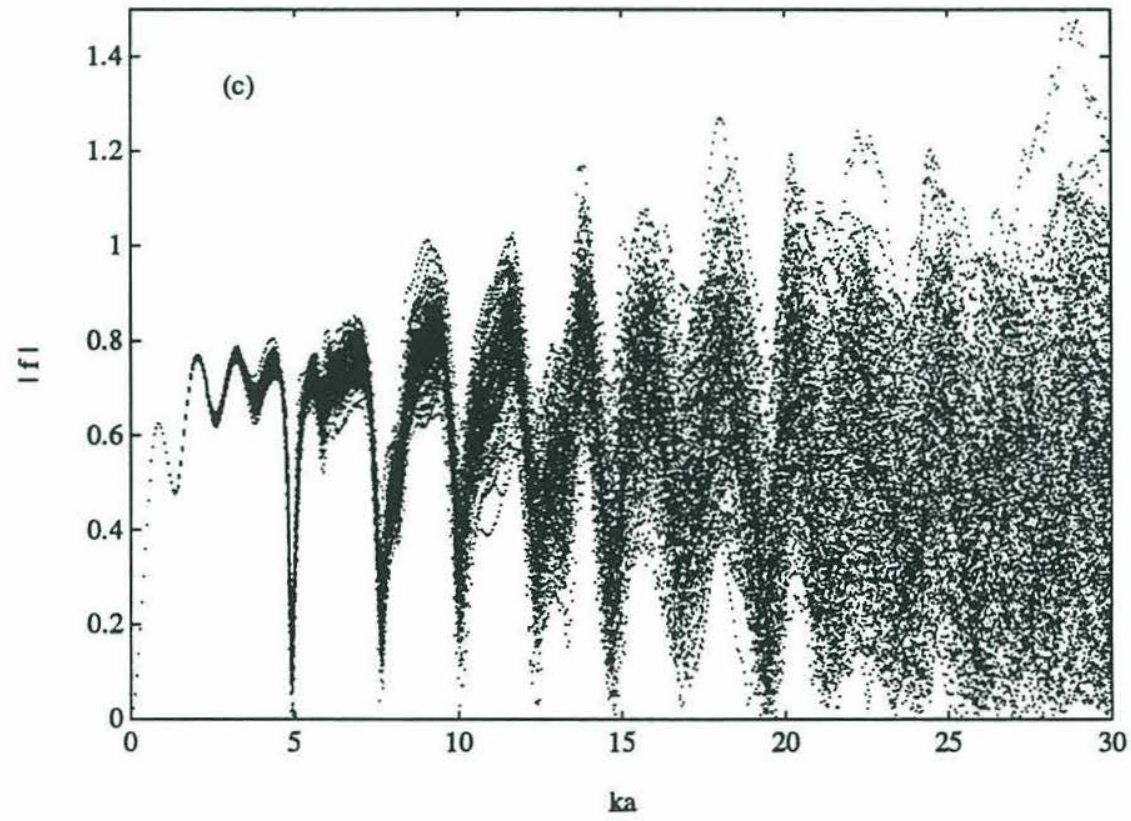

Figure 3-7: Backscattering from randomly rough infinite-length elastic cylinders with fractional roughness $\sigma_{s} / a=0.02$. See Figure 3-5 for details. 


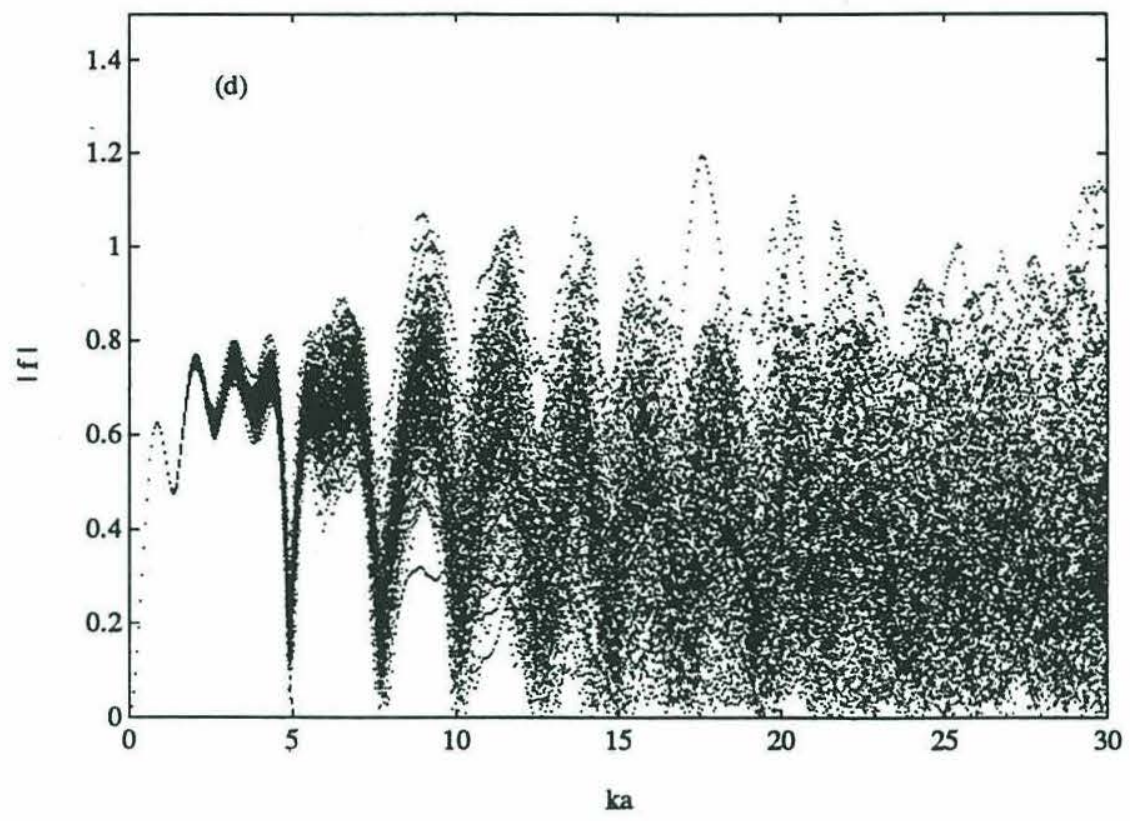

Figure 3-8: Backscattering from randomly rough infinite-length elastic cylinders with fractional roughness $\sigma_{s} / a=0.04$. See Figure 3-5 for details.
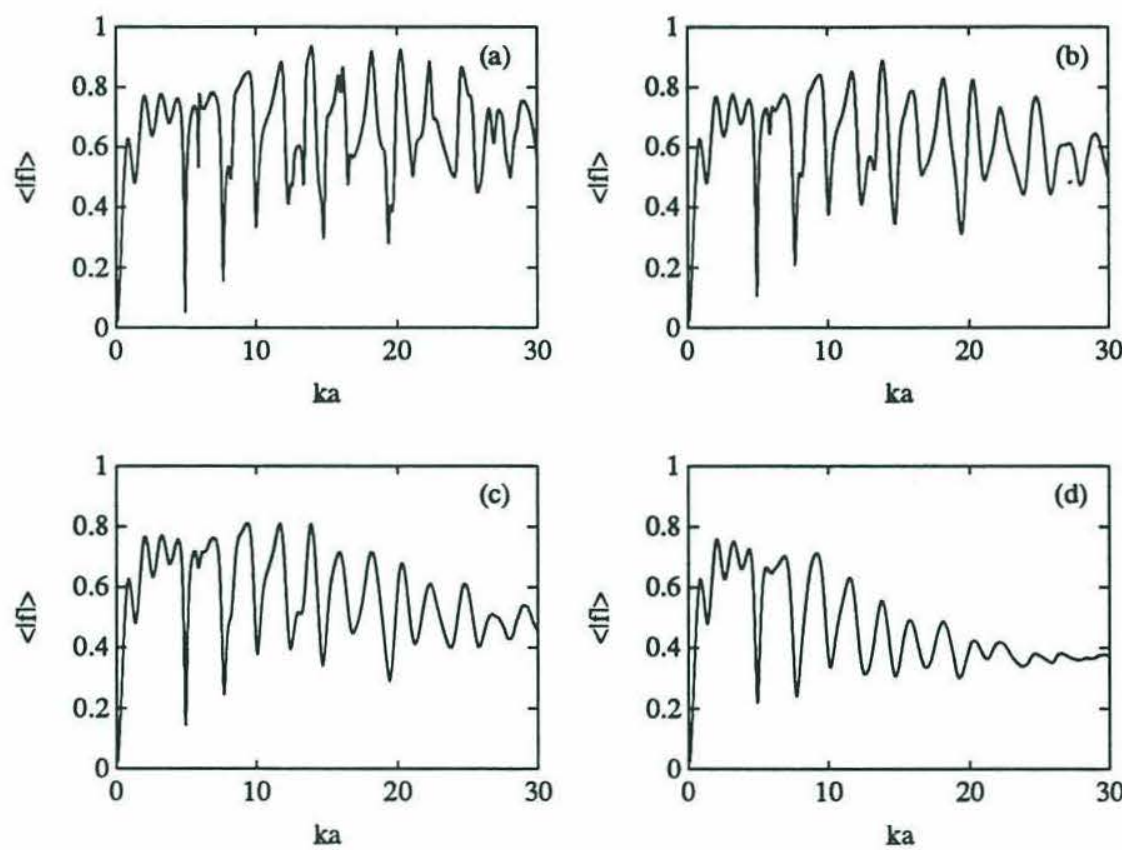

Figure 3-9: Mean backscattered field from randomly rough infinite-length elastic cylinders for various values of fractional roughness $\sigma_{s} / a$. Solutions for mean field calculated from an ensemble average of 300 simulated realizations. (a) is mean of data in Fig. 3-5, (b) Fig. 3-6, (c) Fig. 3-7, and (d) Fig. 3-8. 
Analyzing the variance, $\sigma_{|f \infty|}^{2}$, of the fluctuations for a given value of fractional roughness (Fig. 3-10) shows a clear increase in field fluctuations with increasing relative roughness, $k \sigma_{s}$, and a transition to "saturation" (Rayleigh-like distribution) at $k \sigma_{s} \approx 0.5$. The theoretical Rice PDF shape parameter, $\gamma$, can be estimated from the field statistics for low fluctuations [18]

$$
\gamma=\frac{\left\langle\left|f^{\infty}\right|^{2}\right\rangle}{2 \sigma_{|f \infty|}^{2}} .
$$

However for $\gamma<10$, this low roughness approximation is not valid and a least square fit was performed, comparing the fluctuation distribution to the Rice PDF while varying $\gamma$ from 0 to 10 in increments of 0.1 . Histograms of the scattered field show that the Rice PDF provides a good approximation for the fluctuation distribution at $k a$ values away from modal nulls over the entire roughness scale (Fig. 3-11). At low $k a\left(k \sigma_{s} \ll 1\right)$ the fluctuations are small and the histograms have a narrow Gaussian-like character $(\gamma \gg 1)$. As $k a$ (and $k \sigma_{s}$ ) increase, the surface becomes acoustically rougher and the fluctuations in the field increase. The PDFs broaden and approach a Rayleigh curve $(\gamma=0)$. Figure 3-11 is indicative of the close fit between the Rice PDF and the fluctuation distributions over most $k a$ values.

For $k a$ values near a null, one would expect a bimodal distribution of the fluctuations due to the discontinuity in the modal summation. Some realizations would represent field fluctuations about a mean $\left\langle\left|f^{\infty}\right|\right\rangle$ for a $k a$ on one side of the discontinuity whereas realizations with $k a$ on the other side of the discontinuity would fluctuate about a different $\left\langle\left|f^{\infty}\right|\right\rangle$. This should result in a non-Ricean bimodal PDF. Stanton and Chu [18] note some skewing in fluctuation histograms for $k a$ near nulls. We see similar results in the numerical simulations but no clear bimodal pattern.

Figure 3-12 summarizes the changing shape of the field PDF showing $\gamma$ calculated for every value of $k a$ in the numerical solution. The figure shows the strong dependence $\gamma$ on $k$ (trend of $\left.\gamma \propto k^{-4}\right)$ in the region of low roughness $(\gamma \rightarrow \infty)$. In the region of high roughness $(k \sigma \geq 0.5)$, the fluctuations are large and $\gamma(\sim 0)$ is essentially independent of $k$. 

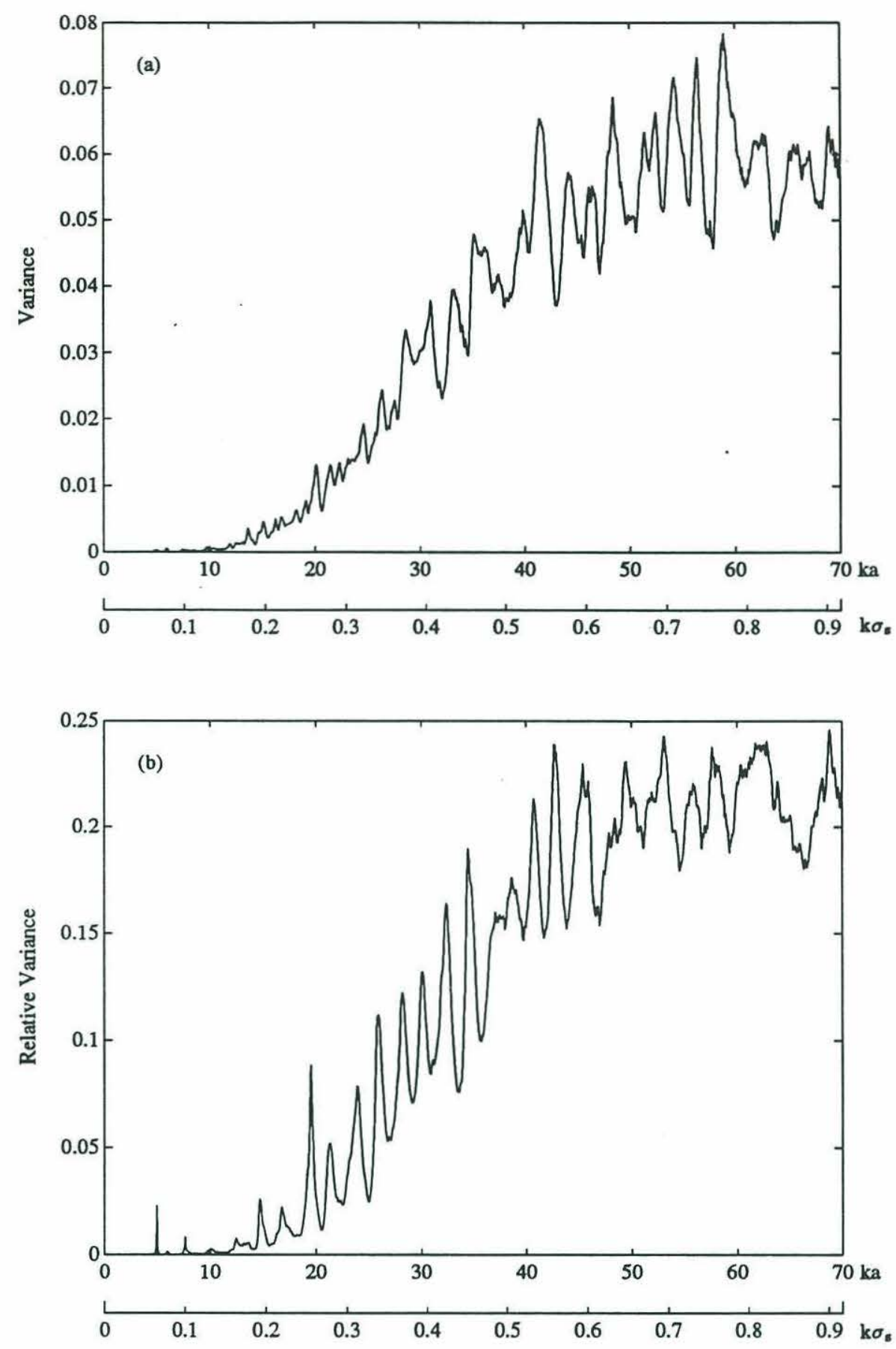

Figure 3-10: (a) Variance $\left(\sigma_{|f \infty|}^{2}\right)$ and (b) relative variance $\left(\sigma_{|f \infty|}^{2} /\left\langle\left|f^{\infty}\right|^{2}\right\rangle\right)$ for backscattered field from randomly rough cylinders. Numerical simulation based on 300 realizations for $\sigma_{s} / a=0.0131$. 

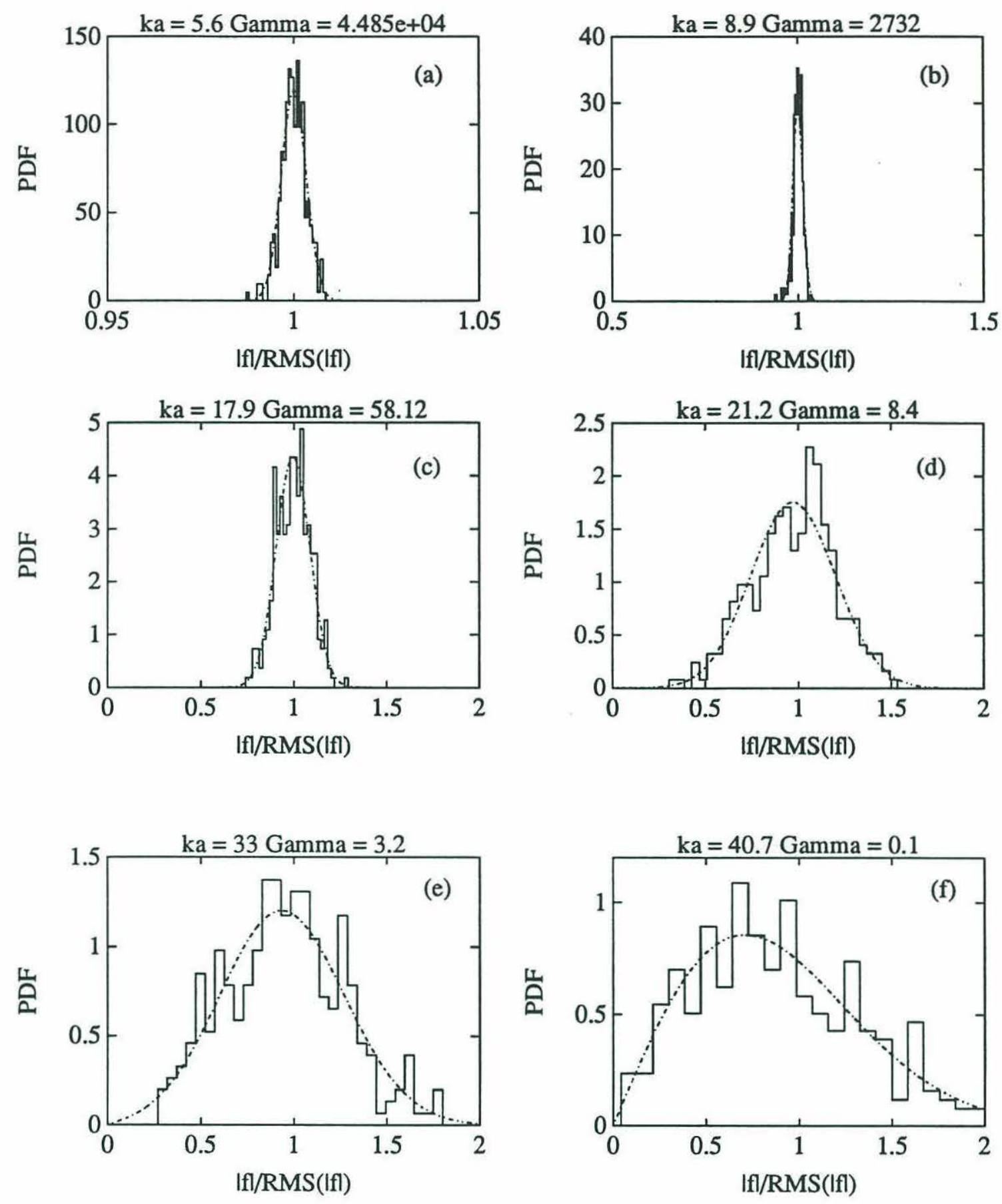

Figure 3-11: Histograms of normalized scattering amplitude $\left|f^{\infty}\right| /\left\langle\left|f^{\infty}\right|^{2}\right\rangle^{1 / 2}$ from 300 simulated realizations (Fig. 3-10) for various $k a$ values. Rice PDFs are superimposed with shape parameter $\gamma$ calculated from the fluctuations of $\left|f^{\infty}\right|$. 


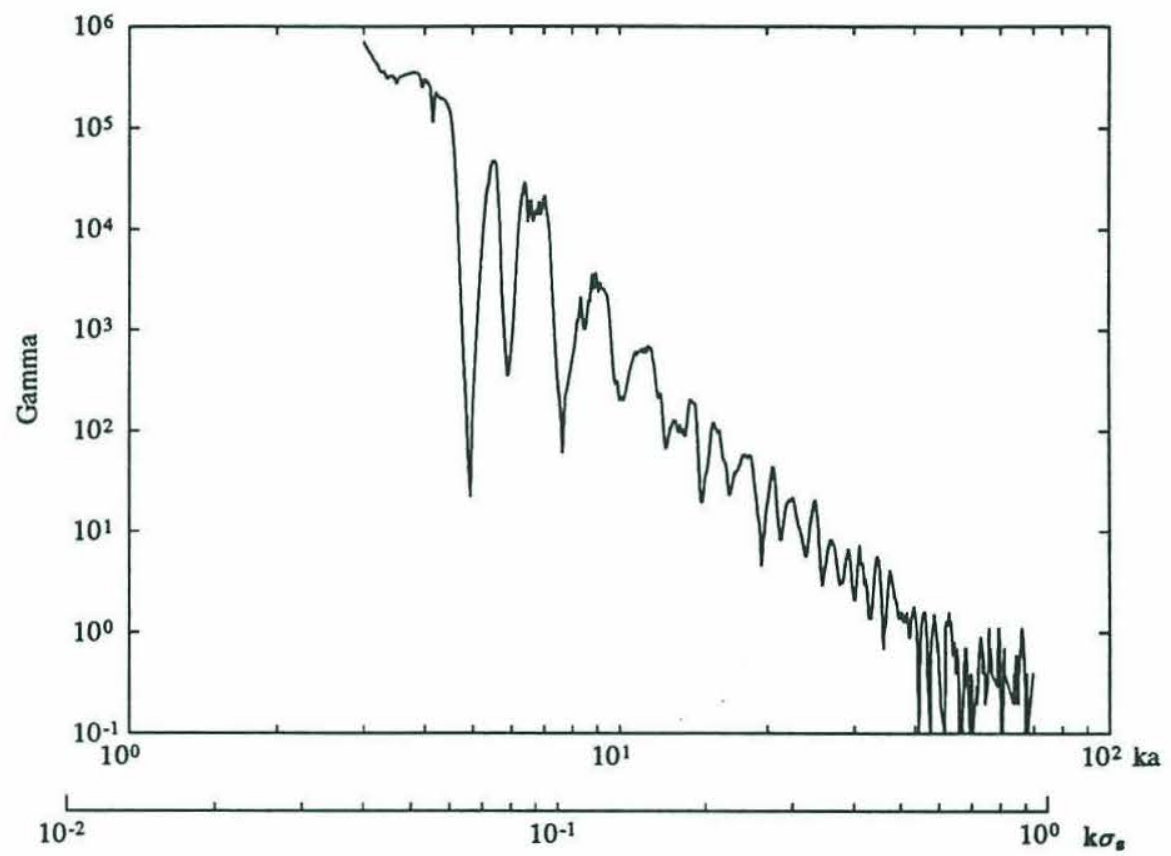

Figure 3-12: Rice PDF shape parameter $\gamma$ plotted against $k a$ and $k \sigma_{s} .300$ numerical realizations per $k a$ were used to calculate $\gamma$. The figure illustrates the rapid decrease in $\gamma$ as $k$ increases and the transition to saturation at $k \sigma_{s} \approx 0.5$. 


\section{Chapter 4}

\section{Experimental Measurements}

A series of experiments were designed to quantitatively measure the roughness related effects in the scattered field discussed in Chapters 2 and 3. While there is a large body of published data for rough planar surface scattering with application to ocean bottom or sea surface problems, only recently has attention been given to the more general problem of rough three dimensional objects. Furthermore, in much of the published rough surface data, the surface profile and statistics are not quantitatively known a priori. In the experiments described here, an exact surface profile was designed based on the mathematical models in Section 3.1 and then precisely manufactured. Thus accurate comparisons can be made between the measured scattered field and analytical expressions using the theoretical formulations developed in Section 2.1.5 with the known surface profile.

\subsection{Target design and manufacture}

A rough cylinder target was manufactured from six $3 / 8$ " 303 stainless steel stock cylinders. Previous investigators $[31,32]$ have used tungsten carbide as an elastic medium since it is very dense $\left(\rho=14 \mathrm{~g} / \mathrm{cm}^{3}\right)$ and thus transmitted bulk waves are insignificant over moderate $k a$ ranges $(k a<100)$. This allows a simplified 


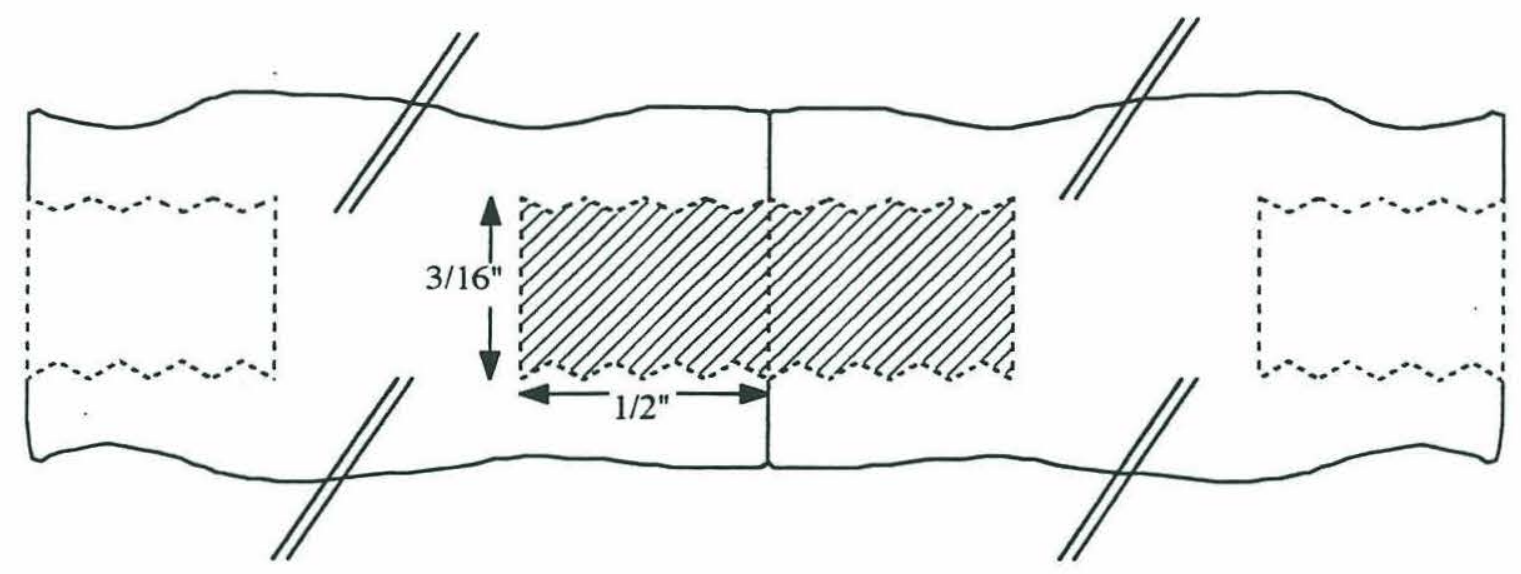

Figure 4-1: Cutaway diagram showing target and threaded pin joining different subsections. Each rough subsection is $\approx 15 \mathrm{~cm}$ long and has a mean diameter $0.9126 \mathrm{~cm}$. Threaded pins $0.47625 \mathrm{~cm}$ in diameter and $1.27 \mathrm{~cm}$ long were used to screw the pieces together.

ray solution (Section 2.1.3) interpretation in terms of the dominate specular and Rayleigh components only. Stainless steel was chosen for these experiments since it is machinable and also relatively dense $\left(\rho=7.9 \mathrm{~g} / \mathrm{cm}^{3}\right)$. It would have been very difficult to precisely lathe a series of tungsten carbide targets. Each subsection had a $3 / 16 " \times 1 / 2$ " hole drilled and tapped in each end and threaded pins made from the same 303 stainless steel were then used to screw the subsections together (Figures 4-1 and 4-2). Care was taken during manufacture to minimize any possible voids between the ends of the pins and the bottoms of the tapped holes. The sections were assembled underwater to avoid internal air bubbles and thus approximate one continuous isotropic elastic cylinder. Two 8" 303 stainless steel cylinders were machined smooth to the same radius as the rough cylinder mean radius. Each was drilled and tapped on one end only giving a smooth cylinder "standard" target $40.64 \mathrm{~cm}$ long.

The target perturbation profile $\varsigma(z)$ was generated using Eq. (3.2) where the 
bandlimits for the surface were based on the first Fresnel zone diameter. The experiment was initially designed based on a source/receiver to target separation of $70 \mathrm{~cm}$ and a frequency range of $350 \mathrm{kHz}$ to $3.5 \mathrm{MHz}$. Assuming a sound speed in water of $c=1500 \mathrm{~m} / \mathrm{s}$, the first Fresnel zone (Eq. 2.78) ranges from $7.75 \mathrm{~cm}$ at $350 \mathrm{kHz}$ to $2.5 \mathrm{~cm}$ at $3.5 \mathrm{MHz}$. Using the criteria for the surface bandlimits derived in Chapter 3 (Eq. 3.4), the design bandlimits for the target surface were $\mathcal{K}_{\text {low }}=811 \mathrm{~m}^{-1}$ and $\mathcal{K}_{\text {high }}=2565 \mathrm{~m}^{-1} ;$ and the input RMS roughness, $\sigma_{s}$, was 60 $\mu \mathrm{m}$.

The perturbation $\zeta(z)$ was calculated at intervals of $0.01 \mathrm{~cm}$ over an entire meter using the above parameters. This profile was then divided into six sections, each approximately $15 \mathrm{~cm}$ long, such that the dividing points between sections corresponded to zero crossing points in the perturbation profile. A mean radius value $a_{o}=0.45625 \mathrm{~cm}$ was added to the perturbations giving a radius profile

$$
a_{R C}(z)=a_{\circ}+\varsigma(z)
$$

These profiles were then formatted and transferred to a computer-controlled lathe and the surface profiles were cut into the six cylinders (Fig. 4-2). Due to memory restrictions in the milling machine, each file had to be compressed prior to downloading. Using an adaptive downsampling algorithm, regions in the profile where the surface had a slowly varying slope were sampled coarsely (high degree of downsampling based on local "flatness") whereas regions where the slope varied rapidly were sampled at a much finer interval (little or no downsampling). This generated files where the surface no longer had a regular sampling period $d z$ but rather was defined by a series of points $\left(z, a_{R C}(z)\right)$. These files were converted to inches, radius values multiplied by 2 to give diameter, and rounded to the nearest $1 / 10000$ " (the tolerance of the lathe). After this conversion and rounding the specified mean diameter was .3593 " giving a final mean radius of $0.456311 \mathrm{~cm}$. Each of these files were loaded into a computer-controlled lathe which linearly interpolated between the data points as it milled the specified surface. 


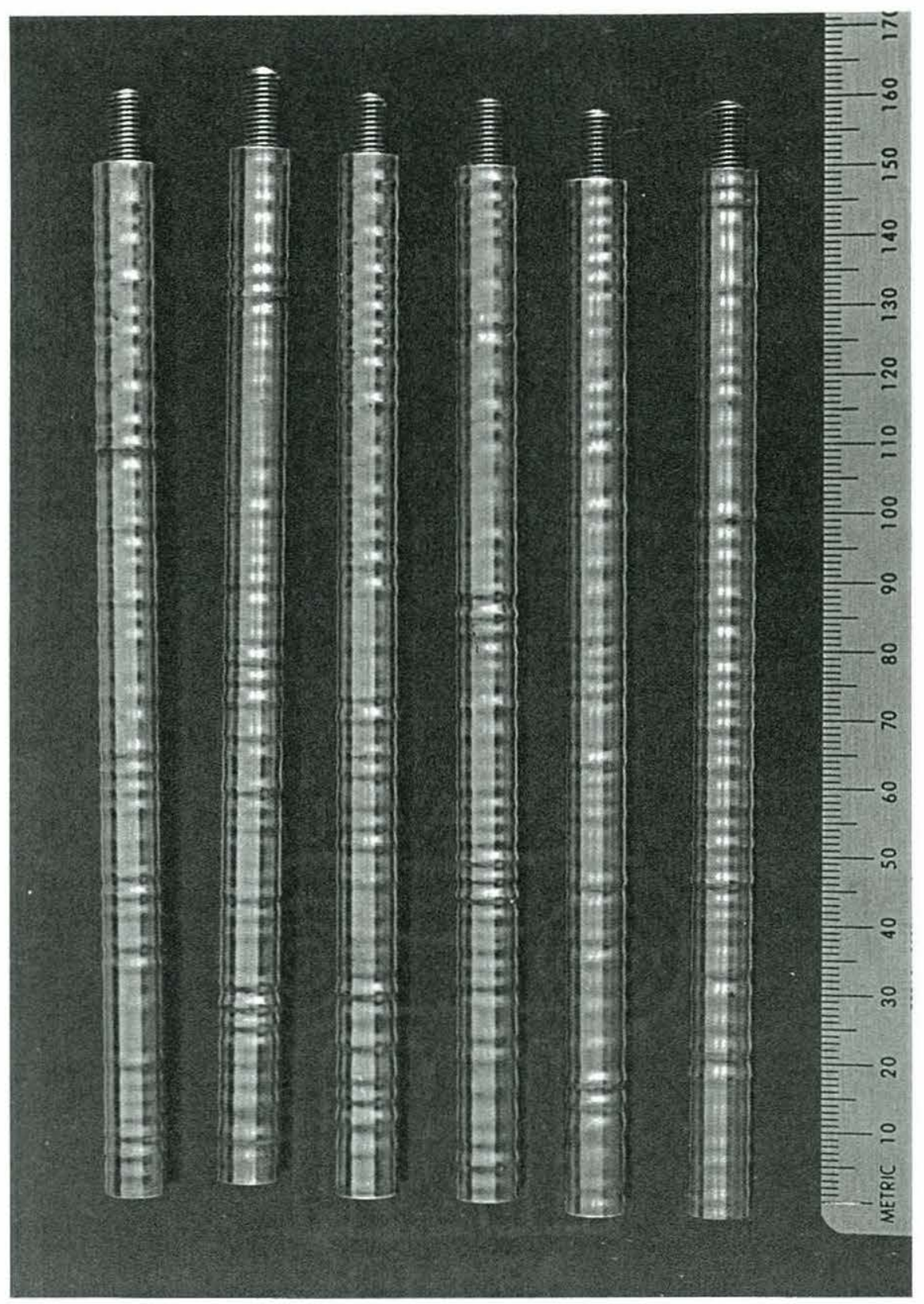

Figure 4-2: Six rough cylinder subsections comprising randomly rough target shown to scale. 


\begin{tabular}{||c|r|r||}
\hline \hline Section & \multicolumn{1}{|c|}{ Mean $(\mathrm{m})$} & Standard deviation $(\mathrm{m})$ \\
\hline \hline Section 1 & $-6.38 \times 10^{-7}$ & $5.87 \times 10^{-5}$ \\
\hline Section 2 & $-1.295 \times 10^{-6}$ & $5.99 \times 10^{-5}$ \\
\hline Section 3 & $1.34 \times 10^{-8}$ & $5.87 \times 10^{-5}$ \\
\hline Section 4 & $-6.91 \times 10^{-7}$ & $6.05 \times 10^{-5}$ \\
\hline Section 5 & $-1.07 \times 10^{-6}$ & $6.01 \times 10^{-5}$ \\
\hline Section 6 & $-5.37 \times 10^{-7}$ & $5.64 \times 10^{-5}$ \\
\hline Total surface & $-7.0 \times 10^{-7}$ & $5.90 \times 10^{-5}$ \\
\hline \hline
\end{tabular}

\begin{tabular}{||l|r|l||}
\hline \hline Design specs & 0.0 & $6.0 \times 10^{-5}$ \\
\hline \hline
\end{tabular}

Table 4.1: Rough cylinder target surface statistics. The sample means and standard deviations are based on reconstructed profiles from the actual lathe control files. The total surface values are based on concatenating the sections end to end. The design specs are from the input values to the numerical routine that generated the surface profile.

In order to check for any errors due to quantization or downsampling artifacts, the surface statistics were analyzed from these machine specification files rather than the original perturbation profile. Each file was converted back to a radius vs. $z$ listing in $\mathrm{cm}$. Then a regular sampling period of $0.01 \mathrm{~cm}$ was restored by linearly interpolating between the existing points. Finally a mean radius of $0.456311 \mathrm{~cm}$ was subtracted from all the points returning to a perturbation profile. Table 4.1 lists the first and second order statistics for the six target subsections and the overall target made by concatenating all six subsections end to end. The small bias in the mean values is due to rounding errors. The original design mean diameter was $0.9125 \mathrm{~cm}$ or $0.359252 "$. After rounding to the machine tolerance, the diameter at the zero crossing points in the perturbation profile became $0.3593^{\text {" }}$ or $0.91262 \mathrm{~cm}$ and this value is used for the mean diameter for the remainder of this work. This error is only 1.5 parts in 10000 . Figure 4-3 shows histogram plots of the radius perturbation for each of the target sections illustrating the Gaussian nature of the actual surface. Superimposed on each histogram is a Gaussian PDF with standard deviation from Table 4.1. The amplitude for each PDF was fitted by normalizing 
the area under the histogram to 1 .

Micrometer reading were taken at numerous locations on the rough cylinders and compared with the design profile. The mean error in diameter over all measurements was $-6.8 \mu \mathrm{m}$ and the standard deviation was $37 \mu \mathrm{m}$. This is within the instrument error of the micrometer $\pm 12.7 \mu \mathrm{m}$. An optical comparator was used to compare of the target profile "waveform" with the design profile. These qualitative checks also verified the actual target profile matched the design profile.

An estimate of the surface profile power density spectrum was made using the maximum entropy method (MEM) algorithm [44,46]. This method provides better resolution than traditional Welch or periodogram methods [47] due to the narrow bandlimited nature of the surface spectrum. For an ideal bandlimited white noise process, the power spectrum is perfectly flat within the bandlimits with a sharp cutoff at the band edges and the process sample are completely uncorrelated. Figure 4-4 shows that the actual power spectrum is fairly uniform within the bandlimits and has a strong roll-off at the design band edges $\mathcal{K}_{\text {low }}=811 \mathrm{~m}^{-1}$ and $K_{\text {high }}=2565 \mathrm{~m}^{-1}$. The spectral peak at $K=1100 \mathrm{~m}^{-1}$ was caused by a bias in the random number generator used in routine that calculated the original perturbation surface. After the target was manufactured, it was discovered that the 100 random numbers used to randomize the wavenumber values in Eq. (3.2) were not uniformly distributed between 0 and 1 but contained a small correlation peak around 0.165 . This resulted in a preferential weighting toward $K=1100 \mathrm{~m}^{-1}$ in the target profile. Even with this spectral weighting artifact, Fig. 4-4 shows that the target surface spectrum is still fairly flat over the band of interest and a good approximation to a bandlimited white process. The surface autocorrelation function $\Phi_{z z}$ (Fig. 4-5) also shows that the surface quickly decorrelates as one expects in a white process. The strong negative correlation around the central peak at $\tau=0$ lag is believed to be related to the spectral peak discussed above and the fact that the surface is slowly varying rather than completely decorrelating between neighboring points as in a true white 

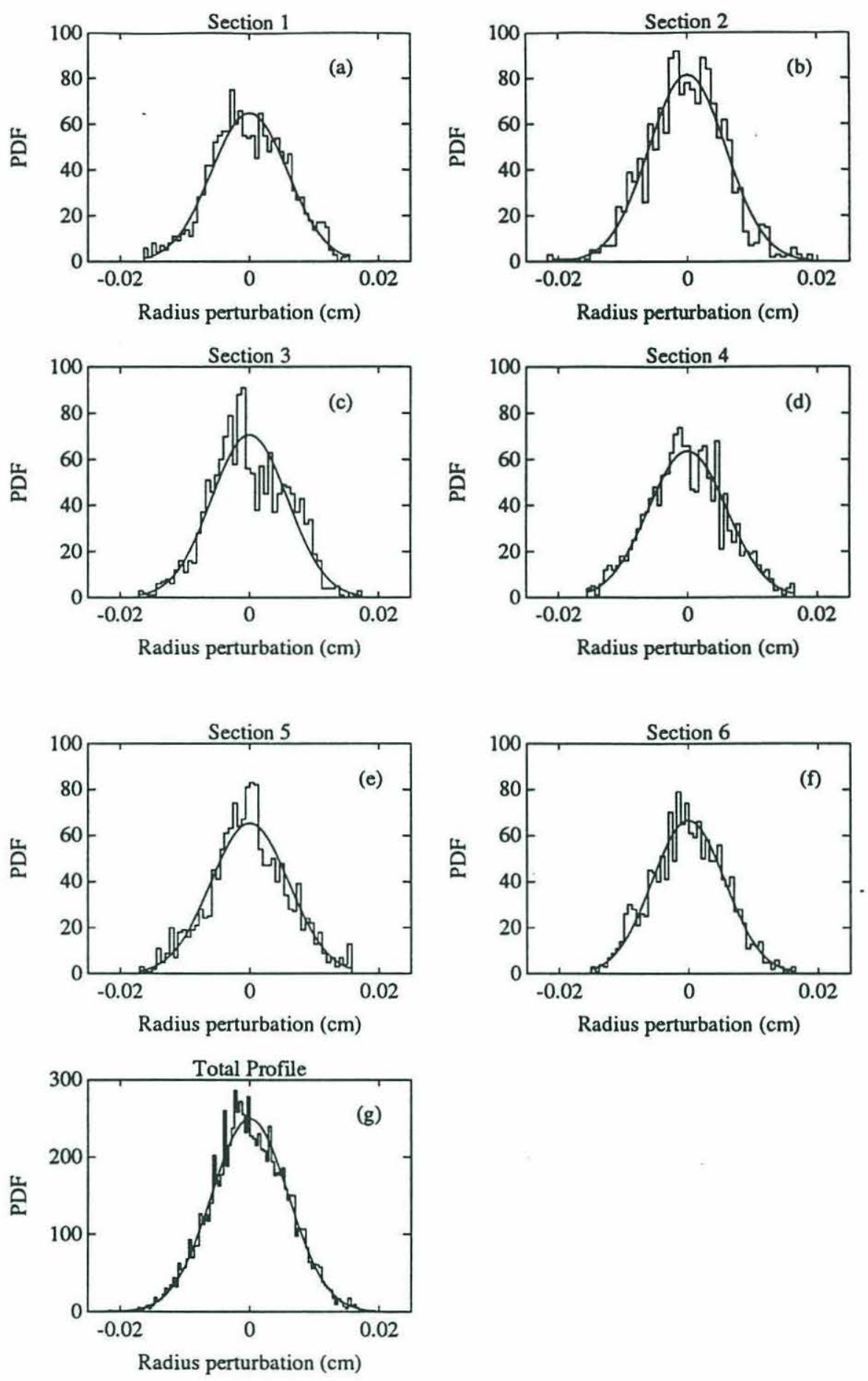

Figure 4-3: Histogram of radius for each of six rough surface subsections (a) - (f) and entire composite target profile ( $\mathrm{g}$ ). A Gaussian PDF has been superimposed on each plot highlighting the Gaussian nature of the profile statistics. 


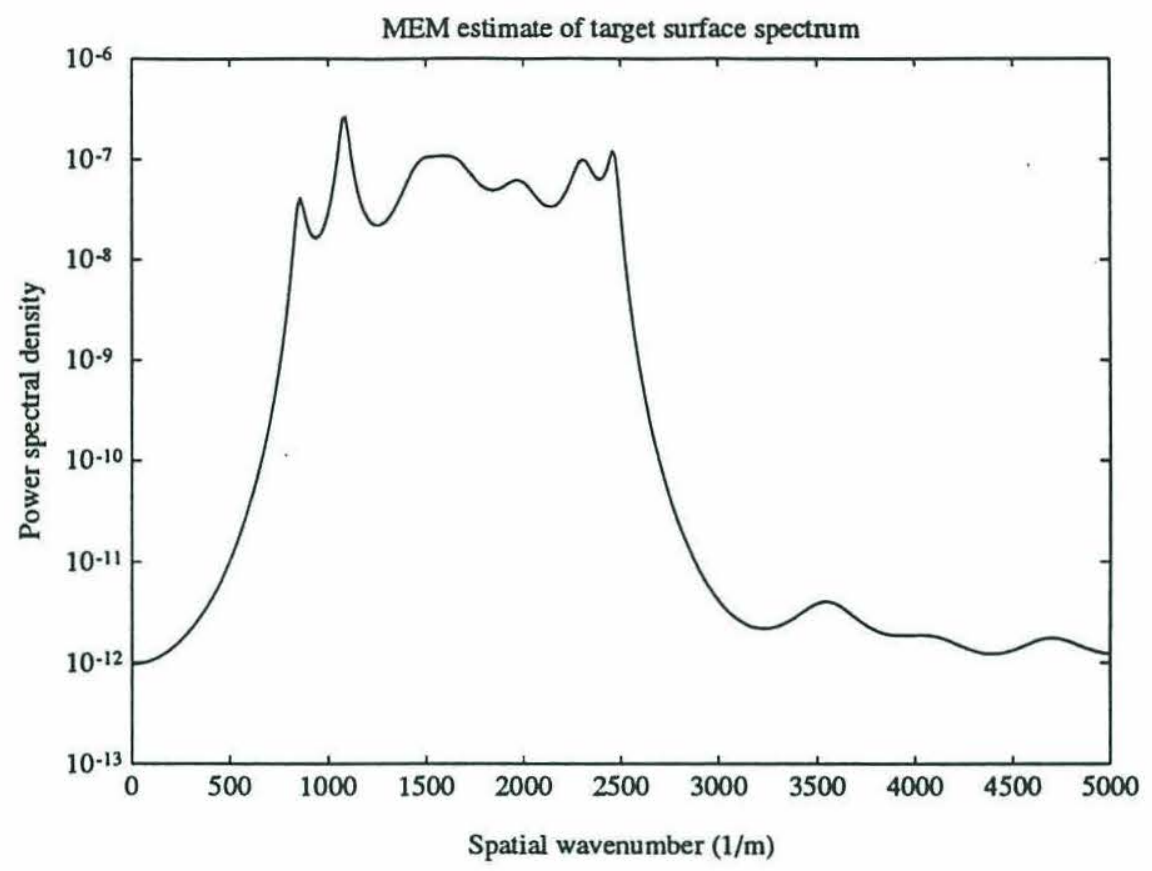

Figure 4-4: MEM estimate of the surface power density spectrum. The spectrum shows the good approximation between the actual surface and a bandlimited white process. The design band edges are $K_{\text {low }}=811 \mathrm{~m}^{-1}$ and $K_{\text {high }}=2565 \mathrm{~m}^{-1}$.

noise process.

Due to physical constraints in the ultrasonic test tank used, the data was collected with a transducer/target separation of $\mathrm{R}=24 \mathrm{~cm}$, quite closer than the $70 \mathrm{~cm}$ originally planned. This effects the ratio of the surface wavelengths to the Fresnel zone size. At $24 \mathrm{~cm}$ the upper bandlimit $K_{\text {high }}$ should have been $4380 \mathrm{~m}^{-1}$ using the $10 \Lambda$ criteria. Even though the actual target bandlimit $K_{\text {high }}=2565 \mathrm{~m}^{-1}$ is too low by a factor of 2 , it still provides 5.9 surface wavelengths over the width of the first Fresnel zone at $3.5 \mathrm{MHz}$ and the surface remains acoustically rough over the entire frequency range. 


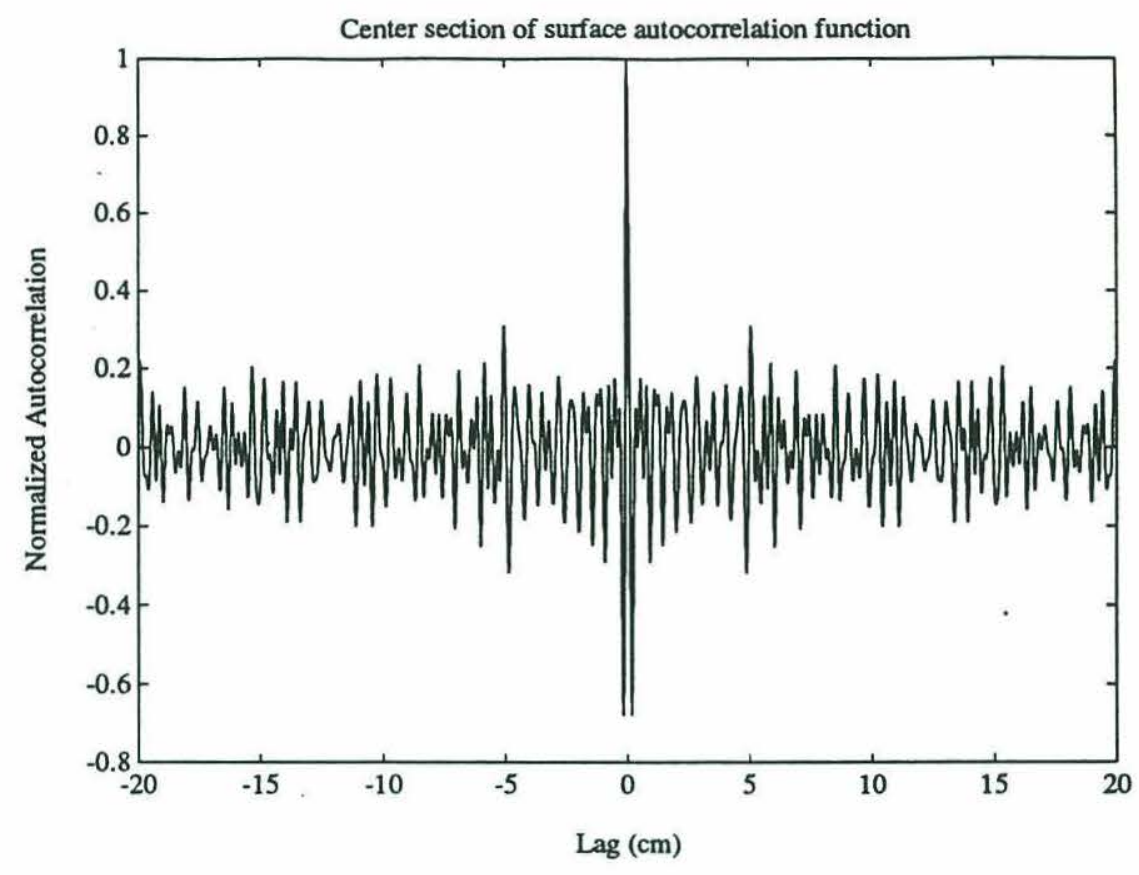

Figure 4-5: Autocorrelation $\left(\Phi_{z z}\right)$ function for the target profile (only center $20 \mathrm{~cm}$ of lag $\tau$ shown).

\subsection{Experimental set-up and alignment}

The experiment was conducted in a $1 \mathrm{~m} \mathrm{x} 1 \mathrm{~m} \mathrm{x} 0.5 \mathrm{~m}$ ultrasonic test tank at the Earth Resources Laboratory of M.I.T. This tank contained alignment apparatus necessary in conducting this experiment. Two closely spaced transducers were used, one as transmitter and one as receiver, approximating a monostatic configuration. Both transducers were identical 1" diameter Krautkramer Branson transducers with a center frequency of $2.25 \mathrm{MHz}$. Due to the inherent damping in the ceramic and backing material, they provided (by design) a continuous broadband response from approximately $300 \mathrm{kHz}$ to $3.5 \mathrm{MHz}$. At these high frequencies, typical flat piston transducers have a very narrow beam. In order to create a wider beam, these transducers were custom made; they were shaped as spherical caps increasing the beamwidth to about 20 degrees $[36,48]$.

The ultrasonic test tank was equipped with two computer-controlled 3-axis mo- 


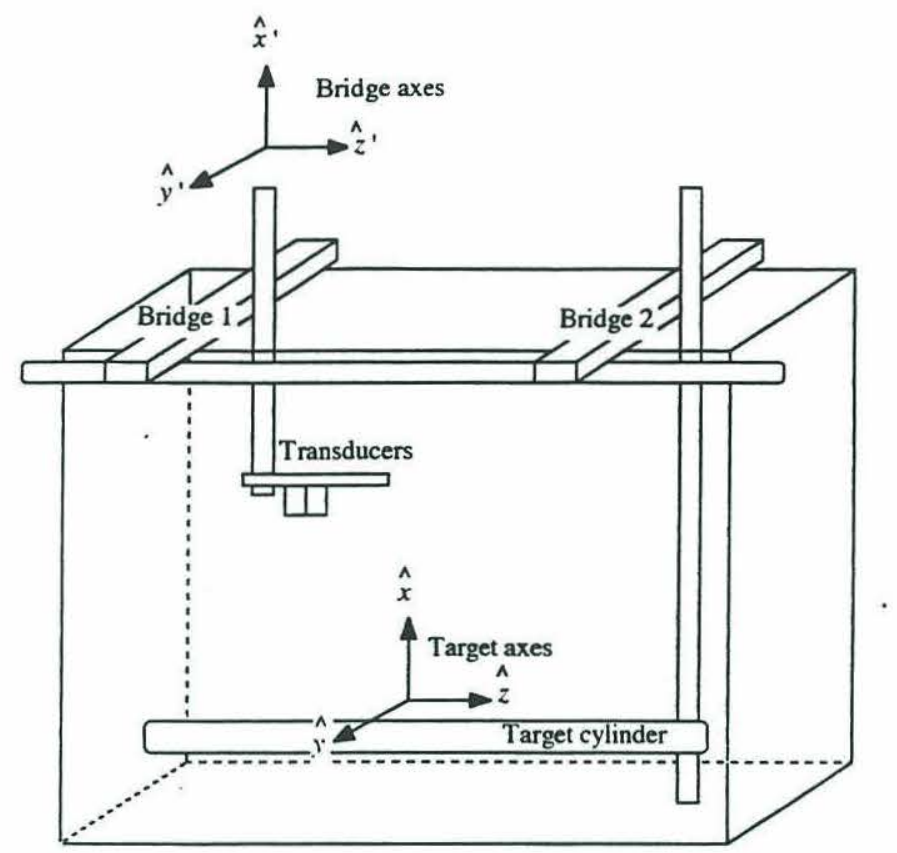

Figure 4-6: Side view of test tank showing mounting of transducers and targets approximating a monostatic backscattering measurement.

tion bridges allowing precise movement of each bridge in any direction. Aligning the target cylinder $z$ axis parallel to the long $z^{\prime}$ axis of the motion bridges (Fig. 4-6), we were able to traverse the transducer mount down the length of the target while holding the orientation constant. In addition, we were able to change the target and mount several different length cylinders and maintain the system alignment. This proved critical considering the very short acoustic wavelengths used for this experiment.

The formation of air bubbles on the transducer faces and target was also a constant problem during the measurements. The tank was filled with fresh water and allowed to sit and degas for a week prior to taking data. This allowed any suspended particulate matter to settle and minimized the amount of gases dissolved in solution. Clay and Medwin [2] derive an approximate relation for air bubble resonance at sea level

$$
f_{\mathrm{R}} \cong \frac{3.25 \times 10^{6}}{a(\mu \mathrm{m})}
$$


where $f_{\mathrm{R}}$ is the resonance frequency and $a$ is the bubble radius. In the frequency band of interest, bubbles between 10.8 and $0.93 \mu \mathrm{m}$ would resonant and corrupt the data. Throughout the experiment, a wetting agent (ordinary liquid hand soap) was applied to the transducer faces and target surface to eliminate any microbubbles on the elements and provide good acoustic coupling to the water.

Two basic geometries were used for these measurements. First a series of beam pattern and calibration measurements were taken with the source and receiver placed facing one another across the tank. The two transducers were mounted on separate bridges and aimed toward one another. The bridges were brought together until the transducer faces touched and final orientation adjustments were made, then one bridge was traversed straight back $30 \mathrm{~cm}$, maintaining the transducer orientation and allowing an accurate measure of the transducer separation. The source was mounted on the end of one of the stepper motor drive shafts so it could be rotated through a large azimuth angle while the separation was held constant (Figures 4-7(a) and 4-8(a)). Measurements were taken through \pm 40 degrees in 2 degree steps.

For the backscattering measurements, the transducers were mounted side by side on one bridge facing down at the target (Figures 4-7(b) and 4-8(b)) mounted $24 \mathrm{~cm}$ below. One target mount was connected to the side wall of the tank and the other mount was connected to the second motion bridge thus allowing the mount separation to be adjusted during the measurements while maintaining the target alignment. During the experiment the backscattered field was measured at several different positions down the length of the target cylinder's axis. Since the transducers had a limited beamwidth, this provided measurements of independent realizations of the random surface profile. Lowering the transducers until they almost touched the target surface and then traversing the transducer bridge down the $z$ axis of the target cylinder, the target was aligned parallel to the $z^{\prime}$ axis of the bridge and oriented normal to the $x^{\prime}$ axis (Fig. 4-6). We were unable to 

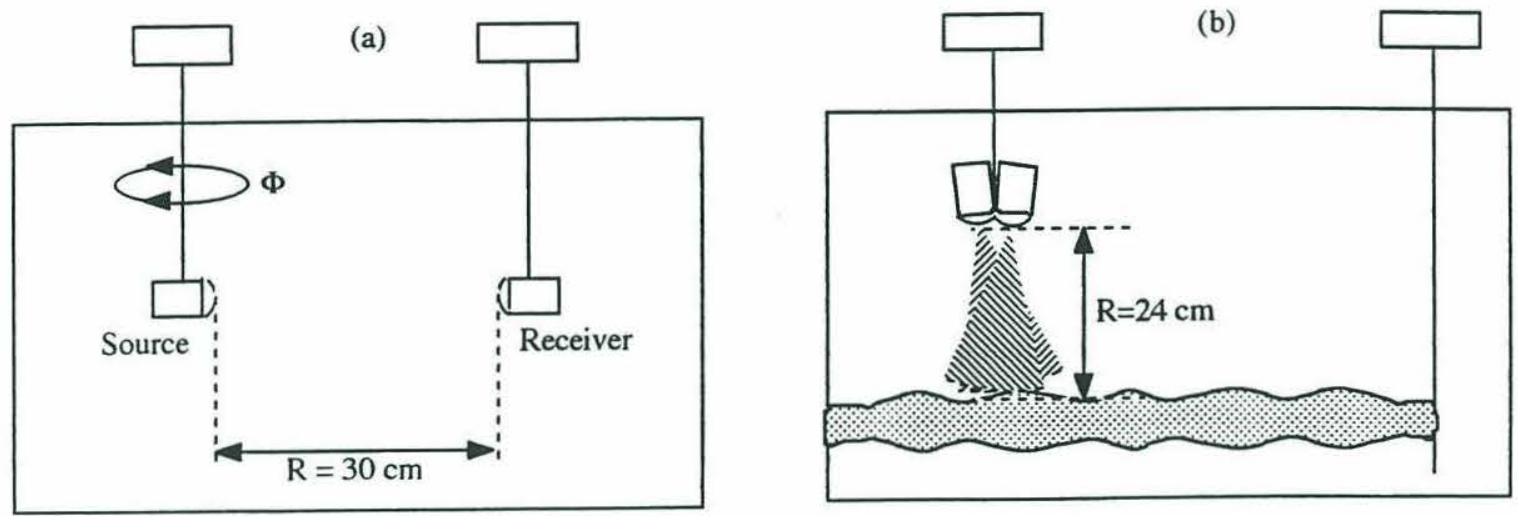

Figure 4-7: Transducer and target configurations for data measurements: (a) for beam pattern measurements, and (b) for rough cylinder backscatter measurements.

accurately measure the transducer aim after mounting them for the rough cylinder measurements, so the beams were mechanical aimed at the target axis and each transducer was angled slightly inward to insure the beams overlapped on the target. Even though the transducer maximum response axes were not exactly normal to the target axis, the relative transducer/target alignment was held constant throughout the experiment.

\subsection{Data acquisition and signal processing}

The data was collected using bandlimited impulse response techniques measuring the frequency response with a single pulse [35]. An impulse was applied to the source transducer exciting it over its entire bandwidth. This transmitted into the water a bandlimited acoustic pulse whose spectrum was shaped by the frequency response of the source transducer. Fourier transforming the received time series yields 


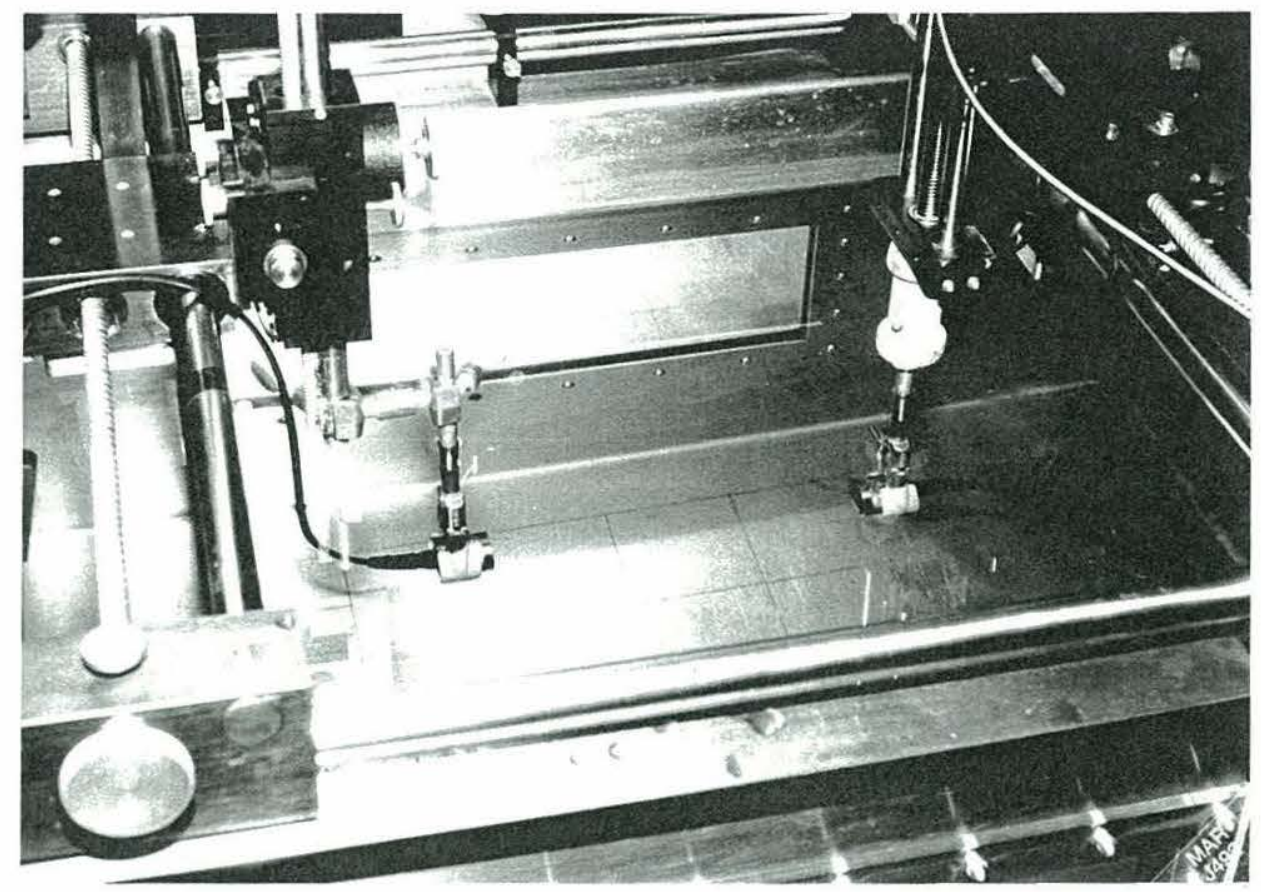

(a) Source beam pattern measurements.

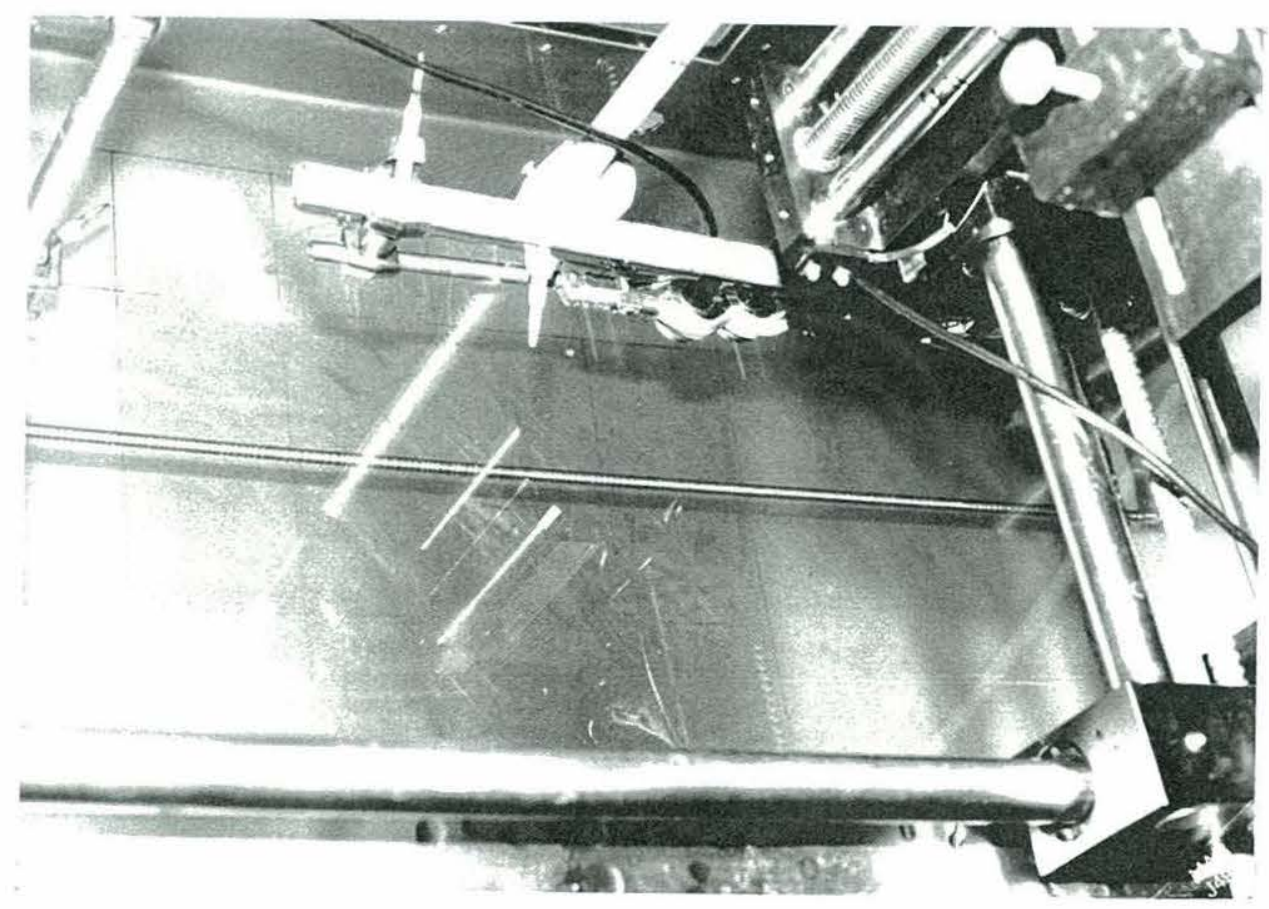

(b) Rough cylinder backscatter measurements.

Figure 4-8: Laboratory setup for experiments. 
the frequency response of the entire transducer-target-electronics system. Deconvolving from this the transducer-electronics impulse response measured during the calibration procedure gives the target frequency response.

The driving impulse was generated by a Parametrics 5055PR pulser connected directly to the source transducer. The pulser contained an internal power amplifier so no additional signal conditioning was required on the transmitter side. The received signals were amplified, filtered and captured with a digital oscilloscope connected to an IBM PC (Fig. 4-9). In order to maximize the signal to noise ratio, the received signal was first amplified through a Parametrics model 5660B preamp providing $+60 \mathrm{~dB}$ of gain. The signal was then high-pass filtered (KrohnHite model $3202 \mathrm{R}$ with cutoff frequency $200 \mathrm{kHz}$ ) to remove all low frequency noise. The final signal was captured and stored on a Analogic Data 6000 digital oscilloscope equipped with a model 630 plug-in module. The oscilloscope sampled the signal at $10 \mathrm{MHz}$ (applying an internal $5 \mathrm{MHz}$ anti-aliasing filter) using 12 bit A/D conversion. An HP 3314 function generator provided an $8 \mathrm{~Hz}$ square wave trigger and timing signal for the entire system and all the equipment was controlled from the computer through a standard GPIB interface. The computer also controlled the bridge motors through an HP 3488 switcher/controller.

The oscilloscope digitized and stored a 1024 point time series of the receiver signal for each ping. At a sampling frequency of $10 \mathrm{MHz}$, this gave a time window of $102.4 \mu \mathrm{sec}$. This time window was delayed (based on the trigger pulse) so that the initial specular reflection arrived near the beginning of the data window allowing as much time as possible to measure later arrivals associated with the Rayleigh modes of the target. The data acquisition sequence consisted of capturing 100 separate pings of the target (at a $8 \mathrm{~Hz}$ pulse repetition rate) with the oscilloscope. These pings were averaged and the averaged time series was stored as a single measurement. The bridges then moved the transducers and/or target into position for the next measurement and the system went into a 5 second wait period to 


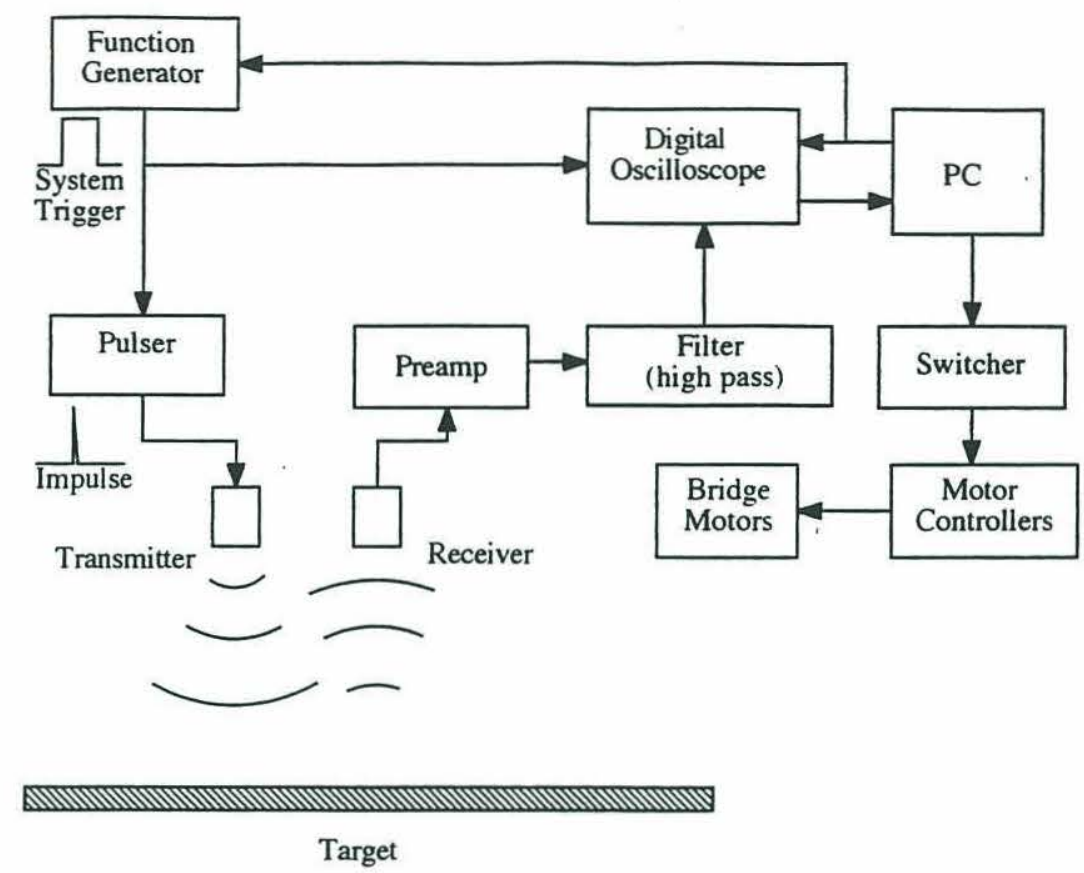

Figure 4-9: Schematic diagram of data acquisition system and motor controllers.

allow any mechanical oscillations in the support frame to decay. Then the entire acquisition cycle repeated. When all the programmed measurements were complete, the bridges returned to their original positions.

Normally when Fourier transforming finite-length time series, one applies a shaped window to the time series to reduce leakage and spectral smearing caused by the data window impulse response [47]. A finite-length time series has an inherent rectangular or boxcar window by virtue of the fact that the data start at some point and stop a finite time later. The leakage and frequency smearing in the spectrum are due to the mainlobe width and sidelobe levels in the window's Fourier transform. The mainlobe width is in turn related to the length of the window and the side lobe levels are related to the taper or shape of the window. Typical data windows such as the Hanning or Bartlett window apply a high degree of amplitude taper over much of the window length to decrease side lobe levels. However when dealing with transient rather than steady-state signals, such a highly tapered window could destroy the entire signal and pass just the noise. We wanted a window that had no 
taper (amplitude $=1$ ) over the time gate when signal arrived but provided some tapering at the beginning and end of the window. We took an 80 point Hanning window, split it.in half, and inserted a 944 point flat (amplitude $=1$ ) section in the middle. This rectangular window with Hanning edges provides some degree of side lobe suppression while giving a long zero taper region for data collection. Figure 4-10 shows the window and its sidelobe roll-off compared to a standard rectangular window.

The recorded measurements were multiplied by this modified Hanning window and the mean value was subtracted from each to remove any bias from the A/D converters. The time series were then zero-padded and transformed using a 2048 point Fast Fourier Transform (FFT) for frequency domain analysis.

\subsection{Calibration of system}

As described in Section 4.2, a series of beam pattern measurements were taken using the 2.25 MHz transducers. The source transducer was mounted to a stepper motor and rotated through $\pm 40^{\circ}$ in $2^{\circ}$ steps while the receiver was held fixed $30 \mathrm{~cm}$ away. Measurements were taken to ensure that no spurious reflections from the side walls or the water surface were received during the data time window. For the beam pattern measurements, the signal to noise ratio was very high and only 64 pings were averaged rather than the 100 pings used for backscatter measurements as described in Section 4.3. Figure 4-11 shows the time series measured over the full azimuth range where all time measurements are referenced to the source trigger pulse. The source transducer moved in a small arc since its center axis was not exactly aligned with the drive shaft. This caused the $4 \mu \mathrm{sec}$ deviation in the arrival of the leading edge of the pulse evident from about $\Phi=-6^{\circ}$ to $30^{\circ}$. The measured sound speed in the water was $1466 \mathrm{~m} / \mathrm{sec}$ so this $4 \mu \mathrm{sec}$ deviation means the transducer face moved $5.86 \mathrm{~mm}$ during the measurement or a relative error of $2 \%$. The faint late arrivals 

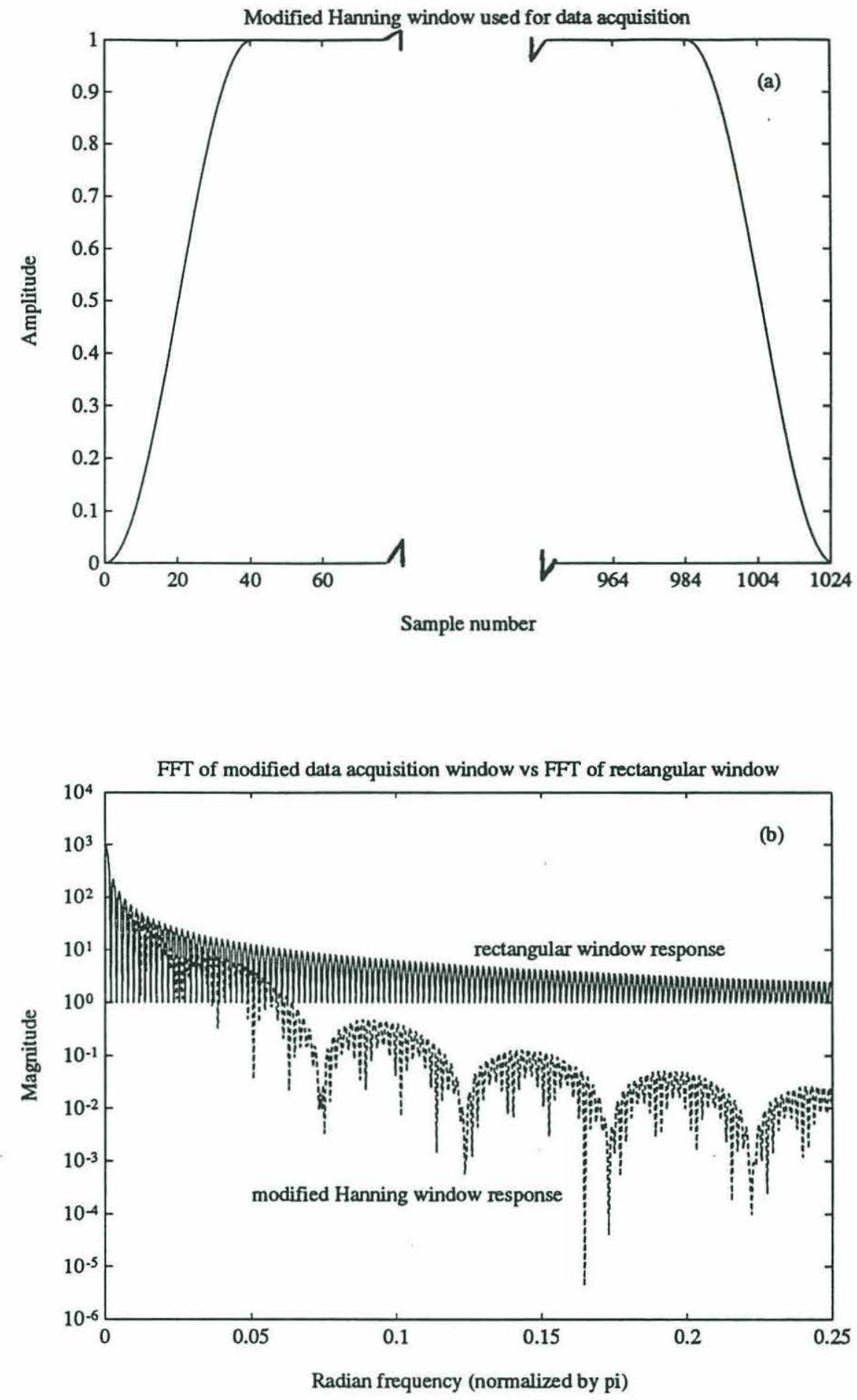

Figure 4-10: Modified Hanning window used in data acquisition: (a) close-up of leading edge (and trailing edge) of 1024 point window for time series showing smoothing due to the applied 40 point Hanning edge.(b) Fourier transform of modified Hanning window vs rectangular window. 


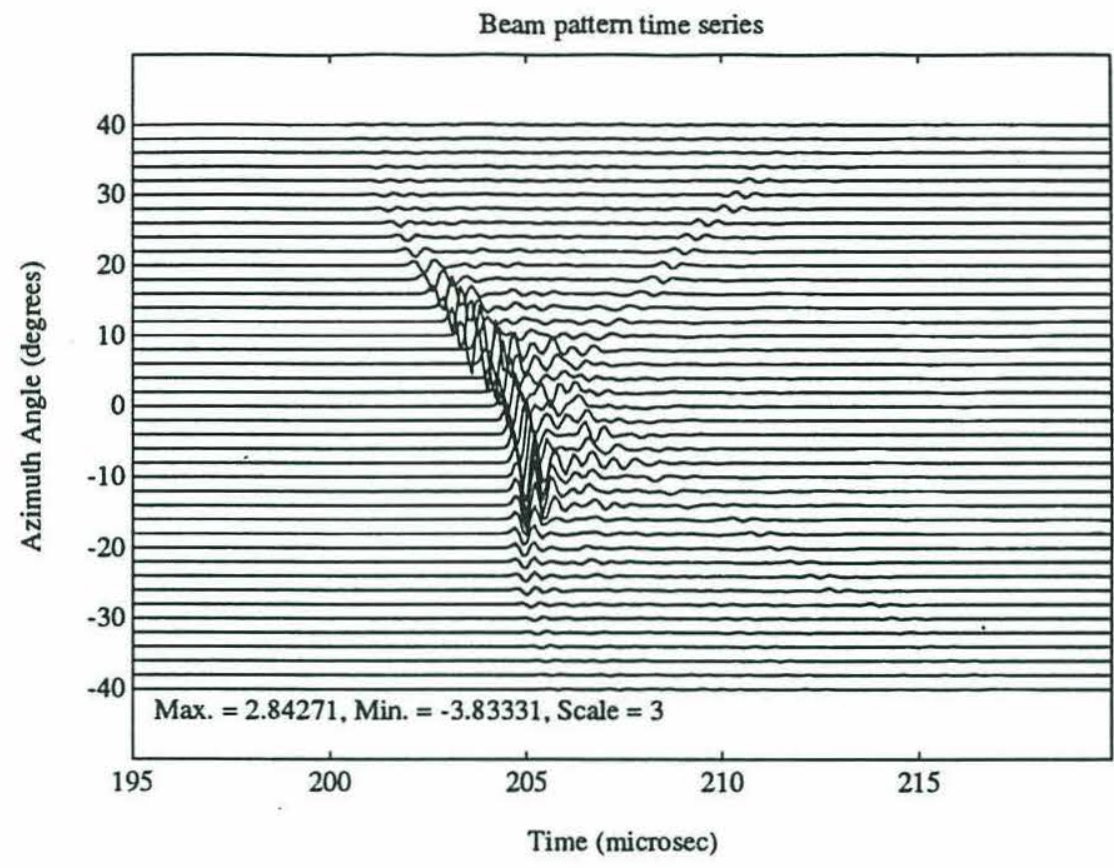

Figure 4-11: Waterfall plot of time series from beam pattern measurement.

seen trailing out to $215 \mu \mathrm{sec}$ are due to radiation from the back of the transducer. Ambient noise measurements were also taken with the same equipment setup and the source turned off.

Fourier transforming these data give the source frequency response as a function of both frequency and azimuth. Since we did not have a calibrated reference hydrophone available, the maximum value in the data was set to $0 \mathrm{~dB}$ and used as the normalization constant. In Fig. 4-12, we can see that the response extends from about $3.5 \mathrm{MHz}$ down to the high-pass filter roll-off at $250 \mathrm{kHz}$. The beam pattern extends out to about $\Phi= \pm 20^{\circ}$ with the maximum response at about $8^{\circ}$ off the beam axis. These data have not been normalized to a reference distance; that will be accounted for in the later analysis. Using the measured ambient noise time series, Fig. 4-13 shows the signal to noise ratio for these measurements was well above $20 \mathrm{~dB}$ for almost all frequencies within $\pm 20^{\circ}$ of azimuth.

The greyscale plots show the high degree of fluctuation in the source response near its center axis. Figure 4-14 shows the frequency response above $1.25 \mathrm{MHz}$ 


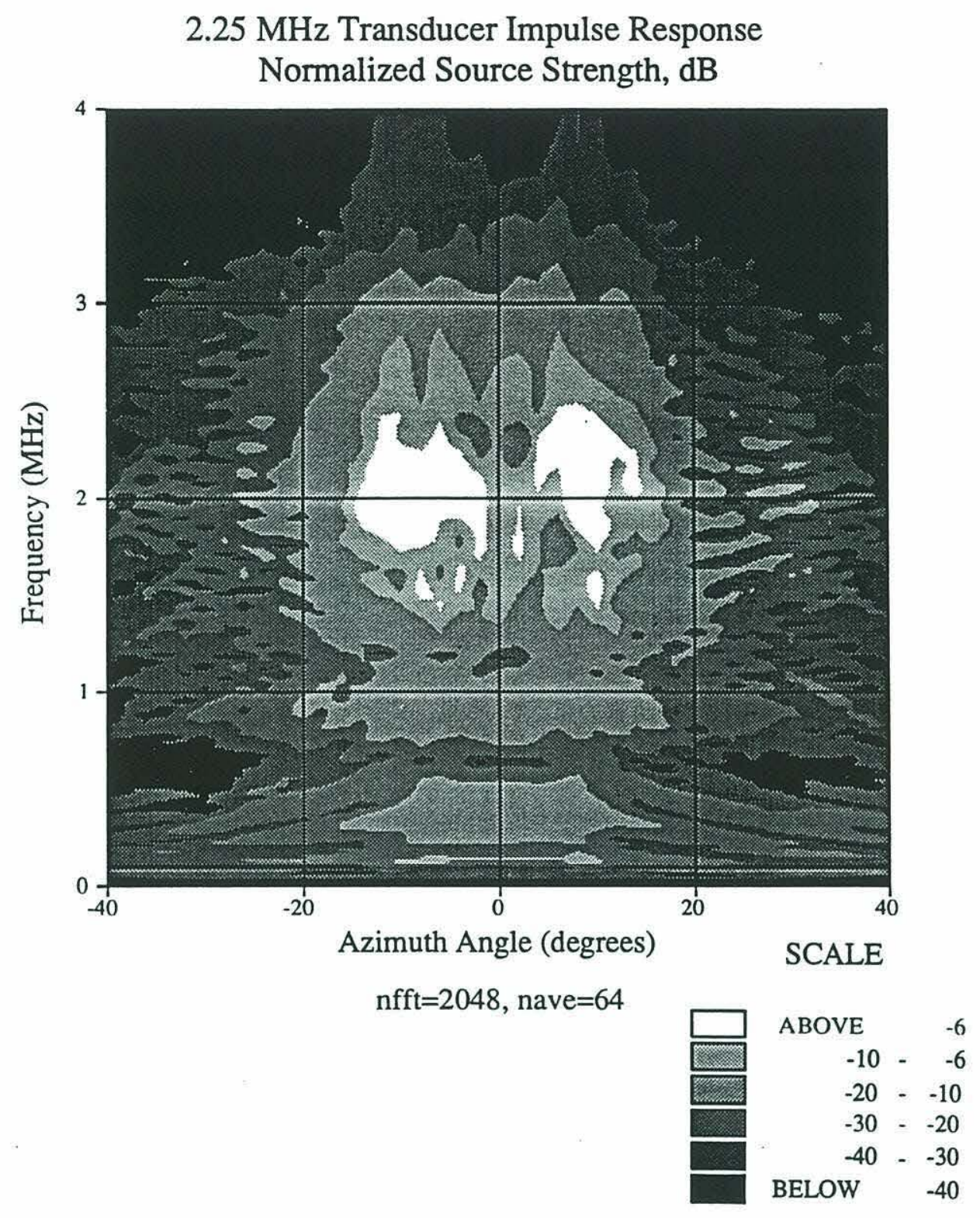

Figure 4-12: Greyscale plot of Fourier transformed beam pattern time series showing frequency response vs. frequency and azimuth angle $(\Phi)$ for transducer from $-40^{\circ} \leq \Phi \leq 40^{\circ}\left(\Phi=0^{\circ}\right.$ is "broadside"). 


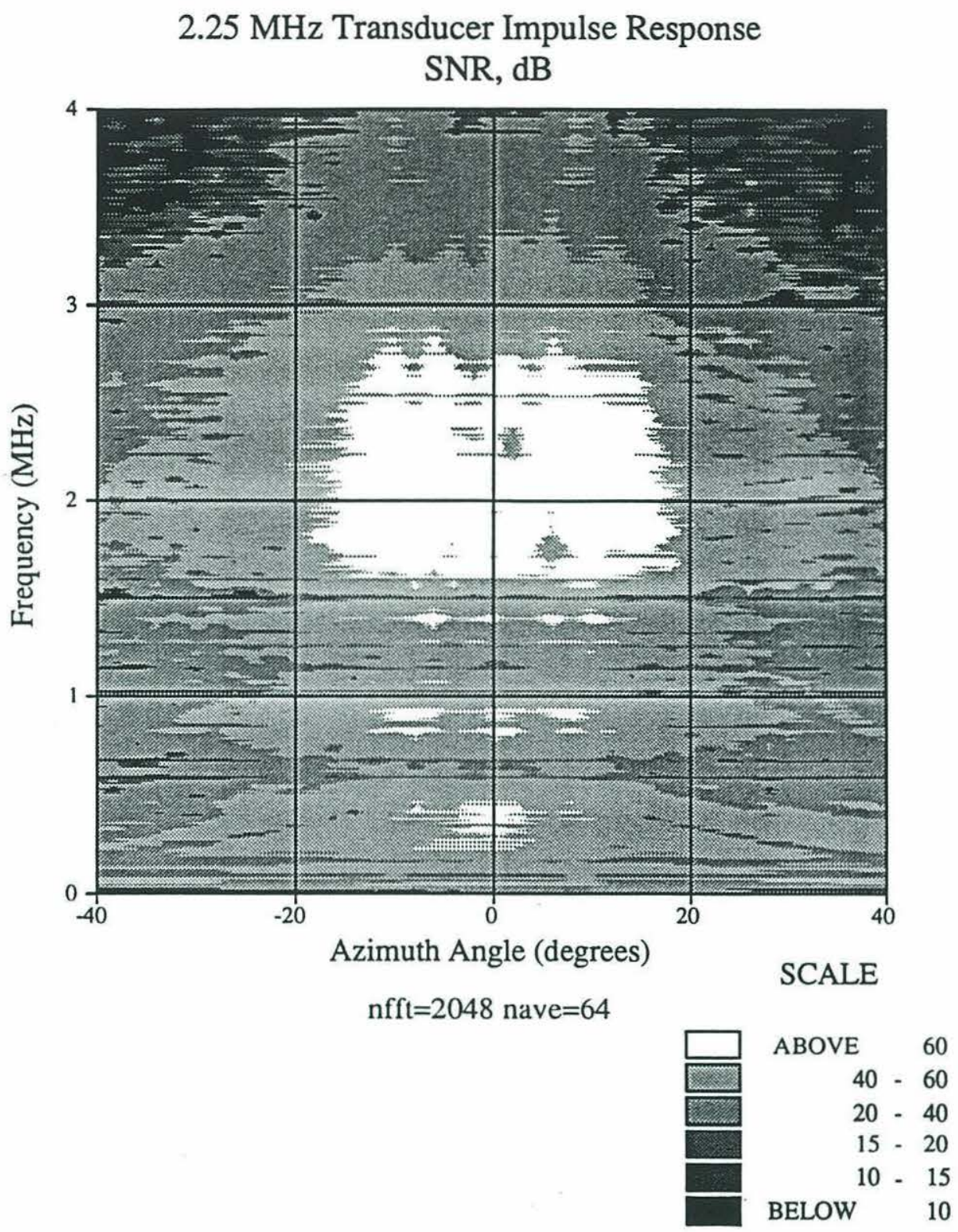

Figure 4-13: Greyscale plot of signal to noise ratio (SNR) for beam pattern measurements vs frequency and $\Phi$. 


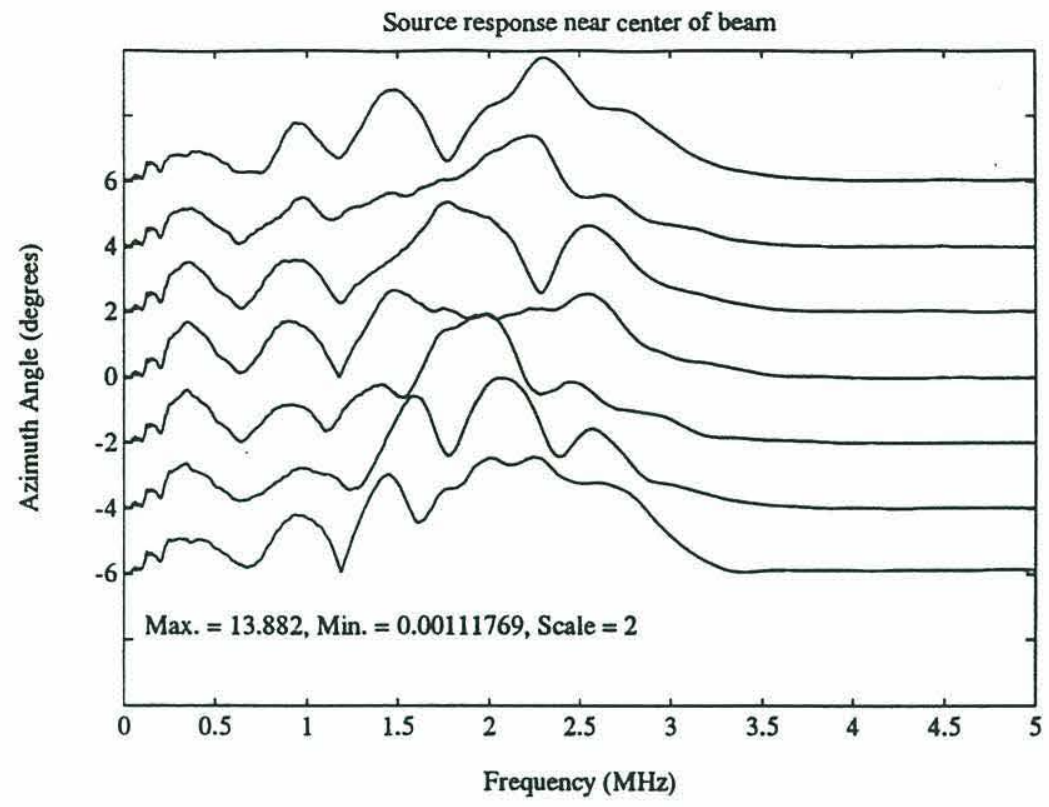

Figure 4-14: Frequency response measurements for near center beam $\left(\Phi \leq \pm 6^{\circ}\right)$.

is highly variable even over two degrees and is not symmetric about $\Phi=0^{\circ}$. We believe the center beam null is due to internal reflections in the transducer element which give rise to destructive interference right on the beam axis. The time series show that this internal reflected arrival is confined to $\pm 4^{\circ}$ in the center of the beam. Since the target spans the entire transducer beam (as required to consider the target as infinitely long), this irregular source pattern greatly complicates the calibration process. For any given measurement of the infinitely long cylinder, the incident field at each point on the surface is a complicated function of both frequency AND angle as shown in Fig. 4-12. Averaging the source response over the main frequency band (Fig. 4-15) shows a Gaussian model approximates the roll off in the "average" beam pattern but does reflect the bimodal structure in the center beam. This question of system calibration was partially answered by using backscatter data from the smooth cylinder as a reference standard. 


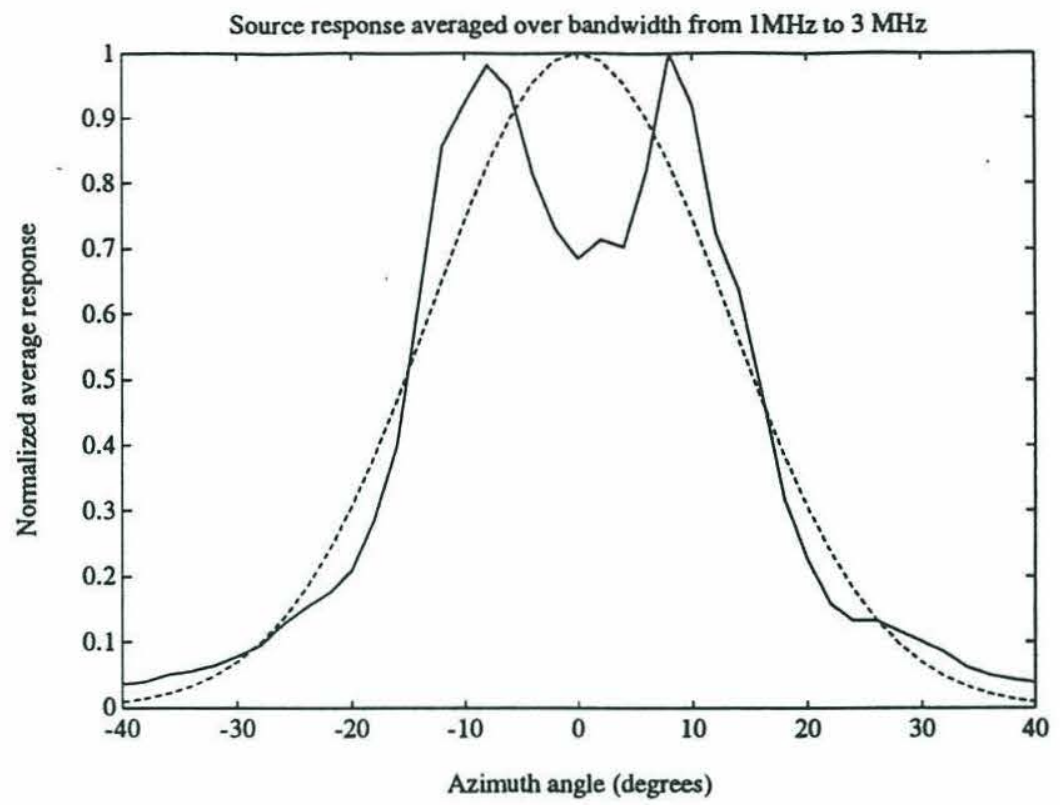

Figure 4-15: Transducer frequency response averaged over frequency to give "average" beam pattern vs $\Phi$. The response was averaged over the bandwidth from 1 $\mathrm{MHz}$ to $3 \mathrm{MHz}$. A fitted Gaussian (- - ) with characteristic beamwidth $\Theta_{o}=26^{\circ}$ is superimposed for comparison.

\subsection{Experimental data and data selection}

As noted earlier, the scattering data was collected with a transducer to target range of $24 \mathrm{~cm}$, and the measured sound speed in the tank was $1477 \mathrm{~m} / \mathrm{sec}$ for the duration of the scattering experiments. This gives a first Fresnel zone size of $5.4 \mathrm{~cm}$ at 250 $\mathrm{kHz}$ down to $1.4 \mathrm{~cm}$ at $3.5 \mathrm{MHz}$. Using the $26^{\circ}$ beamwidth estimated in Fig. 415, the beam is $11.1 \mathrm{~cm}$ wide at the target. Thus, the criteria for considering the cylinder as effectively infinite discussed in Section 2.2 are met: the target $(40 \mathrm{~cm}$ smooth cylinder and $75 \mathrm{~cm}$ rough cylinder) is long enough to completely span the beamwidth and the beamwidth is wide enough to span several Fresnel zones.

Three separate data runs were made measuring backscattering from the rough cylinder target. A different target configuration was used for each run to maximize the number of independent realizations (Fig. 4-16). Although there is some overlap of target segments between data runs, we feel these still provide independent real- 


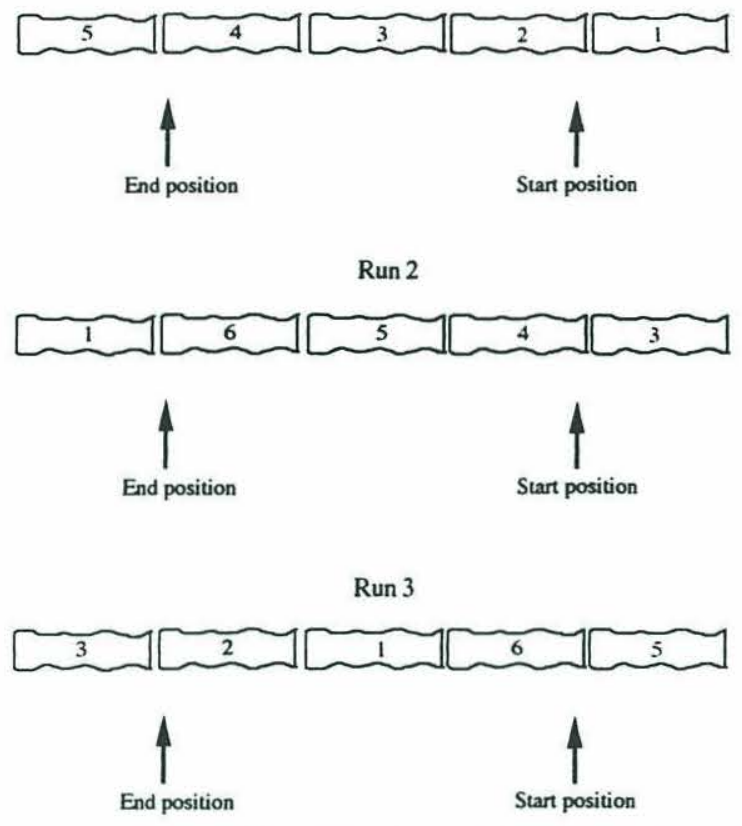

Figure 4-16: Configuration of rough cylinder subsections for data runs 1 through 3.

izations since the target correlation length is so small (Fig. 4-5). Since the rough cylinder target had to be removed from the mounts to rearrange the subsections, alignment and calibration measurements were made prior to each rough surface data run to ensure the orientation and alignment had not changed. The $40 \mathrm{~cm}$ smooth cylinder was mounted and used as a standard for these alignment measurements. Each of the alignment checks consisted of making a backscatter measurement (average of 100 pings) at 4 positions along the axis of the smooth cylinder target. The first measurement position was directly over the joint between the subsections and the remaining measurements were taken at $3 \mathrm{~cm}$ intervals down the $z$ axis of the target. By comparing the arrival times between the first and last position, we ensured that the target axis $z$ remained parallel to the axis of bridge motion $z^{\prime}$. In addition, comparing the arrival times between separate alignment runs, we were able to verify that the overall alignment was unchanged even though the target was removed and remounted in a different configuration.

Figure 4-17 shows a waterfall plot of the time series from all four of the the 


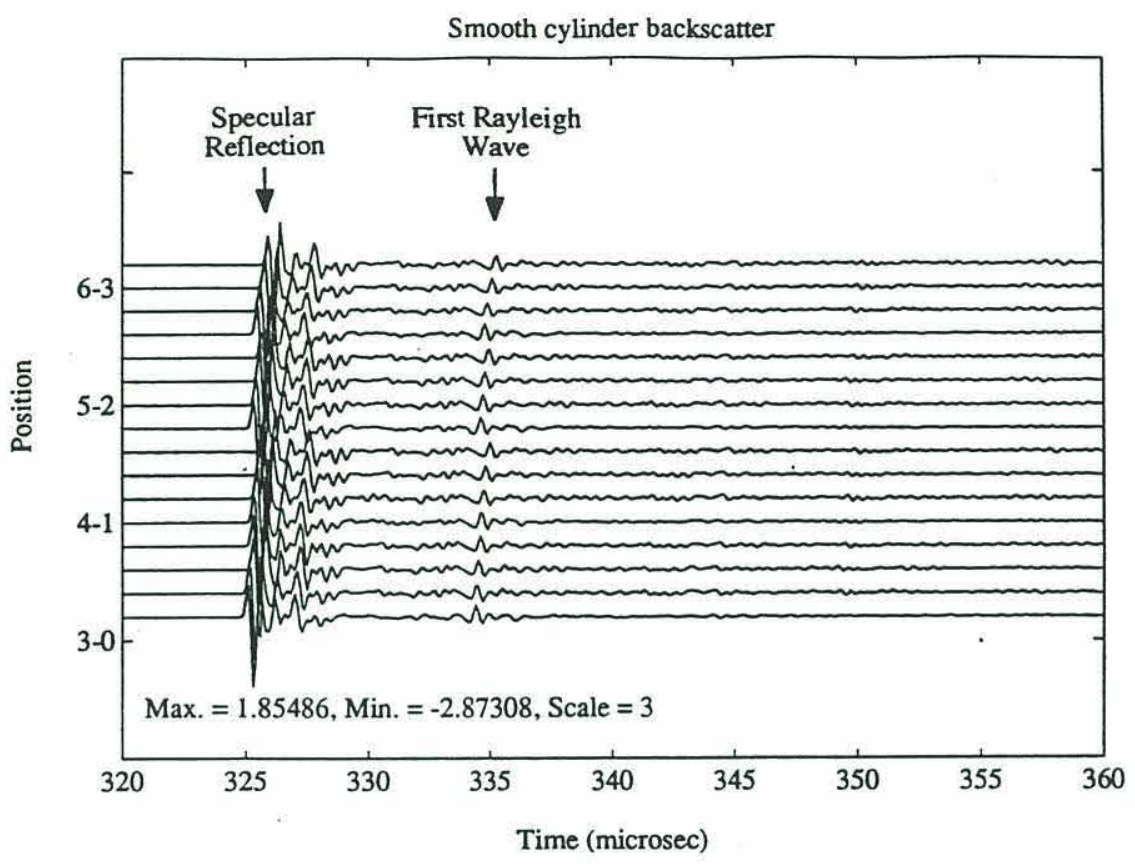

Figure 4-17: Time series for smooth cylinder alignment and calibration measurements taken throughout the experimental run. Position 1 was directly over the joint between the two $20 \mathrm{~cm}$ smooth cylinder subsections. The transducers were moved $2 \mathrm{~cm}$ along the $z^{\prime}$ axis toward the end of the target between positions.

smooth cylinder alignment checks. Each measurement is labeled where the first number represents the calibration run number and the second is the transducer position within that run. This figure illustrates the fact that the precise alignments were maintained; the initial arrival deviates less than $0.8 \mu \mathrm{sec}$ over the whole experiment. Figure 4-17 also shows the separate arrivals of the different scattering modes discussed in Section 2.1.3. The first Rayleigh surface elastic wave is clearly evident and labeled on the figure. Higher order Rayleigh waves corresponding multiple circumnavigations of the cylinder and possibly Whispering Gallery waves can be seen at $t=344,350$, and $357 \mu$ sec.

Using the phase shifts derived in Section 2.1.3 (Eqs. 2.52, 2.55, and 2.56), we can calculate the expected delay of the Rayleigh wave arrival relative to the specular 
reflection arrival. The phase shift for the specular component was

$$
\eta_{\text {specular }}=-2 k a
$$

and for the $m$ th Rayleigh wave

$$
\eta_{R}=k a\left[\left(c / c_{R}\right)\left(2 \pi-2 \theta_{R}\right)-2 \cos \theta_{R}\right]+2 \pi m k a c / c_{R}
$$

The phase difference between these two waves is

$$
\Delta \eta=2 k a\left\{\left(\cos \theta_{R}-1\right)-\left(c / c_{R}\right)\left(\pi(m+1)-\theta_{R}\right)\right\}
$$

This corresponds to a time delay

$$
\Delta t=(2 a / c)\left\{\left(\cos \theta_{R}-1\right)-\left(c / c_{R}\right)\left(\pi(m+1)-\theta_{R}\right)\right\} .
$$

Neubauer [35] gives $c_{R}=2827 \mathrm{~m} / \mathrm{sec}$ for stainless steel. This gives a Rayleigh "launch" angle of $\theta_{R}=31.5^{\circ}$. Calculating the expected delay for the first three Rayleigh waves, we find

$$
\begin{aligned}
\Delta t_{m=0} & =9.28 \mu \mathrm{sec} \\
\Delta t_{m=1} & =19.42 \mu \mathrm{sec} \\
\Delta t_{m=2} & =29.56 \mu \mathrm{sec}
\end{aligned}
$$

and these agree with the relative arrivals seen in Fig. 4-17.

Tank echo measurements were taken to ensure that no reflections from the water surface, tank walls, or support structures arrived within the data time window. Figure 4-18 shows the time series recorded at the 4 transducer positions used for the alignment measurements. The same setup and programmed motions were used only the target cylinder was removed from the mounts. There is some reflection off of the target mounts in position 4 and this corresponds to the transducers positioned very close to the end bracket but the signal is more than two orders of magnitude less than the target signal. 


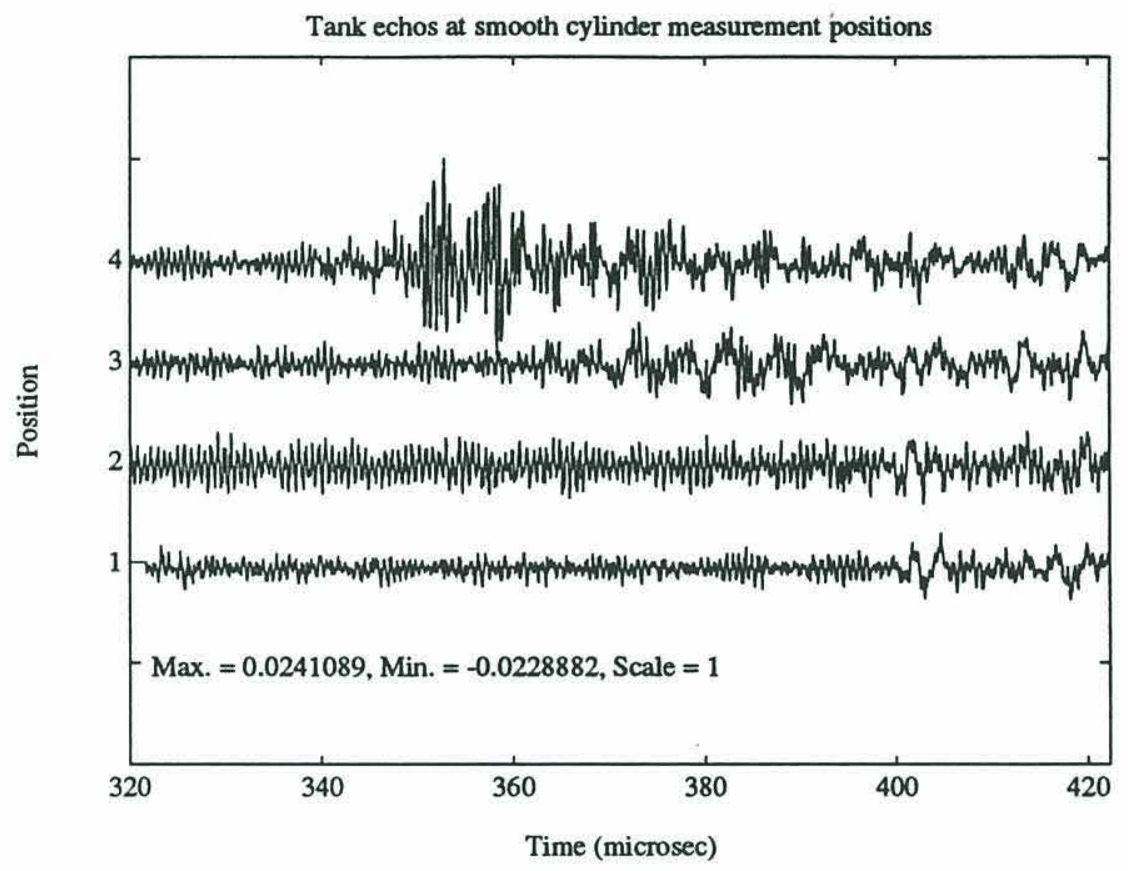

Figure 4-18: Tank echo measurements using exactly the same alignment and transducer motions as Figure 4-17 except with the target removed from the mounts. This shows some return off of the mounts at the extremum position 4 . The returns are roughly $40 \mathrm{~dB}$ below that from the cylinder. 


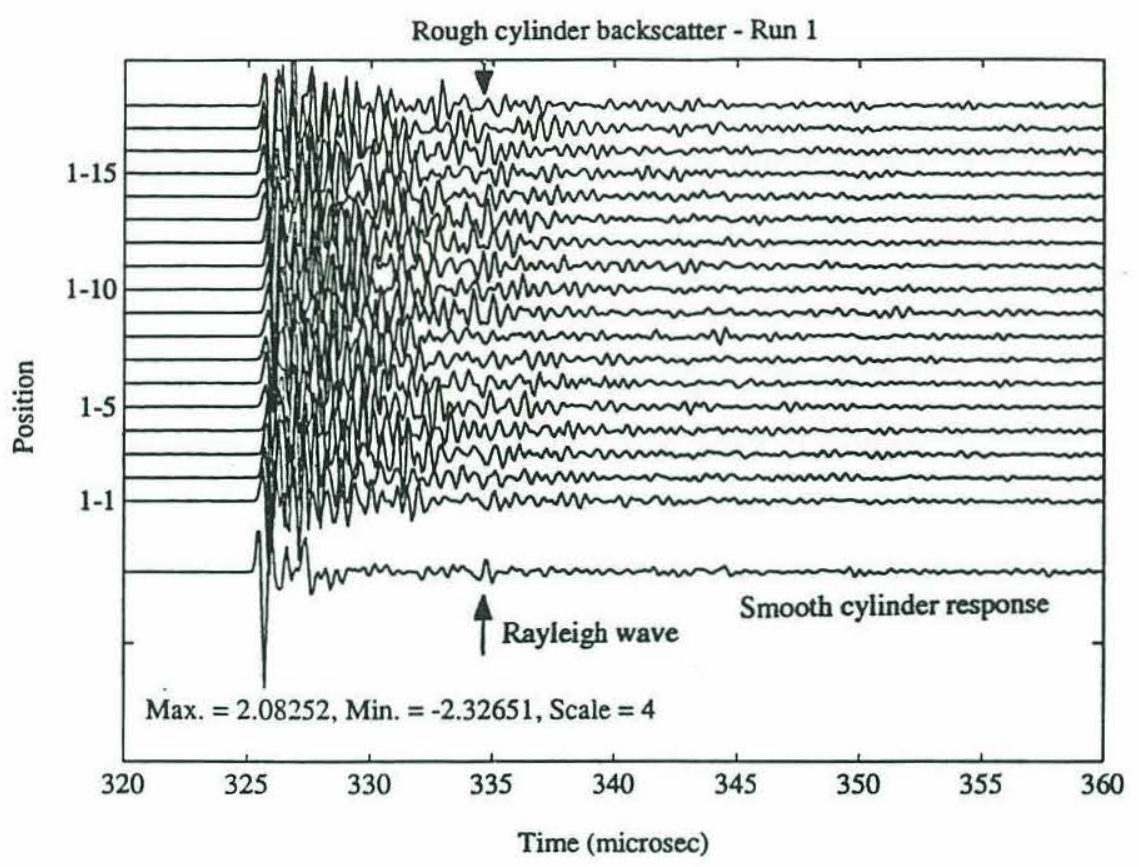

Figure 4-19: Time series for rough cylinder measurements in run 1. The transducer mount was moved $2 \mathrm{~cm}$ along the $z^{\prime}$ axis between measurements and the target configuration is shown in Figure 4-16. A typical smooth cylinder time series is shown to compare the Rayleigh wave arrival times.

Rough cylinder measurements were made at 18 positions spaced $2 \mathrm{~cm}$ apart down the target axis. Figure 4-16 shows the target configuration for each of the three data runs and the approximate starting and ending positions of transducers. Tank echo measurements made at each of the 18 positions showed no reflections from the tank or mounting frame were received during the data window even at the extreme positions 1 and 18. Figures 4-19-4-21 show waterfall plots of the rough cylinder backscatter time series. An typical smooth cylinder time series is included indicating the expected arrival time of the first Rayleigh surface elastic wave.

Again ambient noise measurements were taken through out the experiment by turning off the source and measuring the noise field. Averaging these, an average noise level was found and SNR levels were computed for each measurement. Sample SNR levels are shown in Fig. 4-22 for the smooth cylinder and rough cylinder measurements. The SNR remains well above $+20 \mathrm{~dB}$ from the filter roll-off at 250 


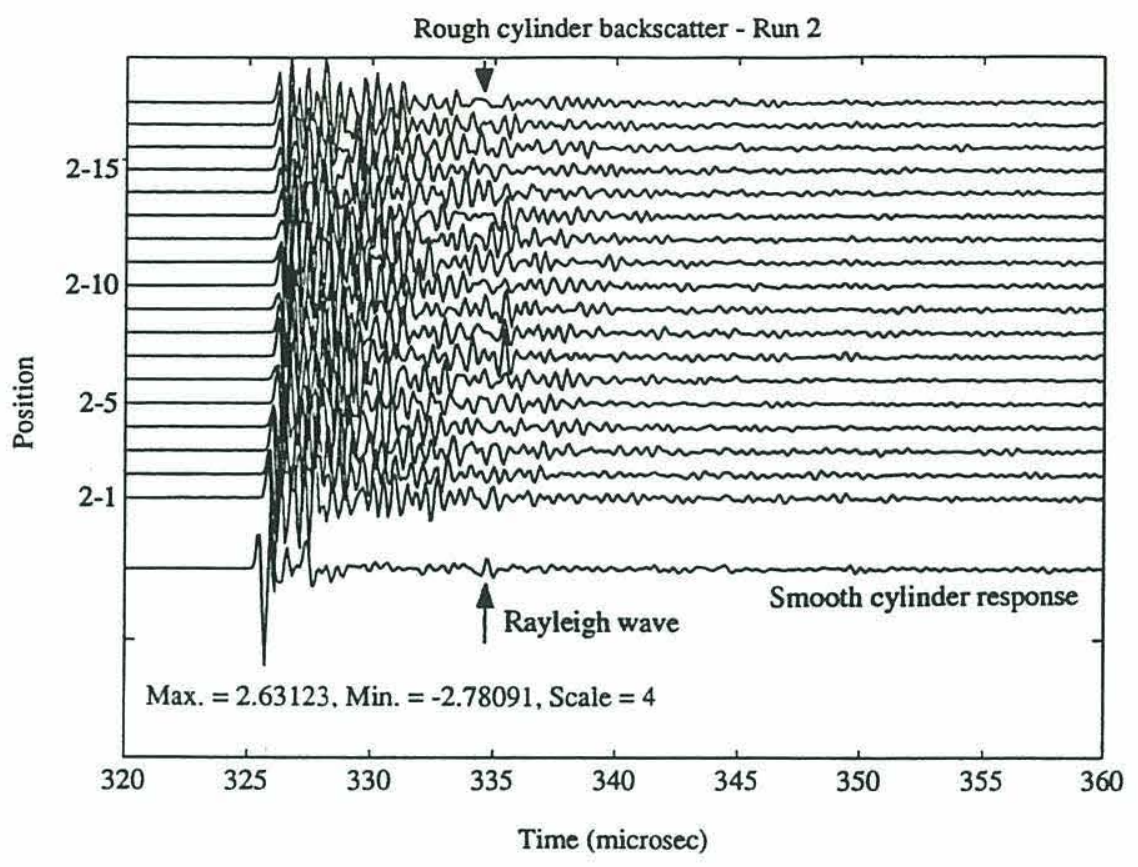

Figure 4-20: Time series for rough cylinder measurements in run 2. A typical smooth cylinder time series is shown to compare the Rayleigh wave arrival times.

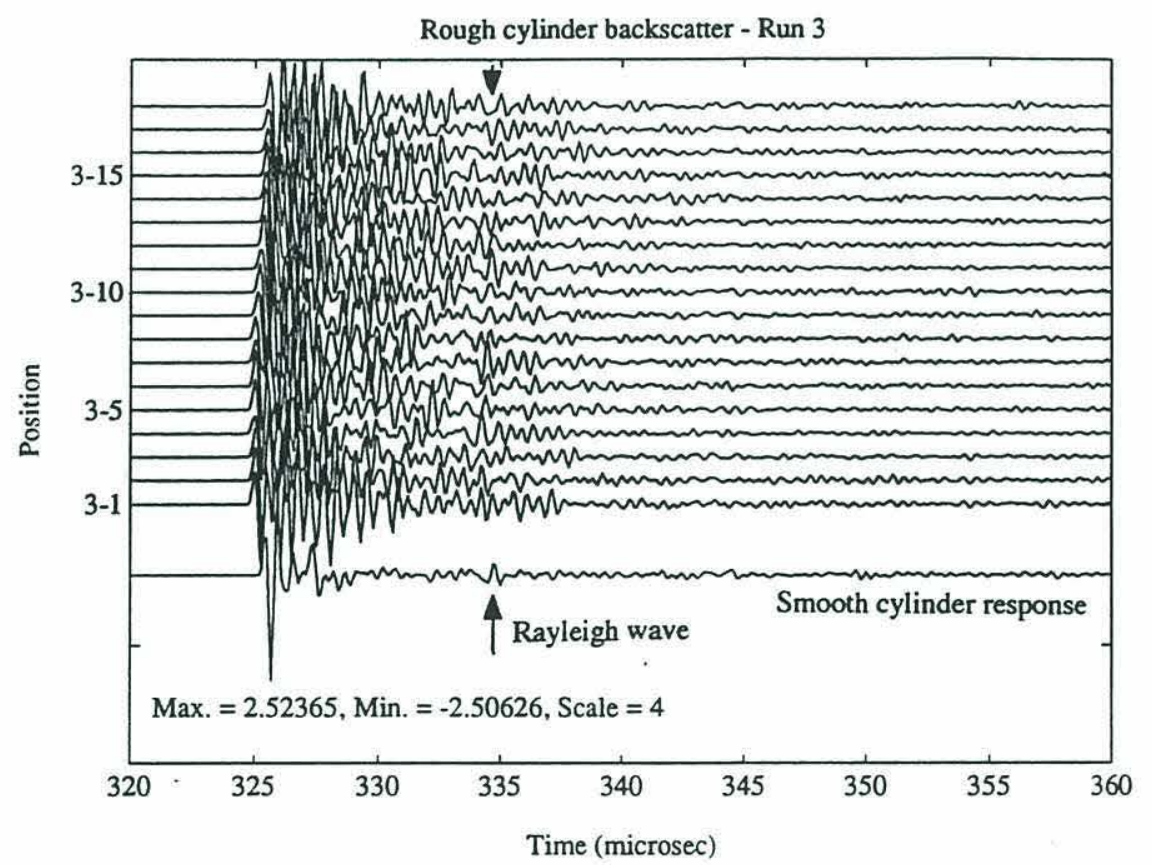

Figure 4-21: Time series for rough cylinder measurements in run 3. A smooth cylinder time series is shown to compare the Rayleigh wave arrival times. 
$\mathrm{kHz}$ to $3.4 \mathrm{MHz}$ except for some low SNR regions between $600 \mathrm{kHz}$ and $1.5 \mathrm{MHz}$.

Fourier transforming the time series gives the frequency response in the backscattered field. However the issue of calibrating this response to normalize for the frequency response of the transducers themselves has not been resolved. The analysis in Section 4.4 showed the response curves for the transducers were highly variable over frequency and angle; efforts to use this data to find some sort of spatially averaged "average effective" incident field were not successful. Another normalization technique that has been used is to calibrate the system based on the measured response from a known "standard" target, typically solid tungsten carbide spheres [35]. The measured response from the standard is compared with an analytic solution to give a normalization or calibration curve for the system and transducers. Similarly, the response from a smooth elastic cylinder is well known so measurements from the smooth cylinder alignment checks were used to find a calibration curve reflecting an " average effective" incident field over the insonified length of the target.

As shown in Eq. (2.80), the scattered field can be represented as a non-dimensional form function $f^{\infty}$

$$
f^{\infty}=\sqrt{\frac{2 R}{a_{o}}} \frac{P_{\mathrm{scat}} R}{P_{o} R_{o}} e^{-i 2 k R}
$$

where $R$ is the distance from the target to the source/receiver and $P_{o}$ is some reference calibration response measured at a distance $R_{o}$. Equation (4.7) assumes a point source with a spherically spreading incident field and a cylindrically spreading reflected field. For short pulse transient measurements the reflected field is given by $g_{r}$ which is the Fourier transform of the reflected pulse and the incident field is $g_{o}$, the transform of the incident pulse at a reference distance $R_{o}$. The form function is then given by

$$
\left|f^{\infty}\right|=\sqrt{\frac{2 R}{a_{o}}} \frac{\left|g_{r}\right| R}{\left|g_{o}\right| R_{o}} .
$$

Since the analytic solution for a smooth elastic cylinder, $f_{c a l}^{\infty}$, is well known, $g_{0}$ can be found from the calibration measurement $g_{c a l}$ of the backscatter from the smooth 

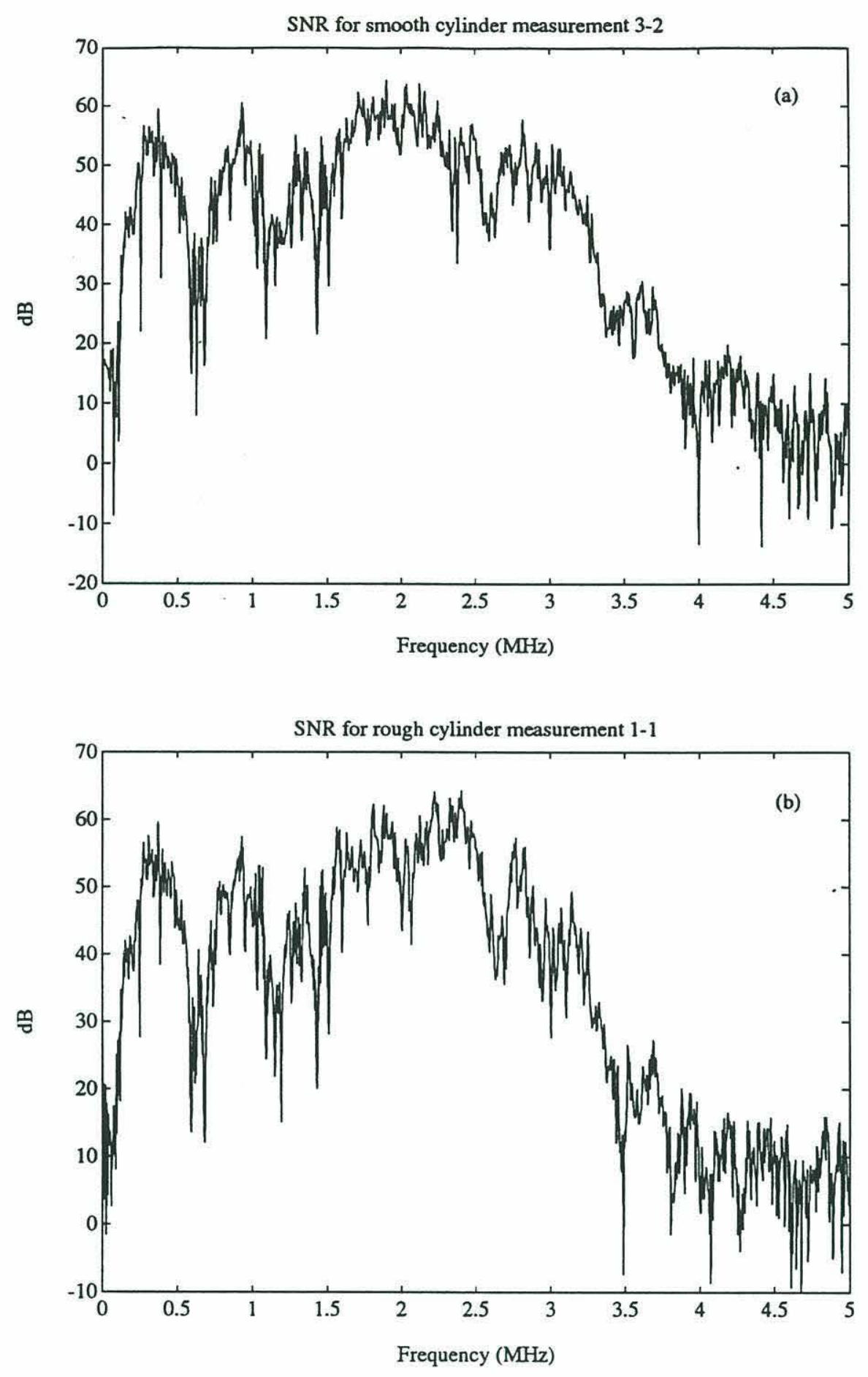

Figure 4-22: Sample SNR levels for (a) smooth cylinder and (b) rough cylinder measurements. 
cylinder

$$
\left|g_{o}\right|=\sqrt{\frac{2 R}{a_{o}}} \frac{\left|g_{c a l}\right| R}{\left|f_{c a l}^{\infty}\right| R_{o}} .
$$

In this case $R_{o}=R=24 \mathrm{~cm}$ so Eq. (4.8) becomes

$$
\left|f^{\infty}\right|=\frac{\left|g_{r}\right|}{\left|g_{\text {cal }}\right| /\left|f_{c a l}^{\infty}\right|} .
$$

Using the material properties for stainless steel given in Table $4.2\left|f_{\text {cal }}^{\infty}\right|$ was calculated and compared to the measured response from the smooth cylinder data $\left|g_{\text {cal }}\right|$. Selecting smooth cylinder measurement 3-2 made away from the joint and the ends, a calibration response curve $\left|g_{c a l}\right| /\left|f_{c a l}^{\infty}\right|$ was calculated (Fig. 4-23a). This figure shows a broad "noisy" response curve where the fine structure or "noise" is due to slight mismatches in the nulls between $g_{c a l}$ and $f_{c a l}^{\infty}$. Low pass filtering this curve (Fig. 4-23b) smoothes the data, removes all the fine structure, and reveals a slowly varying calibration curve corresponding to some "spatially averaged" incident field. Comparison of the calibration curve to actual beam pattern measurements in Fig. 4-14 are shown on Fig. 4-24. Here the beam pattern measurements have been multiplied by a gain factor to account for the fact that the measurements were made at different ranges using different source levels. However, the gain factor

$$
G=\frac{A_{\text {beam }} r_{\text {beam }}}{A_{\text {targ }} r_{\text {targ }}} \sqrt{\frac{a_{0}}{2 r_{\text {targ }}}}=1.755
$$

was determined directly from the equipment set-up data not by empirically fitting the amplitudes of the two curves.

The close match in both structure and amplitude between the curves in Fig. 4-24 indicate that the calibration curve (Fig. 4-23b) is a valid representation of the incident field at least up to $1.5 \mathrm{MHz}$. The mismatch above $1.5 \mathrm{MHz}$ is again due to the highly variable nature of the beam pattern. We were unable to find an acceptable method for calibrating the data above $1.5 \mathrm{MHz}$ to any absolute scale. The relative variations in the scattered fields will be shown for the entire frequency bandwidth however comparisons to analytic solutions will only be valid over the extent of the validity of the calibration curve (up to $1.5 \mathrm{MHz}$ or $k a=30$ ). 

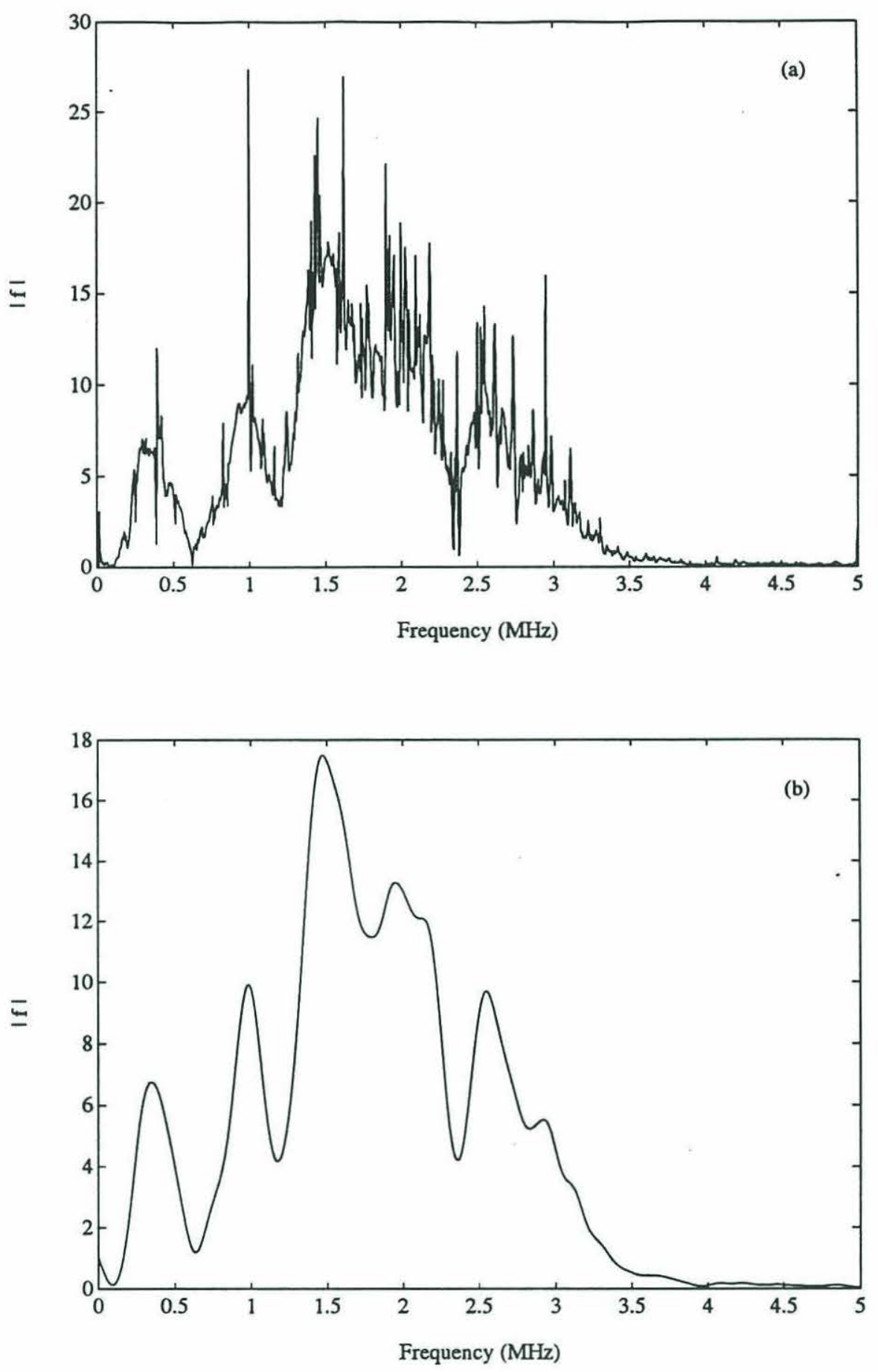

Figure 4-23: Calibration curves obtained by inverting the smooth cylinder "standard" frequency response from Figure 4-17 (measurement 3-2) and the numerical solution for elastic cylinder scattering . (a) Raw curve showing fine structure due to mismatch between nulls. (b) Curve after low-pass filtering using a fourth order Butterworth filter to smooth the data. 

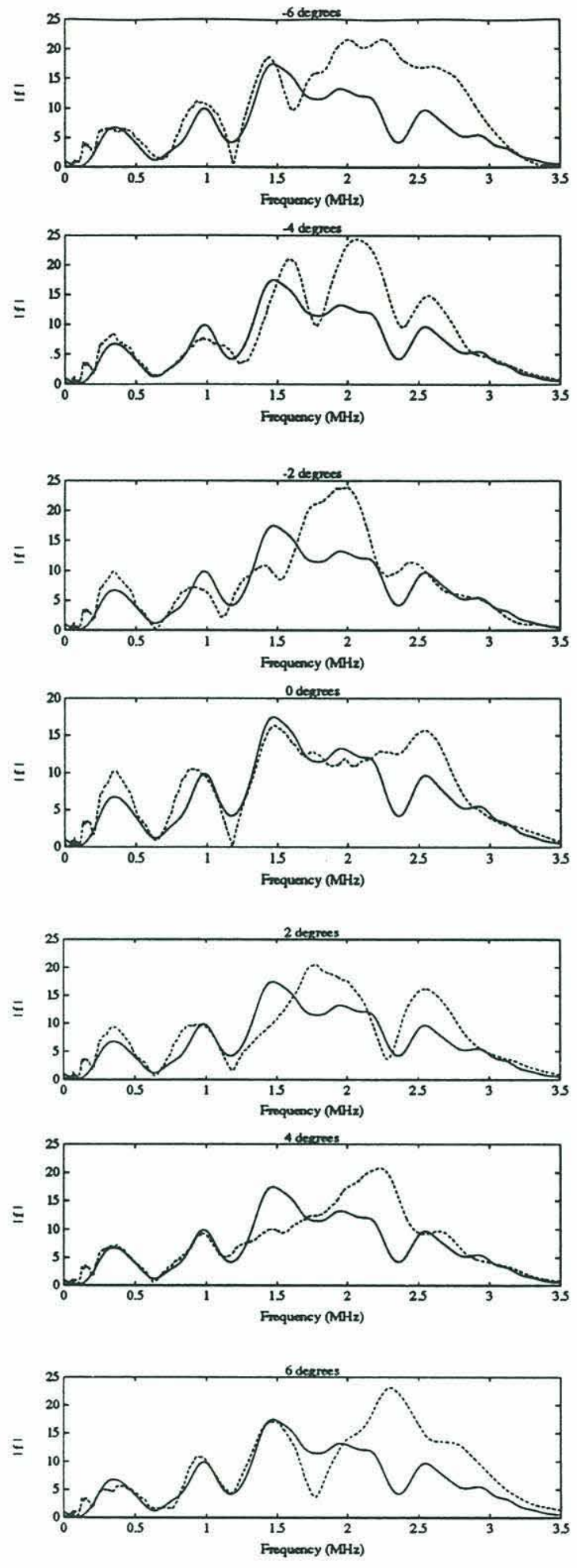

Figure 4-24: Comparison of inverted calibration curve $(-)$ and measured response from beam pattern measurements at different azimuth angles(- - -). 


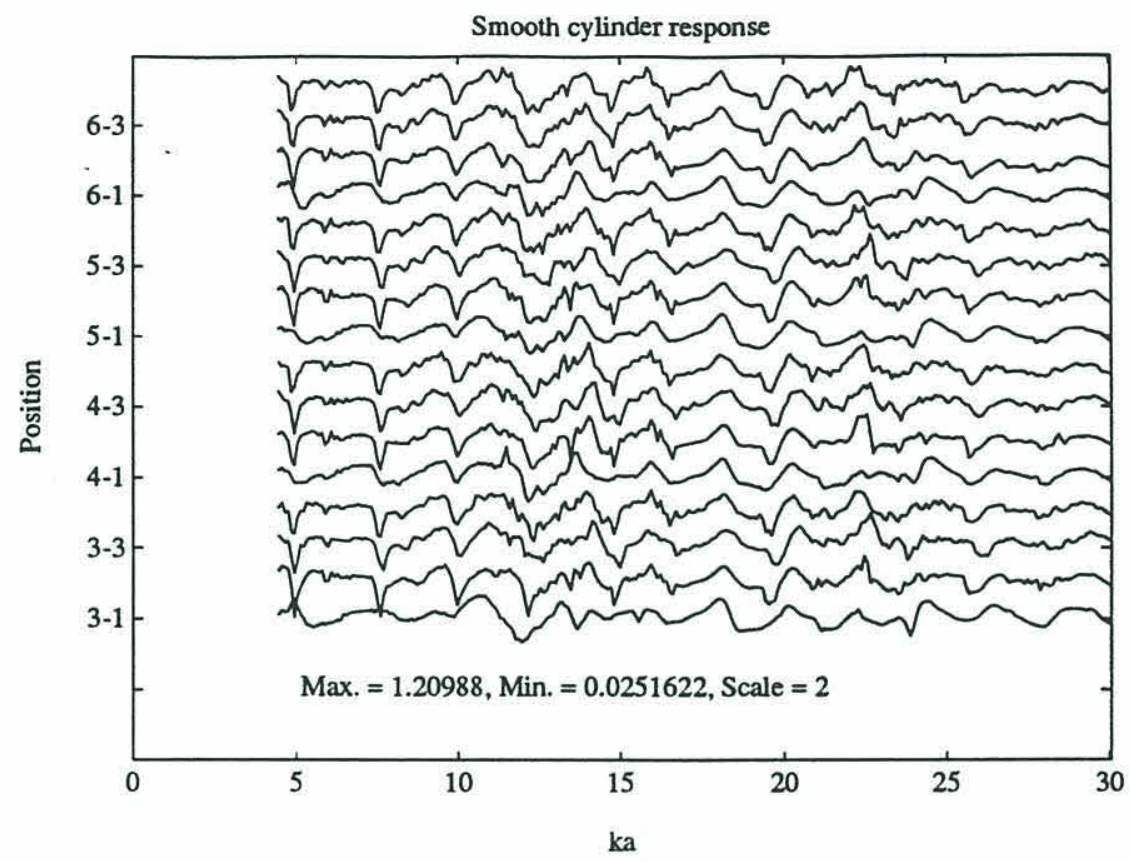

Figure 4-25: Normalized smooth cylinder frequency response measurements. Note missing nulls at $k a=5.0,8.0$, and 10.0 in positions $3-1,4-1,5-1$, and $6-1$ corresponding to measurements taken at a joint between subsections.

Using the smoothed calibration curve, we can now normalize all the smooth cylinder data and analyze the target frequency response. Any fine structure in the frequency response will be due to resonant modes in the target response since all this information has been "filtered" out of the calibration curve. Comparing the measured frequency response from all the smooth cylinder alignment measurements (Fig. 4-25) reveals the deep nulls at $k a=5.0,8.0$, and 10.0 are missing in the measurements $3-1,4-1,5-1$, and $6-1$. This occurred at the same transducer position, directly above the joint between subsections, during each run. Evidently the presense of the internal discontinuities in the joint destroys the modal interference which gives rise to these nulls. Measurements taken at position 2, just $3 \mathrm{~cm}$ away, show no effects, further indicating that this loss of the modal pattern is localized to scattering off of the joint itself.

Examination of the normalized response from the rough cylinder data (Fig. 4- 


\begin{tabular}{||c|r|r||}
\hline \hline & \multicolumn{1}{|c|}{$\begin{array}{c}\text { Smooth } \\
\text { Cylinder }\end{array}$} & $\begin{array}{c}\text { Rough } \\
\text { Cylinder }\end{array}$ \\
\hline \hline$\Delta k a$ & 0.01 & 0.1 \\
\hline$a_{o}$ & $0.456311 \mathrm{~cm}$ & $0.456311 \mathrm{~cm}$ \\
\hline$R$ & $24 \mathrm{~cm}$ & $24 \mathrm{~cm}$ \\
\hline$c$ & $1477 \mathrm{~m} / \mathrm{s}$ & $1477 \mathrm{~m} / \mathrm{s}$ \\
\hline$c_{s}$ & $3100 \mathrm{~m} / \mathrm{s}$ & $3100 \mathrm{~m} / \mathrm{s}$ \\
\hline$c_{l}$ & $5790 \mathrm{~m} / \mathrm{s}$ & $5790 \mathrm{~m} / \mathrm{s}$ \\
\hline$\rho_{1}$ & $7.9 \mathrm{~g} / \mathrm{cc}$ & $7.9 \mathrm{~g} / \mathrm{cc}$ \\
\hline$\Theta_{o}$ & $26^{\circ}$ & $26^{\circ}$ \\
\hline$\#$ Realizations & 1 & 50 \\
\hline \hline
\end{tabular}

Table 4.2: Parameters used for numerical simulations (material properties for Reference [45]).

26) reveals similar examples of loss of the nulls. These nulls are strong features that are independent of the roughness effects seen at higher $k a$ values. The exact position of the transducers relative to the joints in the rough target is not known for the rough cylinder but the loss of the nulls indicates that the transducers were insonifing a joint at these positions. The fact that this modal null loss occurs at similar positions during all three runs and that it repeats over 7 positions $(2 \mathrm{~cm}$ spacing) or a $14 \mathrm{~cm}$ spatial periodicity matches the $15 \mathrm{~cm}$ length of the subsections. Based on this, rough cylinder realizations $1-4,1-5,1-12,2-5,2-12,2-13,3-4,3-5$, $3-12$, and 3-13 were removed from the analysis due to interaction with a subsection joint. Similarly, smooth cylinder measurements $3-1,4-1,5-1$, and $6-1$ were also removed from the data set.

\subsection{Comparison with numerical results}

Using the 12 remaining backscatter measurements from the smooth cylinder, a comparison can be made with predictions from the numerical models in Chapter 3. Solutions for the scattered field were calculated using the measured laboratory parameters and published elastic properties of stainless steel (Table 4.2). Figure 4- 

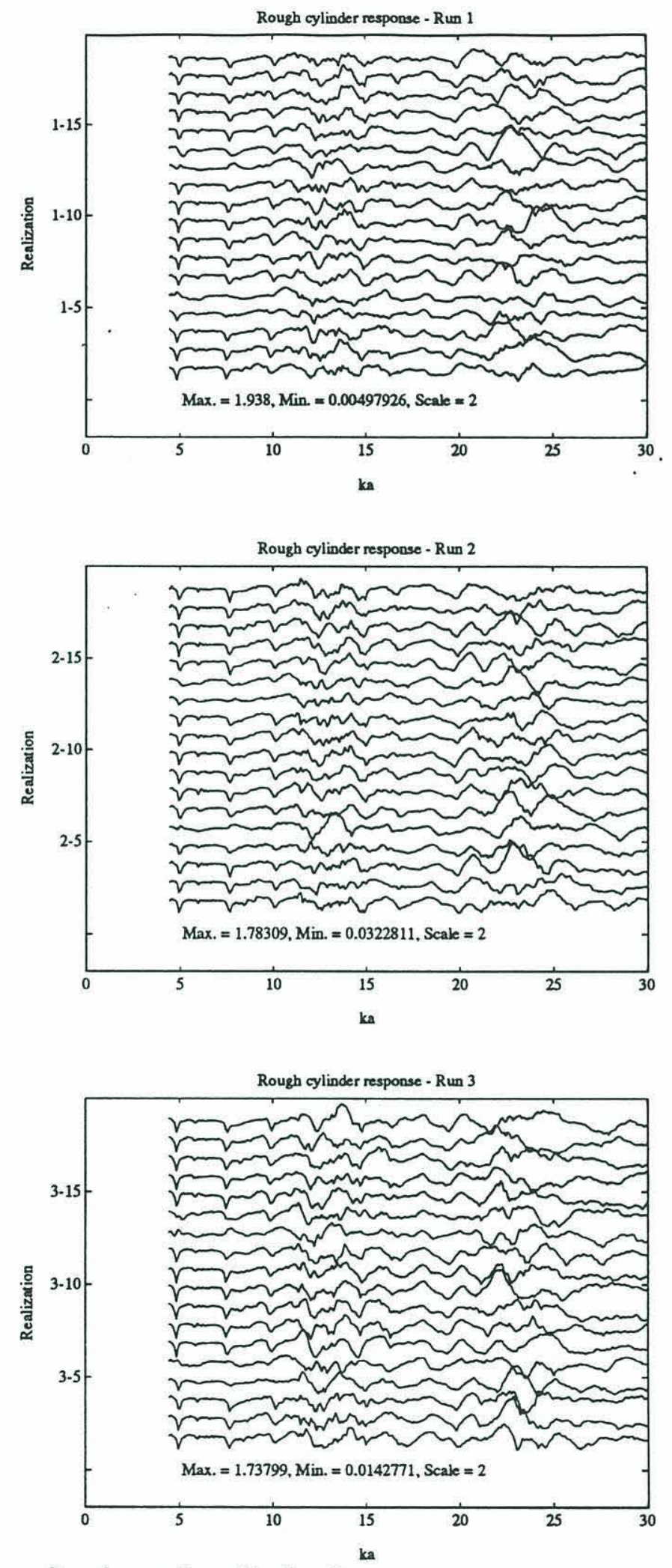

Figure 4-26: Normalized rough cylinder frequency response measurements for runs 1,2 , and 3 . 
27 compares the 12 smooth cylinder backscatter measurements to the model solution for a smooth elastic cylinder. The data has been normalized using the calibration curve in Fig. 4-23(b) to show the scattered form function $\left|f^{\infty}\right|$. The normalized data is only valid up to $k a=30$ which corresponds to the $1.5 \mathrm{MHz}$ upper frequency calibration limit discussed in Section 4.5. There is very good correlation between the predicted and measured field in both structure and levels over most of this frequency range. Figure 4-28 shows the ensemble average of the backscatter field over the 12 measurements Figures 4-29 and 4-30 show the variance of the smooth cylinder data and the correlation of the variance to the SNR during the measurements.

Figure 4-31 compares the scatter of the 44 rough cylinder backscatter measurements with the upper and lower bounds from a Monte Carlo simulation. The numerical model was run using the actual design surface profile of the target and the parameters listed in Table 4.2. Fifty realizations were calculated simulating moving the origin (and hence the source and receiver points) $2 \mathrm{~cm}$ down the $z$ axis between realizations. Figure 4-32 shows the relative variance, $\sigma_{|f \infty|}^{2} /\left\langle\left|f^{\infty}\right|^{2}\right\rangle$ of the backscatter data verses the values from the numerical model. As in the smooth cylinder data, there is a correlation between the relative variance and the SNR during the measurements (Fig. 4-33). Smoothing the relative variance curves using a 21 point running average weighted by the SNR value at each frequency point (Fig. 4-34) reveals the trend in the data as $k a$ and $k \sigma_{s}$ increase (the numerical data was also smoothed using the same relative weighting)

Even with the small sample size of 44 , the distribution of $\left|f^{\infty}\right|$ at most frequencies is well described by the Ricean PDF as predicted (Fig. 4-35). Here the shape parameter $\gamma$ has been calculated from the measured distribution of $\left|f^{\infty}\right|$ using Eq. (3.7) for low relative fluctuations and the least squares method described in Section 3.3 for high relative fluctuations. However, at frequencies near nulls (Fig. 4-36), the echo PDFs have a bimodal structure and skew; the Ricean PDF no longer describes the distribution in the data. Calculating $\gamma$ from the measured data and simulated 

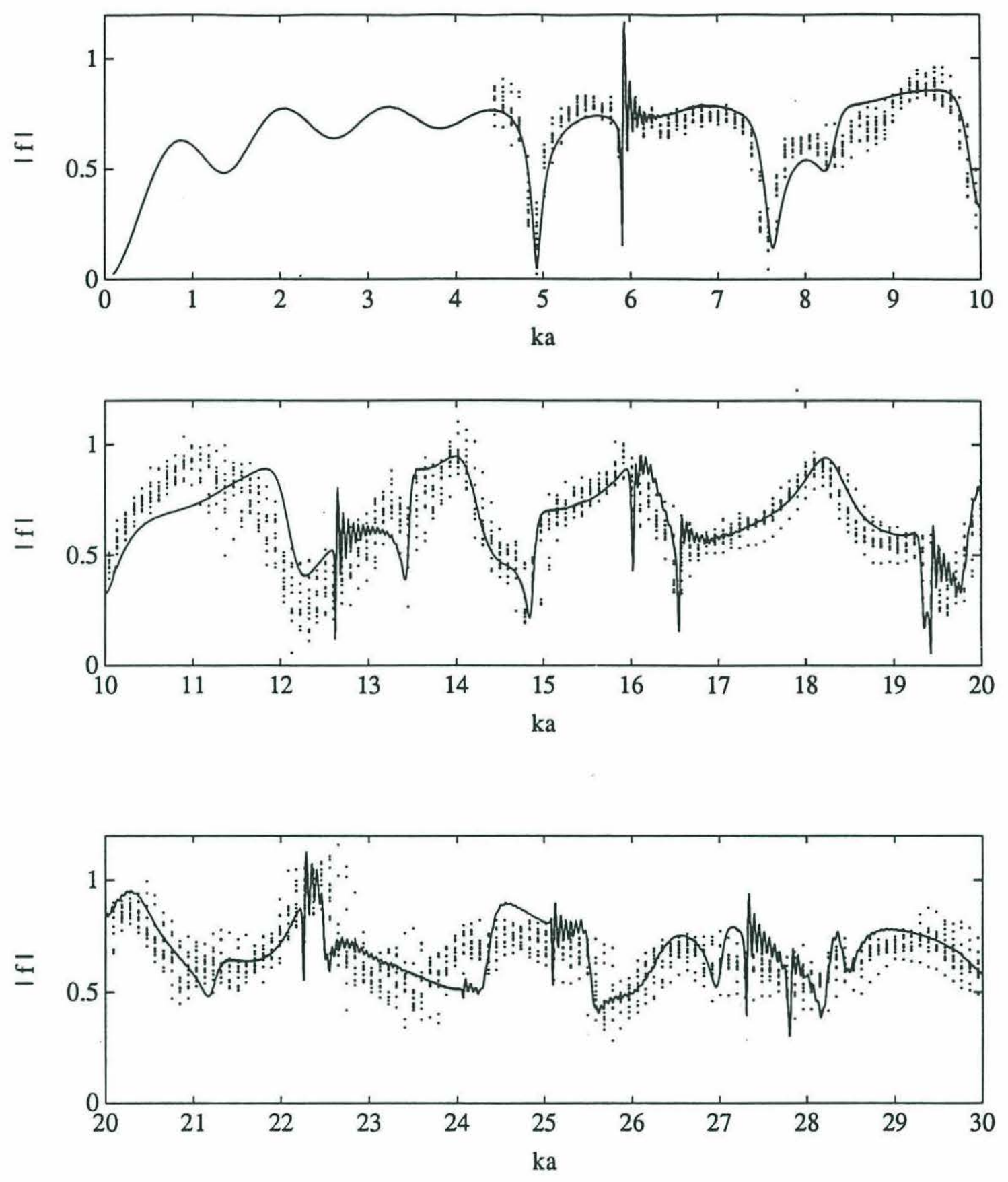

Figure 4-27: Measured backscattered (.) field from a smooth "infinite"-length stainless steel cylinder vs predicted field (-) from numerical simulation. Scatter plot formed from measurements taken at 12 different positions along the cylinder's $z$ axis. 

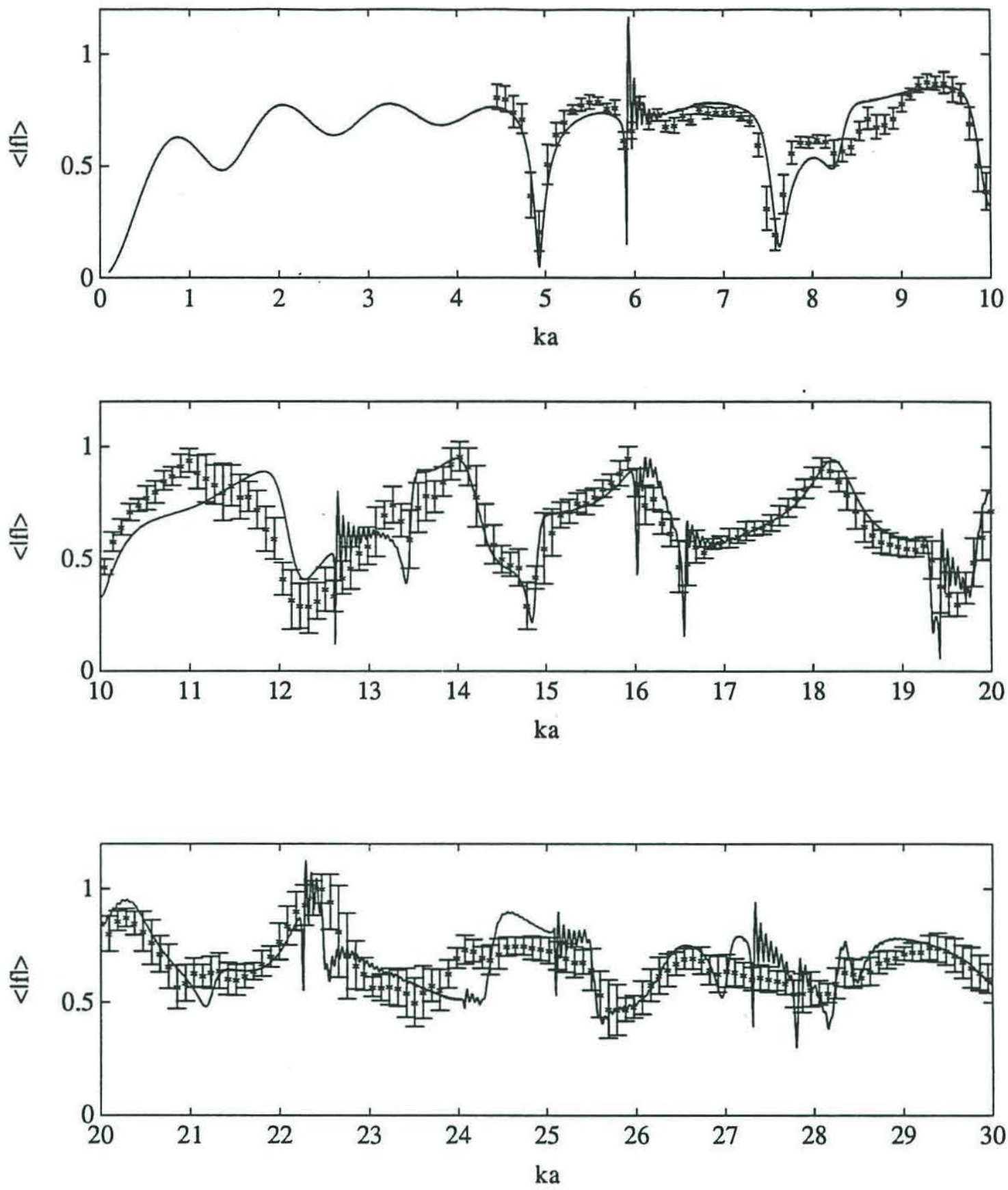

Figure 4-28: Mean of measured backscattered field from smooth "infinite"-length cylinder $\left({ }^{*}\right)$ vs predicted field (-). Mean measured field is an ensemble average of the 12 measurements in Figure 4-27. Errorbars indicate \pm 1 standard deviation, $\sigma_{|f \infty|}$, from the 12 measurements at each frequency. 

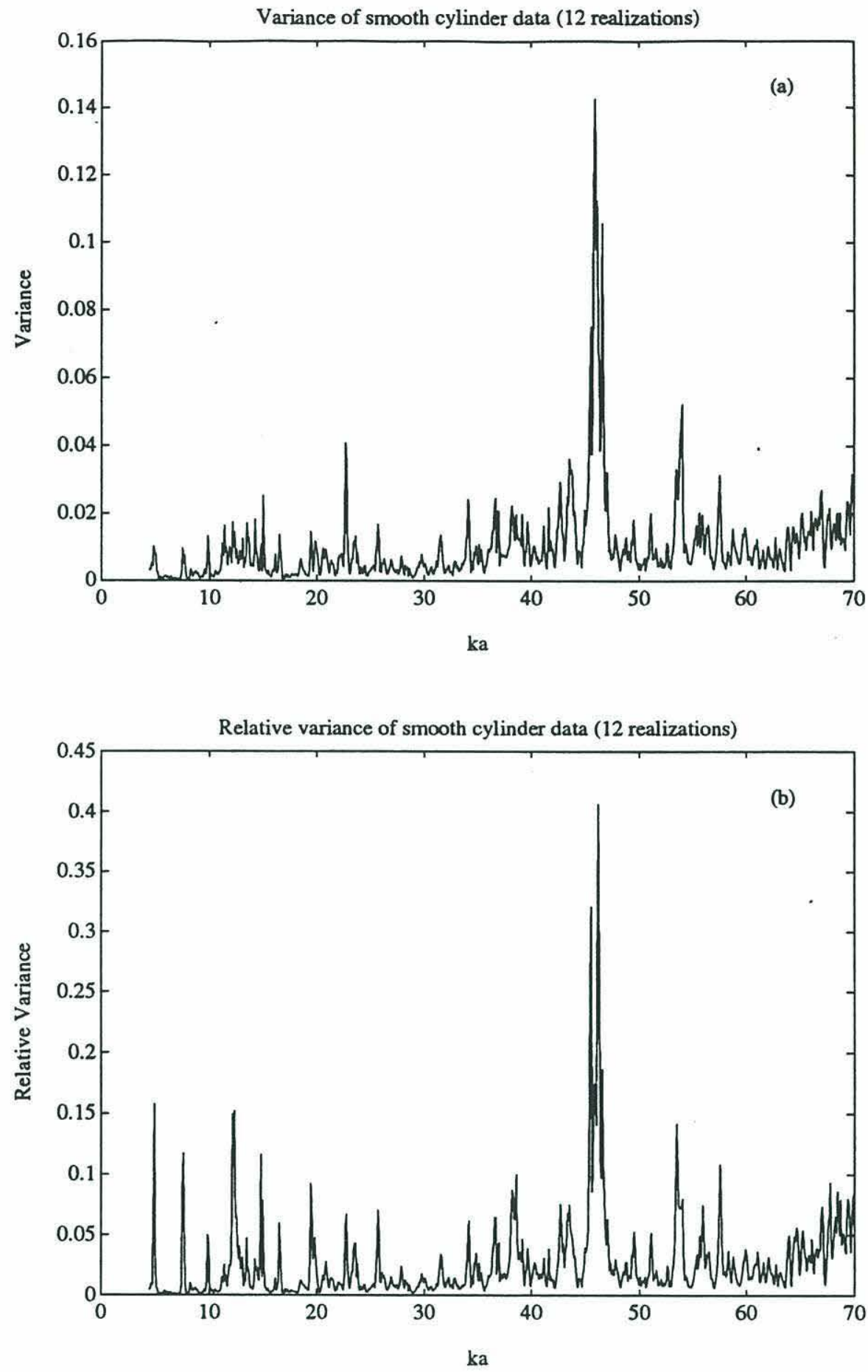

Figure 4-29: (a) Variance $\sigma_{|f \infty|}^{2}$ and (b) relative variance $\sigma_{|f \infty|}^{2} /\left\langle\left|f^{\infty}\right|\right\rangle^{2}$ of smooth cylinder backscatter data over the 12 realizations. 


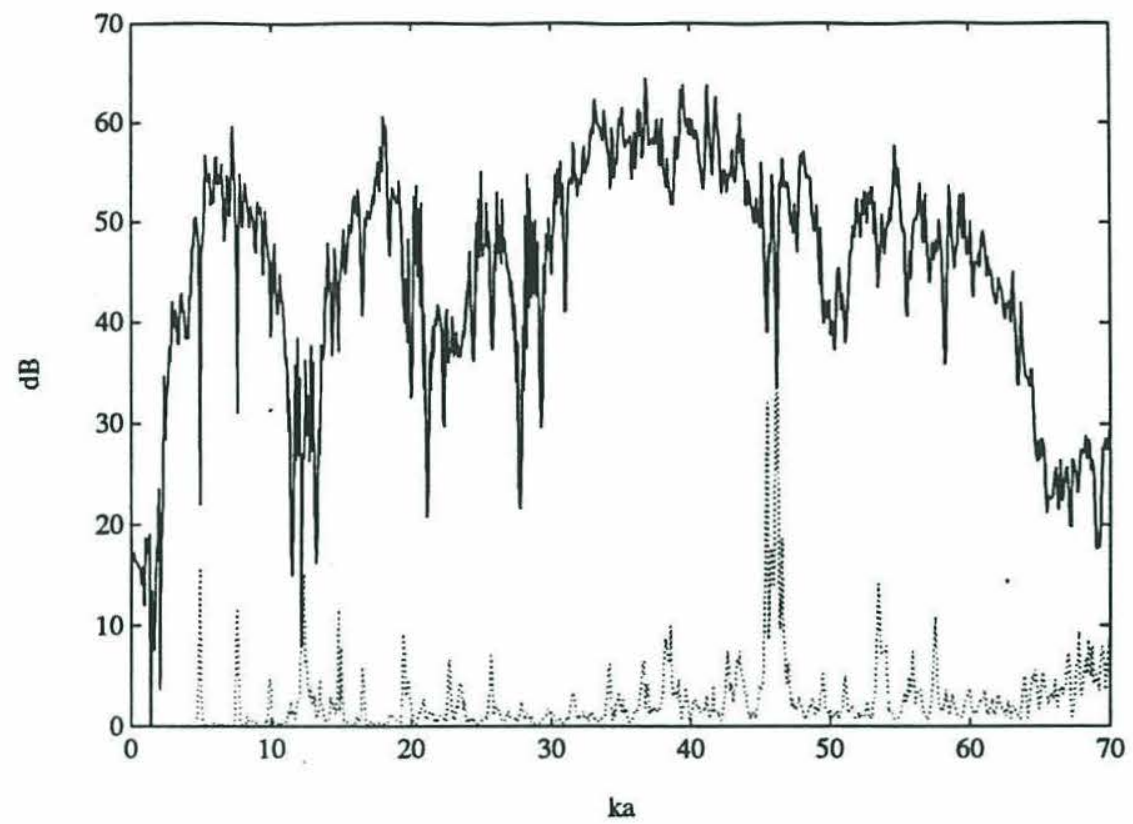

Figure 4-30: Sample SNR level (-) for smooth cylinder measurements overlaid with the relative variance $(\cdot)$ from Figure 4-29(b).

realizations (Fig. 4-37) for all $k a$ shows the excellent correlation between the data and the model predictions.

Finally, comparing the mean field of the rough cylinder data to the smooth cylinder data and their numerical models (Fig. 4-38) seems to show the decrease in the rough cylinder mean field level as predicted by the theory and the numerical simulations in Chapter 3. Only the frequency band corresponding to the best agreement between the smooth cylinder data and the analytic solution is shown. 

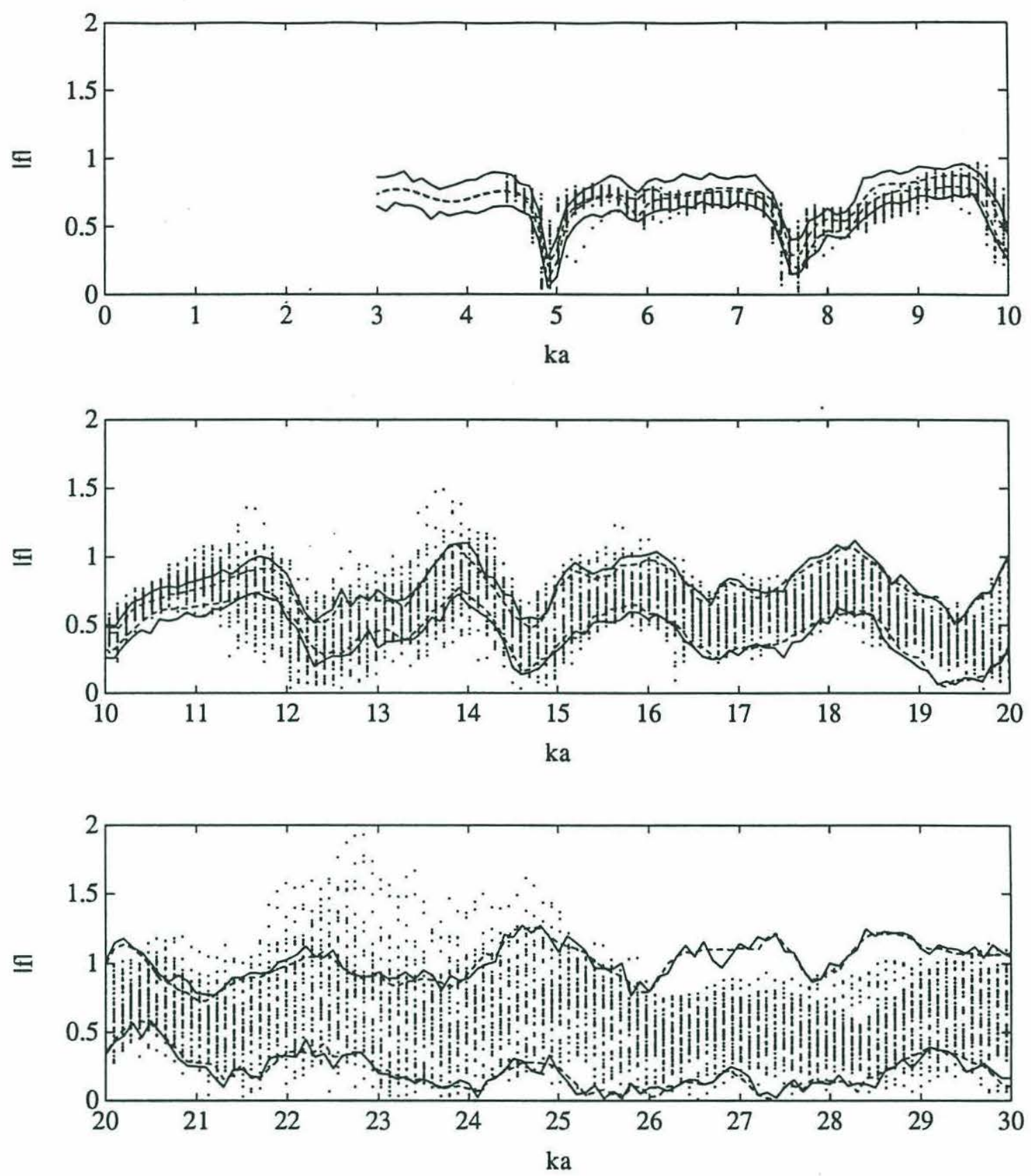

Figure 4-31: Measured backscattered field $(\cdot)$ from a randomly rough "infinite"-length stainless steel cylinder vs predicted minimum and maximum from Monte Carlo simulations with additive noise (-) and without additive noise (- -). Scatter plot formed from measurements of 44 realizations along the target axis and the numerical simulation is based on $\mathbf{5 0}$ simulated realizations using the profile of the target. Additive Gaussian white noise has been included in the Monte Carlo simulations to model the system related noise observed in the smooth cylinder data. 


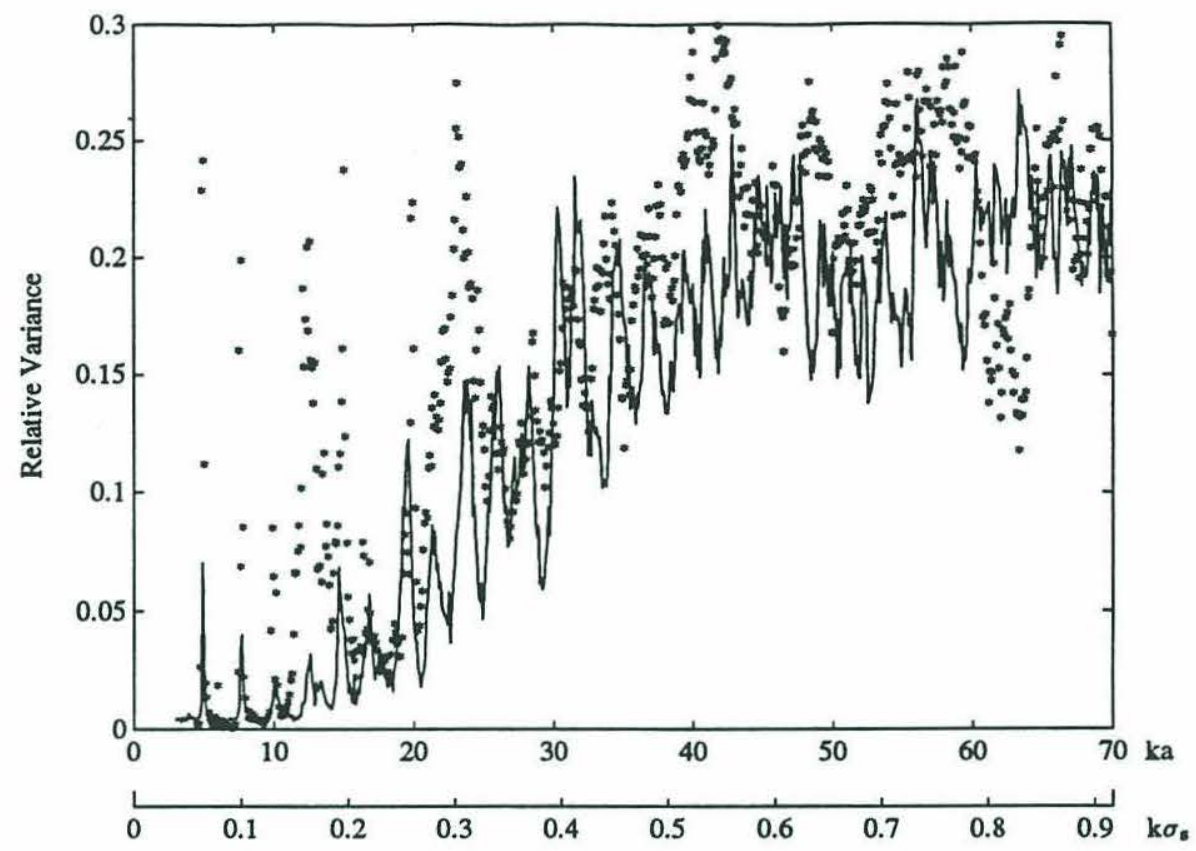

Figure 4-32: Relative variance $\sigma_{|f \infty|}^{2} /\left\langle\left|f^{\infty}\right|^{2}\right\rangle$ of rough cylinder backscatter data over the 44 realizations $\left({ }^{*}\right)$ vs numerical simulation including additive Gaussian white noise (一).

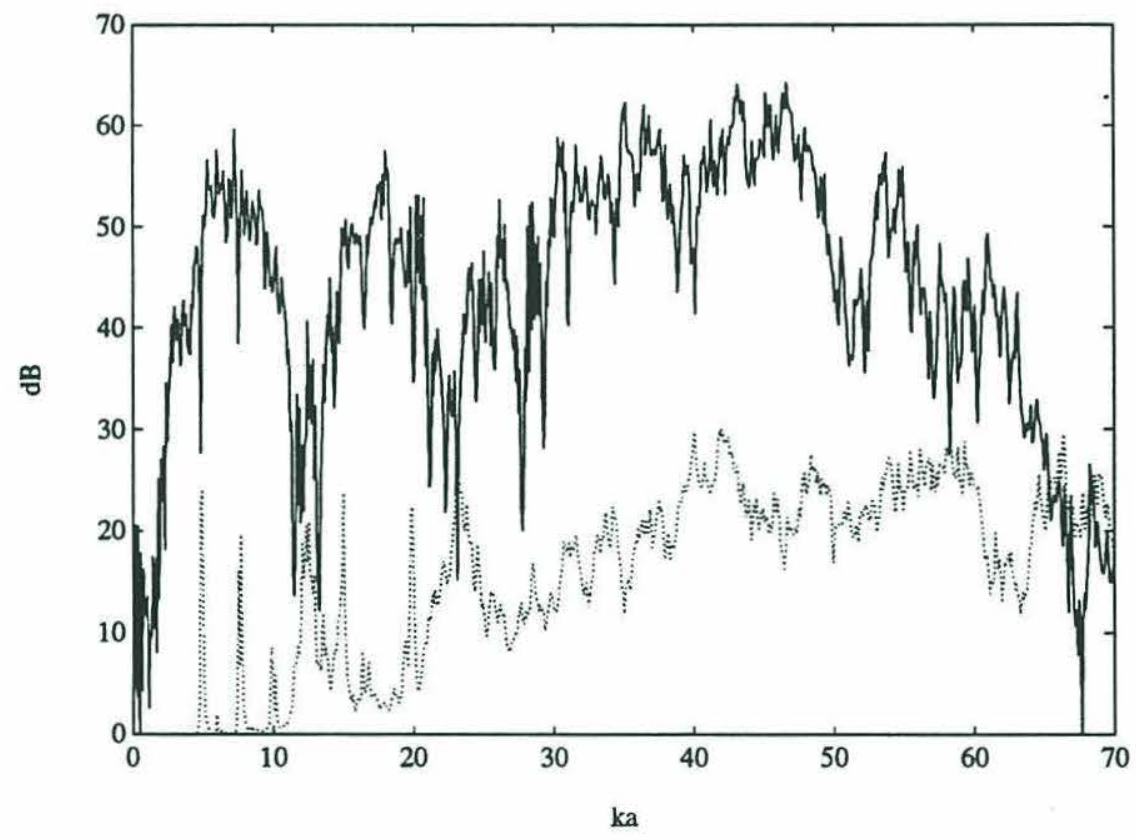

Figure 4-33: Sample SNR level (-) for rough cylinder measurements overlaid with

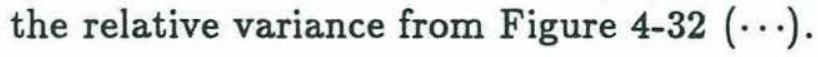




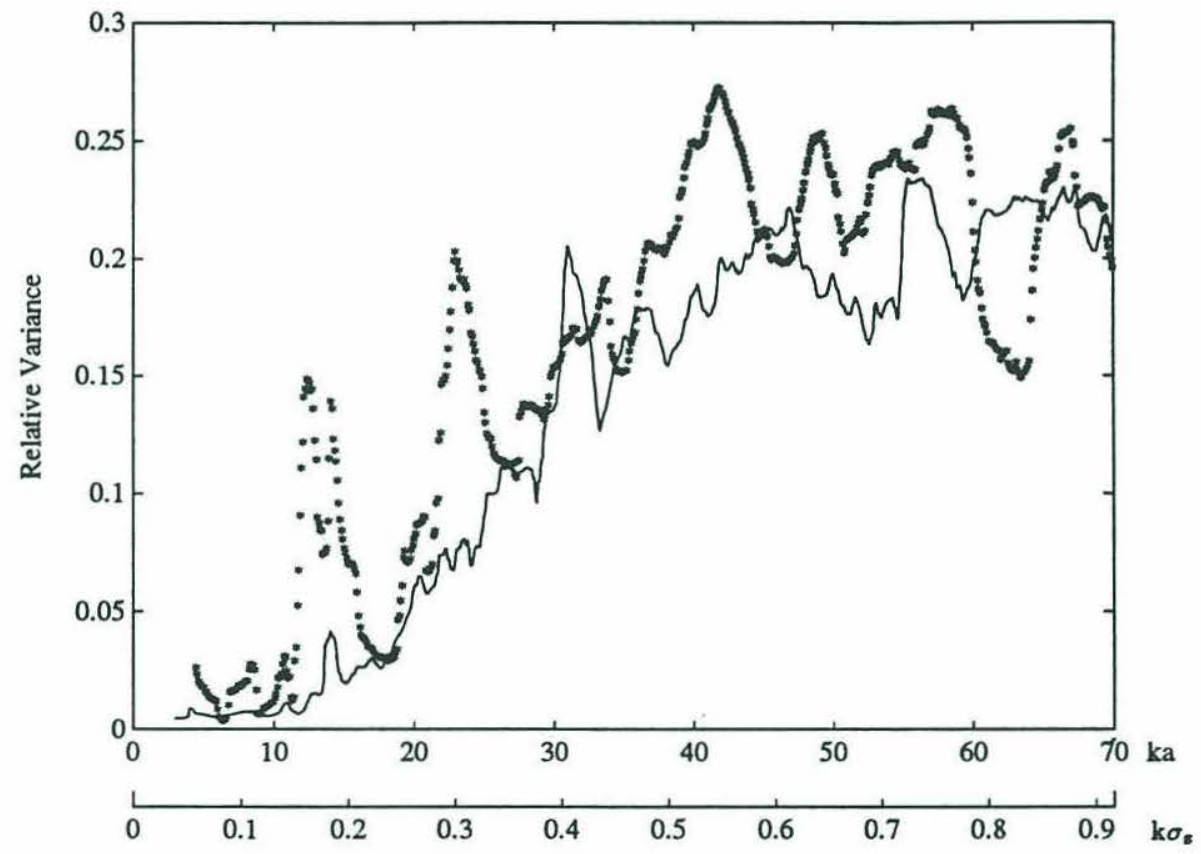

Figure 4-34: Relative variance $\sigma_{\mid f \infty \infty}^{2} /\left\langle\left|f^{\infty}\right|\right\rangle^{2}$ of rough cylinder backscatter data $\left({ }^{*}\right)$ vs the numerical prediction (-) after smoothing using a 21 point weighted running average. Simulation includes additive Gaussian white noise. 

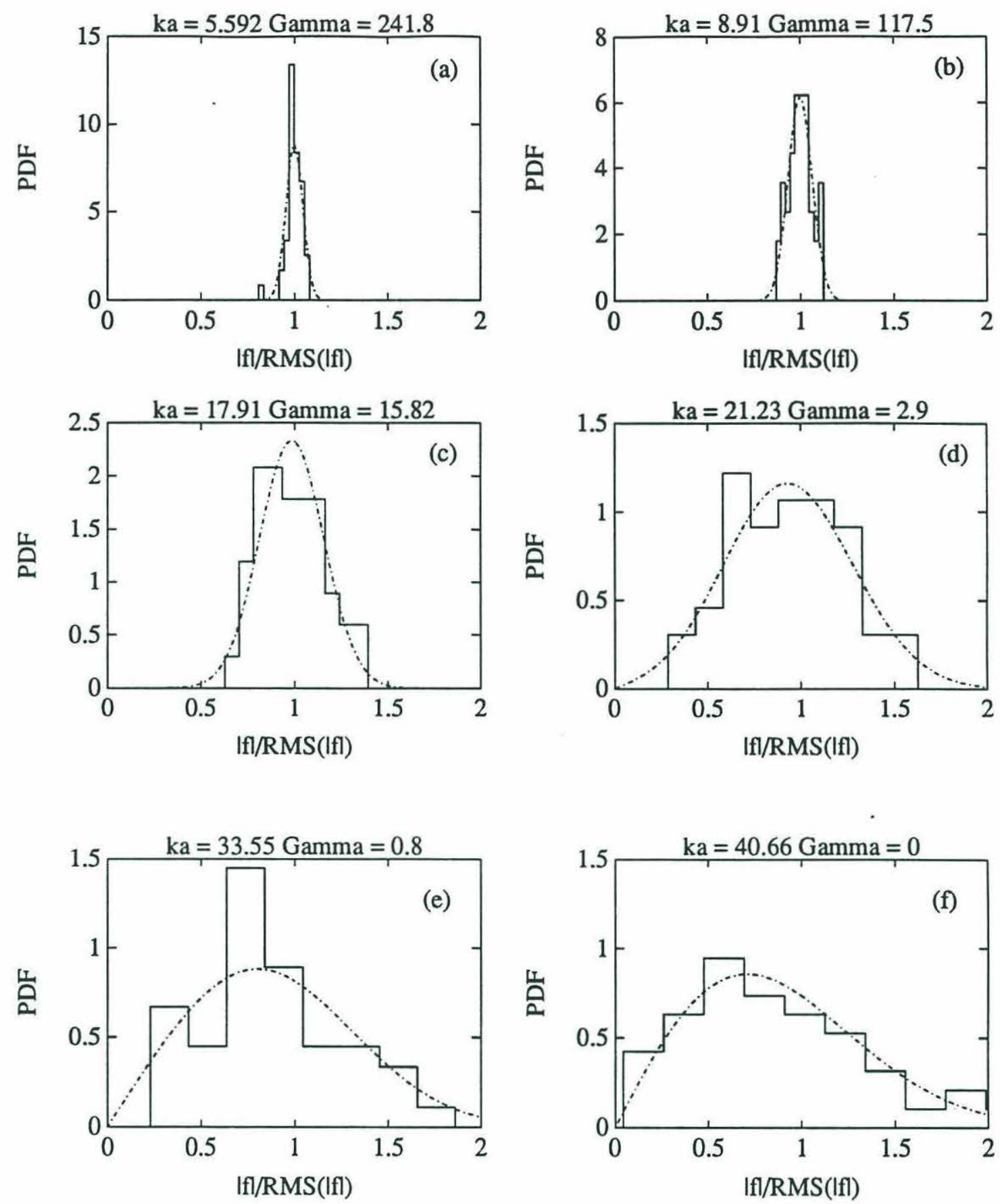

Figure 4-35: Histogram of measured rough cylinder backscatter data for various $k a$ values overlaid with Rice PDF using measured $\gamma(-\cdot-)$. These $k a$ values were chosen away from modal nulls. $k \sigma_{s}=0.0733,0.117,0.235,0.278,0.440,0.533$ for plots (a) - (f) respectively. 

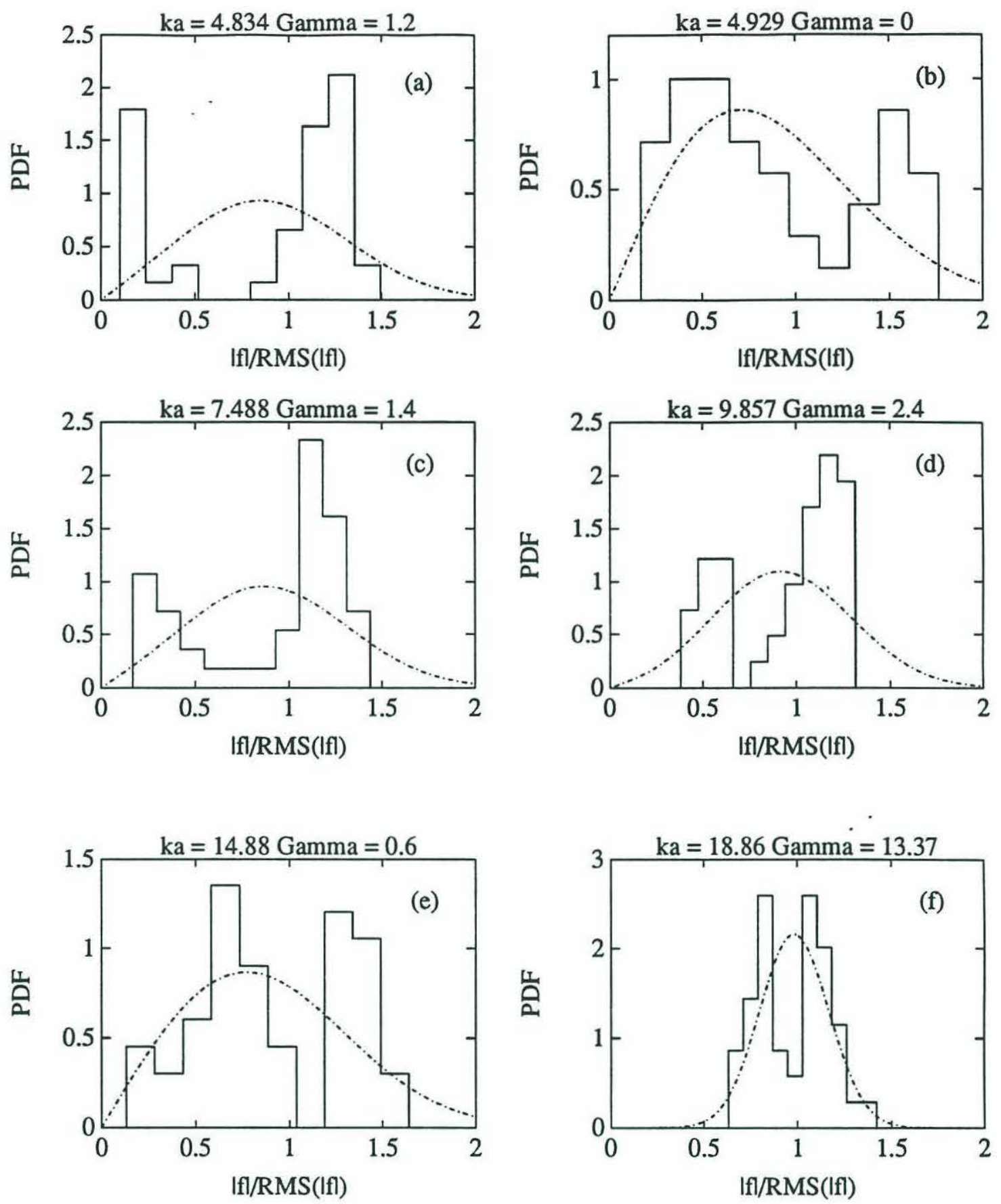

Figure 4-36: Histogram of measured rough cylinder backscatter data for various $k a$ values overlaid with Rice PDF using measured $\gamma(---)$. These $k a$ values were chosen at modal nulls. 

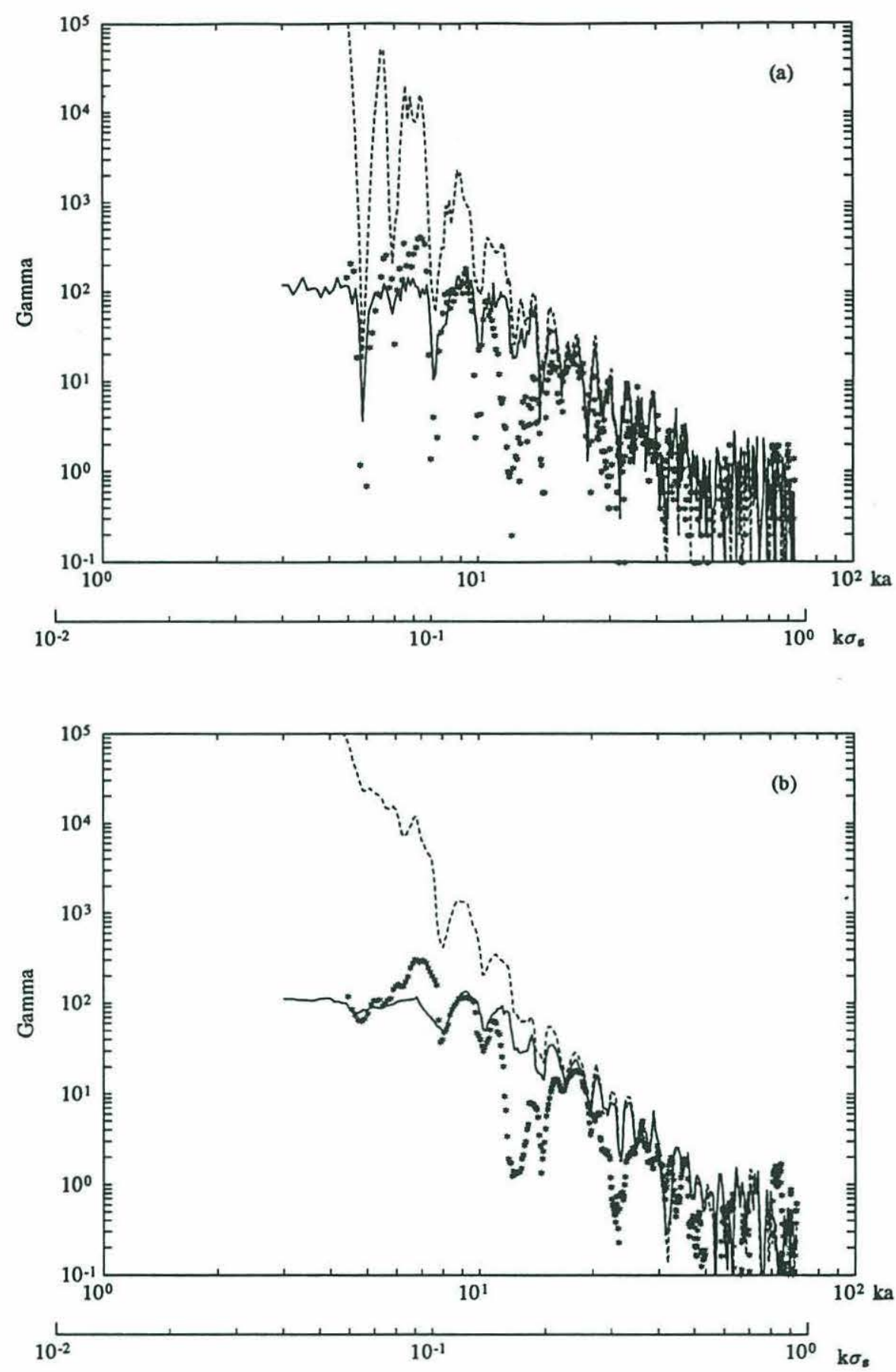

Figure 4-37: (a) Rice PDF shape parameter , $\gamma$, vs $k a$ and $k \sigma_{s}$. Rough cylinder backscatter data $\left({ }^{*}\right)$ is compared with an ideal or noiseless numeric simulation of 50 realizations (- - -) and numeric simulation with additive Gaussian white noise (-).(b) Same data after smoothing using 11 point weighted running average. The standard deviation of the additive noise was matched to the observed standard deviation "floor" in the smooth cylinder data $\left(\sigma_{|f \infty|}=0.05\right)$ 


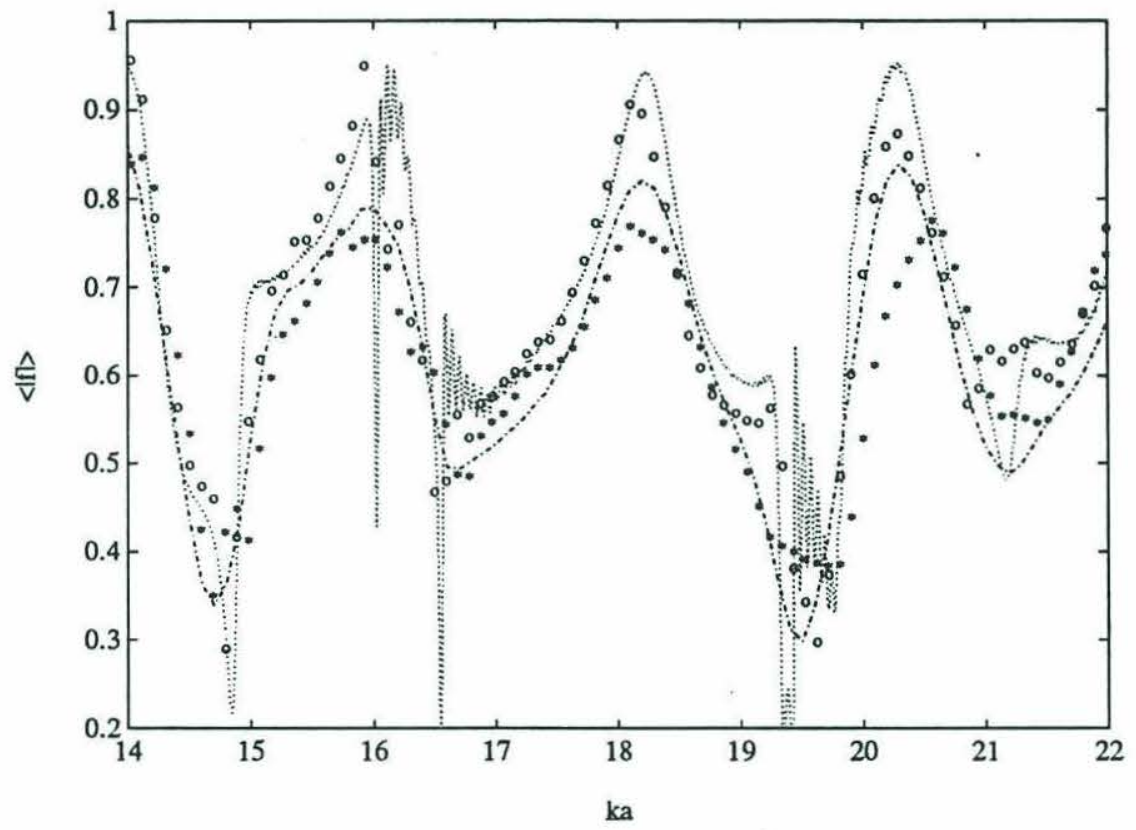

Figure 4-38: Mean of measured backscattered field from a randomly rough "infinite"-length cylinder $\left(^{*}\right)$ vs predicted mean field (---) overlaid with measured smooth cylinder mean field $(\circ)$ and smooth cylinder solution $(\cdots)$ from Fig. 4-28. The rough cylinder measured mean field $\left(^{*}\right)$ is an ensemble average of the 44 measurements in Fig. 4-31. 


\section{Chapter 5}

\section{Discussion of Results}

The backscatter measurements taken with the smooth cylinder target provide a baseline for comparison between the two data sets. Since both the smooth and rough targets were constructed from the same material and measurement procedures were identical, differences in the data sets are related to the surface roughness.

Figures 4-27 and 4-28 show that the smooth cylinder data follows the general trend and level of the predicted solution, but in many places, there is a mismatch in the modal structure between the two curves. We believe that this is due to imprecisions in the specification of the elastic properties of the target. No attempt was made to measure the actual wave velocities in the target and published values were used for the numerical solutions. Kaye and Laby [49] note that factors such as texture, cold work, stress, hardening, tempering, and aging can cause significant changes in the sound velocities in metals. As illustrated in Table 5.1, there are large variations in the published properties for stainless steel. The parameters published

\begin{tabular}{||c|r|r|r||}
\hline \hline Source & $c_{s} \mathrm{~m} / \mathrm{s}$ & $c_{l} \mathrm{~m} / \mathrm{s}$ & $\rho \mathrm{g} / \mathrm{cm}^{3}$ \\
\hline \hline AIP Handbook [45] & 3100 & 5790 & 7.9 \\
\hline Neubauer [35] & 3106 & 5594 & 7.9 \\
\hline Kaye and Laby [49] & 3297 & 5980 & 7.8 \\
\hline \hline
\end{tabular}

Table 5.1: Material properties for stainless steel from various sources 
in the AIP tables were used because they provided the closest match with the observed modal structure in the data. Different values significantly affected the modal structure and the relative position of the peaks and nulls in the numerical solution. In Fig. 4-28, the smooth cylinder data most closely match the modal structure of the numerical solution for $14 \leq k a \leq 22$ so this is the region where we can expect the best conditions for objective comparison of the rough cylinder data with the theoretical predictions.

The smooth cylinder data also contain some small fluctuations between realizations (Figs. 4-27 and 4-29). These deviations between individual measurements reflect the accumulation of systematic errors inherent in any experiment; alignment errors, measurement errors, calibration errors (as already discussed), signal to noise ratio, bubbles and suspended particles, nonuniformities in the cylinder, surface microroughness, and anisotropies in the material properties all contribute to the variance, $\sigma_{|f \infty|}^{2}$, in the data between smooth cylinder measurements. Since the calibration of the data for $k a>30$ could not be established, we analyzed the relative variance (variance divided by the mean square); this self-normalizes the data for all $k a$. Figure 4-29 shows the variance and the relative variance for the smooth cylinder data has a general "floor" which gradually increases with $k a$ (as acoustic wavelength decreases). There is a large localized peak in the variance at $k a=46$ and smaller spikes throughout the data. Comparing the normalized variance with a scaled sample SNR curve for the experiment (Fig. 4-30) shows that the large peak at $k a=46$ correlates with a dip in the SNR and that the smaller spike at $k a=12$ also correlates with a sharp dip in the SNR. The variance shows a sharp rise above $k a=65$ as the SNR falls off at the upper limit of the transducer response.

The rough cylinder time series (Figs. 4-19 - 4-21) illustrate the complicated nature of the rough surface scattering. There are now multiple "specular" arrivals from different facets of the surface in the main beam causing a temporal spread in the "specular" arrival time. Likewise, each realization contains an ensemble of ap- 
proximately independent radii from each differential element along the cylinder axis causing a temporal spread in the Rayleigh wave arrival times and large fluctuations in its relative amplitude.

The scatter in the normalized rough cylinder response (Fig. 4-31) is well described by the numerical simulations. Comparisons using several different random profiles showed the closest correlation with the data when the actual target profile was used in the simulation and the number of simulated realizations was the same order as the data set. The data shows the same variance "floor" at low $k a$ as seen in the smooth cylinder data, but above $k a=10$, there is a clear increase in the scatter in the measurements due to the rough surface profile which is well predicted by the upper and lower bounds of the numerical model. There are also localized increases in the data fluctuations around $k a=12$ and $k a=23$ which are not described by the numerical bounds. The relative variance of the data (Fig. 4-32) shows an overall increase in variance due to the rough surface effects. The data follow the general trend of the numerical prediction and clearly exhibit a transition point and plateau at $k \sigma_{s}=0.5$. The large spikes in the data variance at low $k a$ are due to the very low signal levels in the resonant nulls. Comparing the relative variance to the SNR levels for the rough cylinder experiment (Fig. 4-33), shows the "noise" peaks at $k a=12$ and 22 correlate with large drops in the signal to noise ratio at those frequencies. This explains the increased scatter in the data noted in Fig 4-31 at those frequencies. Smoothing the relative variance plot using a weighted running average based on SNR (Fig. 4-34), highlights the good agreement between the data trend and the numerical prediction. Again we see that as $k \sigma_{s}$ increases and the surface roughness has a greater effect in the scattering process, the variance in the backscatter increases and reaches a saturation level at $k \sigma_{s}=0.5$.

Theoretically, the variance goes to zero as $k \sigma_{s}$ goes to zero and Rice PDF shape parameter $\gamma$ becomes infinite. However, as we have already seen from the smooth cylinder data, the experimental data have a finite noise "floor" even at low ka. This 
system related noise "floor" is modeled as zero mean additive Gaussian white noise and included in the numerical simulations. Figure 4-37 illustrates the improved fit between the data and the simulations when this additive noise is included. The standard deviation of the additive noise was matched to the observed standard deviation in the smooth cylinder data. Now we can identify three distinct regions in the data:

1. A low roughness region $\left(k \sigma_{s} \leq 0.20\right)$ dominated by system noise where $\gamma$ follows the predicted structure but the level rolls off.

2. A transition from low to high roughness $\left(0.20 \leq k \sigma_{s} \leq 0.50\right)$ where $\gamma \propto k^{-4}$.

3. A high roughness region $\left(k \sigma_{s}>0.5\right)$ where $\gamma$ levels off near 0 .

The sharp spikes in Fig. 4-37 are correlated with the modal nulls in the target response. These local maxima in fluctuation (local minima in $\gamma$ ) arise when the value of $k a$ matches or nearly matches a modal null region. Small changes in the local $k a$ will move the response in or out of the null region and cause large changes in scattered amplitude. This increases the variance over the ensemble of realizations and causes the pattern of peaks in Fig. 4-32 and dips in Fig. 4-37. This phenomenon is evident in both the numerical model and the data.

Comparing the mean value of the rough cylinder data with the mean value of the smooth cylinder data and comparing both with the numerical predictions (Fig. 4-38), shows a roughness-induced decrease in the mean field as predicted by the numerical simulations. The data and models show excellent agreement at least up to $k a=20$ and while the correlation breaks down (due to the unresolved calibration issue) above $k a=20$ the data show the same relative trend and the same relative decrease as the theoretical model.

Summarizing the major points:

- The specular and Rayleigh wave arrival times are "smeared" out due to the 
perturbed acoustic path lengths caused by the random radius perturbations (Figs. 4-19-4-21).

- Even for a small sample size (44 realizations), the Rice PDF characterizes the echo distributions for most values of $k a$ away from sharp nulls (Fig. 4-35 and $4-36)$.

- The bounds of scatter in the backscatter data are reasonably well described by the maximum and minimum of the numerical model for an equivalent number of realizations using the actual target profile (Fig. 4-31).

- The variance of the data is bounded by a noise "floor" and correlated with the system-related variations for all measurements (Figs. 4-29, 4-30, 4-32, and $4-34)$.

- Once the additive white noise is included in the numerical simulations to account for system effects, there is a greatly improved correlation with the observed change in $\gamma$ as $k \sigma_{s}$ increases (Fig. 4-37).

- The predicted turning point between low and high roughness regions is observed at $k \sigma_{s}=0.5$ (Figs. 4-32, 4-34, and 4-37).

- The mean backscattered field from the rough cylinder follows both the structure and level of the theory (at least to the extent that the calibration and material properties are sufficiently known) and shows the predicted decrease in the mean level (Fig. 4-38). 


\section{Chapter 6}

\section{Conclusions}

The data presented show the effects of small scale surface roughness on the scattering from a body of curvature. By randomizing the radius and including realistic source terms in the deformed cylinder formulation, we have been able to accurately model the observed scattering phenomena from a randomly rough elastic cylinder. We have shown the importance of careful description of the target profile and of precise alignment in order to quantitatively predict and measure roughness-related effects in the scattered field. The summary of major results listed in Chapter 5 details many of the aspects of rough body scattering that have been predicted but not previously observed due to the lack of controlled data sets.

This data set represents one set of surface statistics for one value of surface roughness. More work is required over a broad range of surface roughness scales to verify the results obtained here and investigate such features as the decrease in the mean scattered field as $\sigma_{s} / a$ increases. Perfectly smooth objects do not exist even in the laboratory, and much more work is required on this general problem if we wish to truly understand the scattering process.

While there are similarities to rough planar interface scattering which has been heavily studied, we have seen the circumferential waves arising from the curvature of volumetric bodies provide a source of new and unexamined phenomena. The 
deformed cylinder formulation has been a powerful tool in predicting the roughnessrelated effects in both the mean and the fluctuations of the scattered field, but it is limited to elongated shapes. More general formulations are needed to describe rough spheres and other shapes as well as to consider truly two-dimensional surface roughness.

Whereas we have focused on the "forward" problem of predicting then measuring scattering from a known rough surface, future work could investigate the inverse problem of extracting information from the scattered field in order to infer the profile of an unknown target surface. Just as early work on rough interface scattering proved essential to the design and development of a wide variety of currently employed sonar systems and techniques, research into general rough body scattering will provide the necessary foundation for improvements in the "state of the art". 


\section{Bibliography}

[1] C. Eckart, "The scattering of sound from the sea surface," J. Acoust. Soc. Am., 25, 566-570 (1953).

[2] C.S. Clay and H. Medwin, Acoustical Oceanography: Principles and Applications (Wiley-Interscience, New York, 1977).

[3] T.K. Stanton, K.C. Jezek, and A.J. Gow, "Acoustical reflection and scattering from the underside of laboratory grown sea ice: Measurements and predictions," J. Acoust. Soc. Am., 80, 1486-1494 (1986).

[4] T.K. Stanton, "Sonar estimates of seafloor microroughness," J. Acoust. Soc. Am., 75, 809-818 (1984).

[5] J.B. Mehl, "Acoustic resonance frequencies of deformed spherical resonators.II," J. Acoust. Soc. Am., 79, 278-285 (1986).

[6] Y.P. Lysanov and Y.Y. Popov, "Propagation of creeping waves around a statistically rough sphere," Sov. Phys. Acoust., 35, 502-505 (1989).

[7] Y.Y. Popov, "Sound scattering by a statistically corrugated cylindrical surface," Sov. Phys. Acoust., 31, 509-511 (1985).

[8] Y.Y. Popov, "Propagation of creeping waves around a statistically rough cylindrical surface," Sov. Phys. Acoust., 33, 189-192 (1987). 
[9] F.G. Bass and I.M. Fuks, Wave Scattering from Statistically Rough Surfaces, translated by C.B. Vesecky and J.F. Vesecky (Pergamon Press, Oxford, 1979).

[10] Y.M. Chen, "Diffraction by a smooth transparent object," J. Math. Phys., 5, 820-832 (1964).

[11] P. Barber and C. Yeh, "Scattering of electromagnetic waves by arbitrarily shaped dielectric bodies," App. Opt., 14, 2864-2872 (1975).

[12] V.K. Varadan, V.V. Varadan, and Y.-H. Pao, "Multiple scattering of elastic waves by cylinders of arbitrarily cross section. I. SH waves," J. Acoust. Soc. Am., 63, 1310-1319 (1978).

[13] J.C. Erdmann and R.I. Gellert, "Speckle field of curved, rotating surfaces of Gaussian roughness illuminated by a laser light spot,” J. Opt. Soc. Am., 66, 1194-1204 (1976).

[14] T.K. Stanton, "Sound scattering by cylinders of finite length. I. Fluid cylinders,” J. Acoust. Soc. Am., 83, 55-63 (1988).

[15] T.K. Stanton, "Sound scattering by cylinders of finite length. II. Elastic cylinders," J. Acoust. Soc. Am., 83, 64-67 (1988).

[16] T.K. Stanton, "Sound scattering by cylinders of finite length. III. Deformed cylinders," J. Acoust. Soc. Am., 86, 691-705 (1989).

[17] T.K. Stanton, "Sound scattering by rough elongated elastic objects. I. Means of scattered field," J. Acoust. Soc. Am., 92, 1641-1664 (1992).

[18] T.K. Stanton and D. Chu, "Sound scattering by rough elongated elastic objects. II. Fluctuations of scattered field," J. Acoust. Soc. Am., 92, 1665-1678 (1992). 
[19] Lord Rayleigh, The Theory of Sound (Dover, New York, second edition, 1945 reissue) (1877).

[20] P.M. Morse and K.U. Ingard, Theoretical Acoustics (McGraw-Hill, New York, 1968).

[21] E. Skudrzyk, The Foundations of Acoustics (Springer-Verlag, New York, 1971).

[22] F.B. Hildebrand Advanced Calculus for Applications (Prentice-Hall, Englewood Cliffs, NJ, 1976), second edition.

[23] Handbook of Mathematical Functions, edited by M. Abramowitz and I.A. Stegun (Dover, New York, 1965).

[24] J.J. Faran, Jr., "Sound scattering by solid cylinders and spheres," J. Acoust. Soc. Am., 23, 405-418 (1951).

[25] A.E.H. Love, A Treatise on the Mathematical Theory of Elasticity, (Dover, New York, fourth edition, 1944 reissue) (1927).

[26] R.D. Doolittle, H. Überall, and P. Ugincius, "Sound scatter by elastic cylinders," J. Acoust. Soc. Am., 43, 1-14 (1968).

[27] D. Brill and H. Überall, "Acoustic waves transmitted through solid elastic cylinders," J. Acoust. Soc. Am., 50, 921-939 (1971).

[28] G.V. Frisk, J.W. Dickey, and H. Überall, "Surface wave modes on elastic cylinders," J. Acoust. Soc. Am., 58, 996-1008 (1975).

[29] J.W. Dickey, G.V. Frisk, and H. Überall, "Whispering Gallery wave modes on elastic cylinders," J. Acoust. Soc. Am., 59, 1339-1346 (1976).

[30] J.W. Dickey and H. Überall, "Acoustic high-frequency scattering by elastic cylinders," J. Acoust. Soc. Am., 66, 275-283 (1979). 
[31] K.L. Williams and P.L. Marston, "Axially focused (glory) scattering due to surface waves generated on spheres: Model and experimental confirmation using tungsten carbide spheres,” J. Acoust. Soc. Am., 78, 722-728 (1985).

[32] K.L. Williams and P.L. Marston, "Backscattering from an elastic sphere: Sommerfeld-Watson transformation and experimental confirmation," J. Acoust. Soc. Am., 78, 1093-1102 (1985).

[33] K.L. Williams and P.L. Marston, "Synthesis of backscattering from an elastic sphere using the Sommerfeld-Watson transformation and giving a Fabry-Perot analysis of resonances,” J. Acoust. Soc. Am., 79, 1702-1708 (1986).

[34] P.L. Marston, "GTD for backscattering from elastic spheres and cylinders in water and the coupling of surface waves with the acoustic field," J. Acoust. Soc. Am., 83, 25-37 (1988).

[35] Acoustic Reflection from Surfaces and Shapes, edited by W.G. Neubauer (Naval Research Laboratory, Washington D.C., 1986).

[36] D.T. DiPerna and T.K. Stanton, "Fresnel zone effects in the scattering of sound by cylinders of various lengths," J. Acoust. Soc. Am., 90, 3348-3355 (1991).

[37] A. Ishimaru, Wave Propagation and Scattering in Random Media, Volume I: Single Scattering and Transport Theory (Academic Press, New York, 1978).

[38] E. Hecht and A. Zajac, Optics (Addison-Wesley, Reading, MA, 1974).

[39] G.C. Gaunaurd, "Sonar cross sections of bodies partially insonified by finite sound beams," IEEE J. Ocean. Eng., 10, 213-230 (1985).

[40] I.B. Andreeva and V.G. Samovol'kin, "Sound scattering by elastic cylinders of finite length," Sov. Phys. Acoust., 22, 361-364 (1976). 
[41] D.L. Fried, "Statistics of the laser radar cross section of a randomly rough target," J. Opt. Soc. Am., 66, 1150-1160 (1976).

[42] S.O. Rice, "Mathematical analysis of random noise," in Selected Papers on Noise and Stochastic Processes, edited by N. Wax (Dover, New York, 1954), pages 133-294.

[43] G. Dahlquist and-A. Björck, Numerical Methods, translated by N. Anderson (Prentice-Hall, Englewood Cliffs, NJ, 1974).

[44] W.H. Press, B.P. Flannery, S.A. Teukolsky, and W.T. Vetterlin, Numerical Recipes in $C$ (Cambridge University Press, Cambridge, 1988).

[45] American Institute of Physics Handbook, edited by D.E. Gray (McGraw-Hill, New York, 1972), third edition.

[46] Modern Spectrum Analysis, edited by D.G. Childers (IEEE Press, New York, 1978).

[47] A.V. Oppenheim and R.W. Schafer, Discrete-Time Signal Processing (Prentice-Hall, Englewood Cliffs, NJ, 1989).

[48] T.K. Stanton, "Sound scattering by spherical and elongated shelled bodies," J. Acoust. Soc. Am., 88, 1619-1633 (1990).

[49] G.W.C. Kaye and T.H. Laby, Tables of Physical and Chemical Constants (Longman, New York, 1973). 